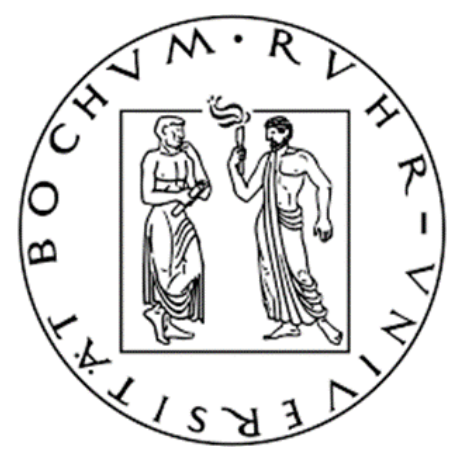

Ruhr-Universität Bochum

-Fakultät für Psychologie-

\title{
Manual or Automatic? Putting standardized EEG-Preprocessing to the Test with the Alpha-Asymmetries of Personality
}

Masterarbeit zur Erlangung des akademischen Grades
Master of Science (M.Sc.)

Vorgelegt von

Javier Eduardo Schneider Peñate Matrikelnummer: 108016258450 Javier.SchneiderPenate@rub.de

September 2021

Erstgutachter: Prof. Dr. Sebastian Ocklenburg

Zweitgutachterin: M.Sc. Dorothea Metzen 


\section{Table of Contents}

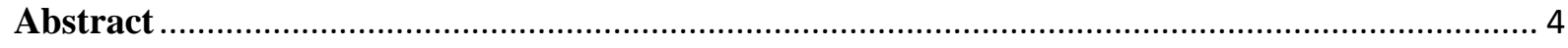

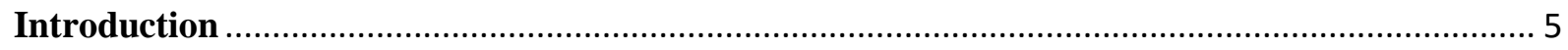

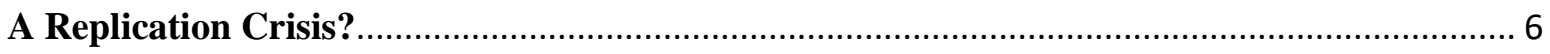

Current Scientific Practices: Flaws and Solutions ……....................................................... 7

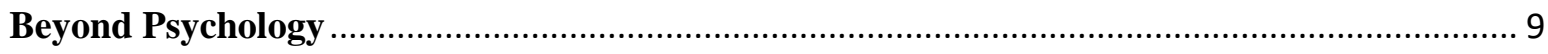

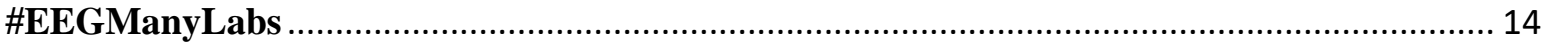

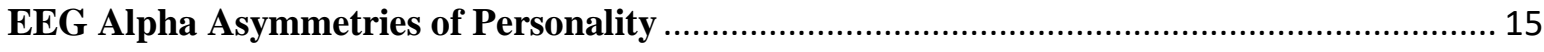

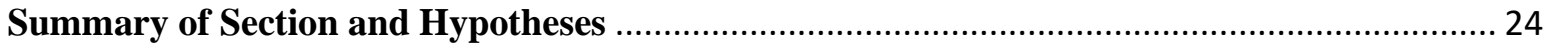

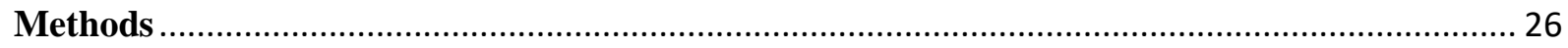

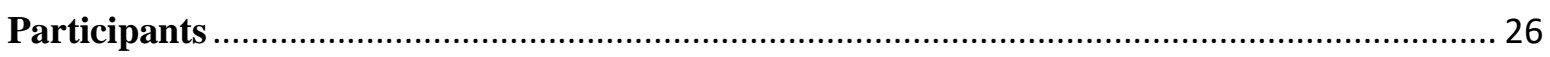

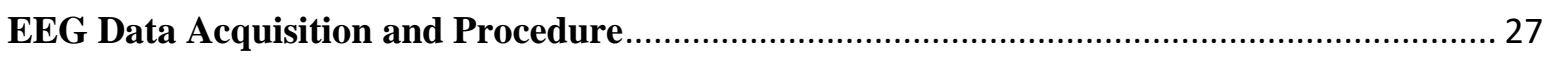

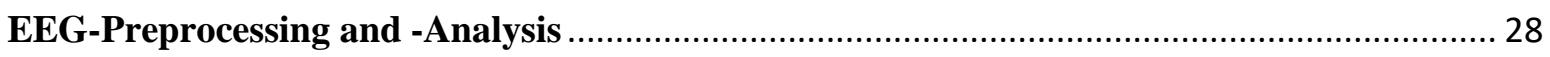

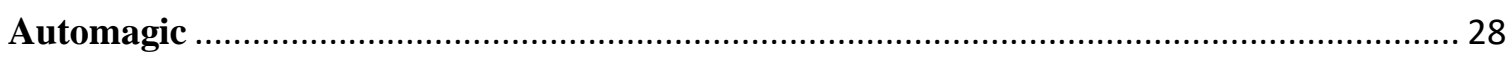

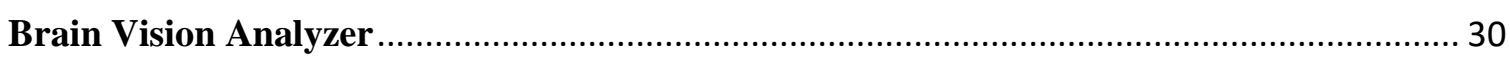

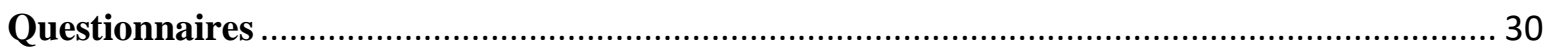

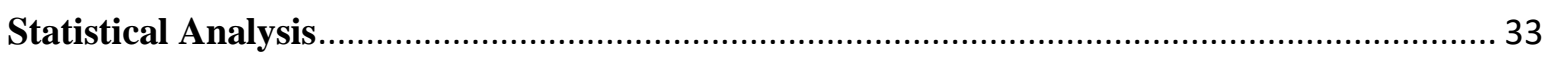

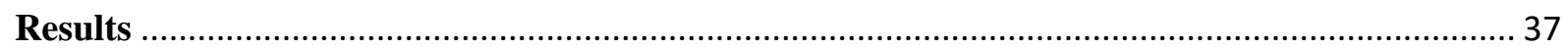

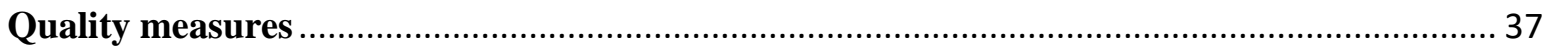

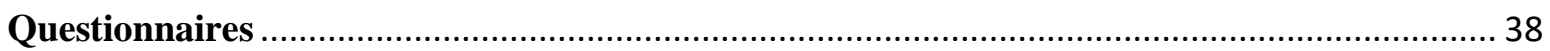

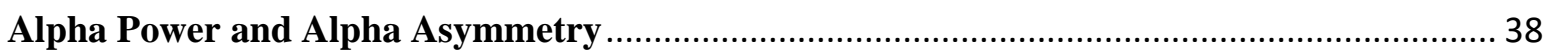

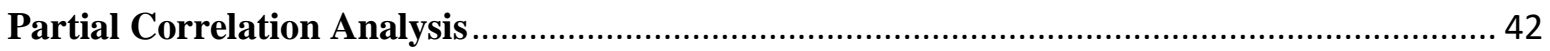

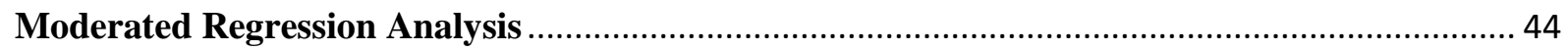

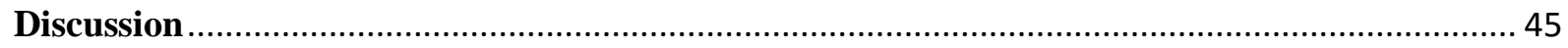

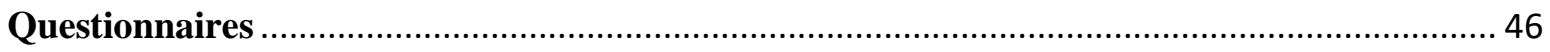

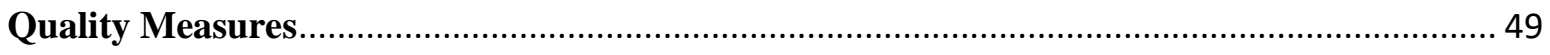

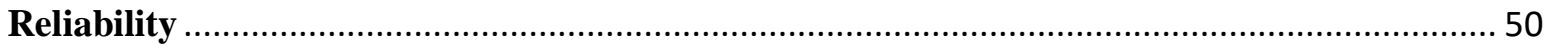

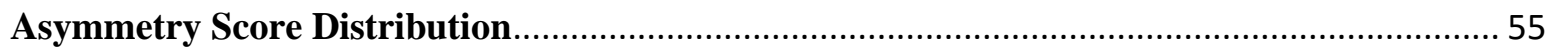

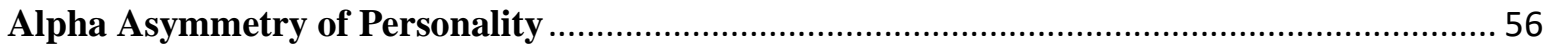

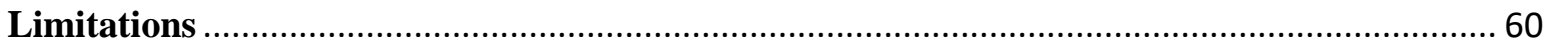

Implications for the \#EEGmanylabs Resting State EEG Asymmetry Project ........................... 63

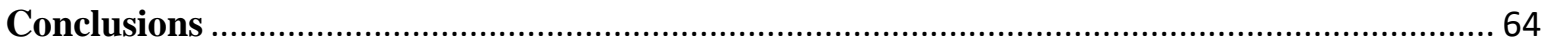

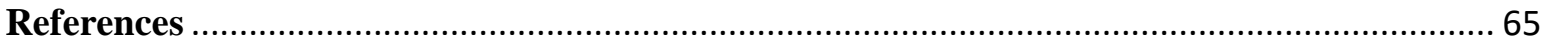

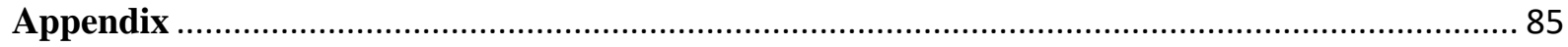

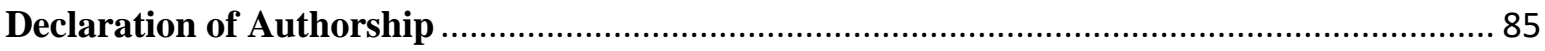




\section{Tables and Figures}

Tables

Table 1. Bivariate Spearman Correlation Coefficients between Self-Report Measures of Personality.... 40

Table 2. Split-Half Reliabilities (Spearman-Brown) for Average Alpha Power $(8-13 \mathrm{~Hz})$ and Asymmetry Scores

Table 3. Intraclass Correlation Coefficients for Alpha Power at Single Electrodes and Asymmetry Scores

Table 4. Partial Correlations (Spearman rank-correlation coefficients) between Self-Report Measures of Personality and Asymmetry Scores controlled for Handedness, Gender and Sleepiness.

Table 5. Moderated Regression Models for manually preprocessed EEG-data (EC). F3/F4Asymmetry Scores as Predictors of rBIS Scores. . .46

Supplementary Table 1. Partial Correlations between Personality Scales and Asymmetry Scores after Automated Preprocessing (EC-datasets).

Supplementary Table 2. Partial Correlations between Personality Scales and Asymmetry Scores after Automated Preprocessing (EO-datasets).

Supplementary Table 3. Partial Correlations between Personality Scales and Asymmetry Scores after Manual Preprocessing (EC-datasets).

Supplementary Table 4. Partial Correlations between Personality Scales and Asymmetry Scores after Manual Preprocessing (EO-datasets).

Figures

Figure 1. Asymmetry score generation in alpha asymmetry research

Figure 2. Flowchart for automatic (Automagic) and for manual (BVA) preprocessing ................31

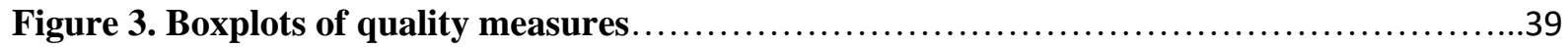

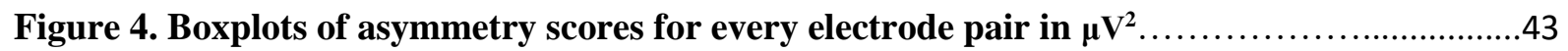

Figure 5. Interaction plot for the linear relationship between rBIS Scores (criterium) and asymmetry scores extracted from the electrode pair $\mathbf{F 3 / F 4}$ (predictor)

Figure 6. Johnson-Neyman plot for the interaction displayed in Fig.4 _.......................48

Supplementary Figure 1. Correlation Heatmap ....................................... 91

Supplementary Figure 2. Correlation Heatmap.........................................92

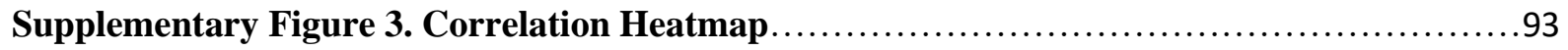

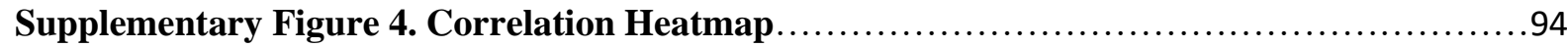




\begin{abstract}
Evidence suggests that replication of empirical findings in many fields of research is seldom warranted. As a result, \#EEGManyLabs Resting State EEG Asymmetry emerged to assess the replicability of landmark studies in psychophysiology. One potential factor that hinders replication is human subjectivity during EEG-preprocessing. To overcome this issue, the fully standardized preprocessing toolbox 'Automagic' (Pedroni, Bahreini, \& Langer, 2019) was developed. The goal of the present work was to execute a test-run on Automagic to help assess its adequacy for the project's purposes. Specifically, we examined the effect of automated preprocessing compared to traditional approaches on the analysis of frontal alpha asymmetries of personality. Forty-eight participants filled out the German BIS/BAS (Strobel, Beauducel, Debener, \& Brocke, 2001) questionnaire, as well as the German RST-PQ Pugnaghi, Cooper, Ettinger, \& Corr, 2018, among others. Next, we recorded 10 mins. of resting-state EEG twice, varying eye status (i.e., closed or opened) from each participant. All datasets were subsequently preprocessed in both Automagic and Brain Vision Analyzer. Measures based on signal amplitude as well as reliability estimates were computed to assess the quality of preprocessed EEG-data across methods. Furthermore, average alpha power from frontal, parietal, and occipital sites was extracted and used to compute asymmetry scores. Partial correlations between asymmetry scores and personality measures were computed, controlling for handedness, gender, and sleepiness. Additionally, we examined the moderating role of covariates using multiple regression analyses. Standardized preprocessing yielded better data quality in all considered aspects. No association between asymmetry scores and personality measures could be found, except when excluding influential data points. Employing different preprocessing approaches did not change our observations substantially. We consider standardized preprocessing a suitable option for large-scale multicentered replication projects. Our results support past research casting doubt on the robustness of the frontal alpha asymmetry of personality.
\end{abstract}

Keywords: alpha asymmetry, resting-state, EEG-preprocessing, artifact rejection, replication crisis, RST-PQ, BIS/BAS 


\section{Introduction}

Science is king (du Sautoy, 2017, p.1), and its crown jewels are the rigor and objectivity with which it accumulates unbiased knowledge. At least that is what any scientific endeavor strives for. But to achieve it, findings must be reproducible regardless of minor methodological tweaks to an original experiment or of the researcher carrying it out. This has been widely accepted in academic research for decades. In practice, it has been shown that several fields of research including psychology, biomedical sciences, cognitive science, and many more, are not meeting this requirement. A remarkable amount of empirical findings on which many models, theories, and even scientific careers rely on, are being called into question. The leading argument states that experiments are difficult to replicate. There are several reasons why this could be the case. Most prominently, experimental settings are hardly replicable because there is a lack of transparency in the methods used. Occluded methods also pave the way for many questionable research practices that effectively invalidate scientific inferences. This is detrimental to the public trust on research findings and on science at large.

Much criticism and reform proposals have already been made to tackle on the now called 'replication crisis'. Importantly, research dependent choices during design-, analyzing, and reporting stages of any study have been pointed out as a significant source of why empirical findings fail to reproduce. Methods to erode human subjectivity are much needed. A debate whether the psychophysiological literature may also be affected by these difficulties has been emerging in recent years (Larson \& Moser, 2017). Although thorough analyzes to support this claim are just emerging, there is a general acceptance within the academic community that this is the case. In this paper we will focus on research using electroencephalography (EEG) as the sole electrophysiological technique employed.

In psychophysiology, data preparation is subject to multiple arbitrary choices such as artifact rejection. Consequently, the final set of data analyzed can vary greatly depending solely on who is working on it. A standardized method for preparing data prior to analysis would be a step further towards more reproducible experiments and replicable results across studies and laboratories. The aim of the present work is to compare the capabilities of a fully automated pipeline to process EEG-data to those of more traditional approaches relying on human judgement. For this purpose, a set of resting-state EEG-data was related to asymmetry scores of psychological variables concerning affect, motivation, and personality to characterize the impact of both the automatic and the "manual" approach on the results. 
The following subsections summarize the most significant difficulties and developments in the past few years regarding research practices that ultimately strive for more reliable scientific knowledge.

\section{A Replication Crisis?}

The lack of popularity of replication studies in psychology up until the 2010s raised questions regarding the robustness of empirical findings (Lykken, 1991; Wiggins, \& Christopherson, 2019). First efforts to evaluate this issue developed. Klein et al. 2014. assessed the variation in replicability of classic and contemporary psychological effects from a total of 6344 participants. This required the recruitment of 36 different research locations willing to participate. Ten out of 13 studied effects showed signs of satisfactory replication. Moreover, this outcome was more dependent on the effect put to the test rather than on variability of sample and site characteristics (Klein et al., 2014). This further encourages world-wide, large-scale collaborations. A “many labs" approach to replication, as exercised by Klein and colleagues (For an example in developmental psychology see Frank et al., 2017).

A remarkable application of this concept came in 2015 when the Open Science Collaboration published one of the most ambitious projects dedicated to test the replicability of empirical findings in the psychological sciences (Open Science Collaboration, 2015). High powered direct replications of 100 influential studies on long-standing psychological phenomena were attempted. The results were staggering. Approximately only $36 \%$ of the replication attempts yielded a statistically significant result consistent with the original studies. Effect sizes halved. This seemed to sum up what debates had been speculating up until then: Psychology is in a 'replication crisis'. Consequently, doubt has been cast upon the credibility of empirical findings in the available literature. The authors ignited a variety of reactions from many field experts. In concert with the Open Science Collaboration, the Pipeline Project (Schweinsberg et al., 2016) reported failures to replicate ten moral judgement effects. Specifically, $40 \%$ of the original findings failed at least one major replication criterion (Schweinsberg et al., 2016).

On the other end of the debate, Patil, Peng, and Leek (2016) draw attention to the fact that many researchers as well as the general public misunderstand what failed replication attempts imply. They pose that failed replications do not necessarily invalidate the original finding. The resulting test statistics (e.g., a $t$-value, $F$-value, etc.) of a replication is subject to 
natural sampling variation that falls into a distribution. Small sample sizes, for instance, increase the degree with which a replication statistic can vary. Hence, being unable to reproduce a result should be regarded as a diagnostic of poor design of the original work, of the replication, or both, rather as evidence that the effect in question does not exist (Patil et al., 2016). In line with this conceptual framework, they found that the lack of replicability reported by the Open Science Collaboration is what should be expected (Patil et al. 2016). Alternative analyses, correcting for statistical errors in the 2015 paper, suggest that the replication rate is actually high (Gilbert, King, Pettigrew, \& Wilson, 2016).

Regardless of perspective, it has become apparent that the robustness of the psychological literature is not infallible. In the following section, reasons behind the apparent replication crisis are outlined as well as developments in recent years to counteract this situation.

\section{Current Scientific Practices: Flaws and Solutions}

Several sources for a lack of replicability have been identified and extendedly discussed. Most prominently, so called questionable research practices (QRP), researcher 'degrees of freedom' and the peer-reviewing system are in the locus of attention (Shrout, \& Rodgers, 2018). Simmons, Nelson, and Simonsohn (2011) exemplified many common statistical decisions (QRPs) implemented to manipulate empirical data, maliciously or not, to reach statistical significance, making a case for the psychological literature potentially being contaminated with false positive reports. Some of them include stopping data collection as soon as $p<0.05$, reporting dependent variables and covariates that foster statistical significance, and dropping groups or conditions to focus on big effects on a subset of the data (Shrout \& Rodgers, 2018; Simmons et al., 2011). Indeed, a survey by John, Loewenstein, and Prelec (2012) found that the majority of academic psychologists that participated admitted having used QRPs to some degree.

Part of understanding the popularity of QRPs means understanding the current publication culture in academia. Publications define to a great extent the course of scientific careers. Scientists experience great pressure to publish high-quality work and are expected to do so at a prolific pace. Publication rates as well as publication outlets serve as informal measures of prestige and value of an author's work. They influence hiring, promotions, and grant decisions (Miller \& Serzan, 1984). This leads to a conflict of interests: the motivation to pursue accurate high-quality research and the motivation to maximize publishability (Nosek, 
Spies, \& Motyl, 2012). Scientific journals dictate what content gets published thanks to the peer-review system. Crucially, a proneness from within the system to favor novel, statistically significant ideas as compared to already published, reexamined non-significant ones, known as the publication bias, has been identified (Francis, 2012a; 2012b). Replication studies, for instance, are deemed as uninteresting, uncreative, and even not essential, since they repeat findings already described in the past. This issue is best exemplified with Bem's (2011) infamous article Feeling the future published in the Journal of Personality and Social Psychology(JPSP). Alleged Evidence on 'precognition', the parapsychological phenomenon of retroactive influence on affect and cognition, was reported. In the following years, criticism in the form of papers providing evidence for a null effect after direct or conceptual replication attempts were submitted for publication without success. Many high-profile scientific journals including JPSP, condemned defying findings unworthy of publication for being mere replication studies (Wiggins, \& Christopherson, 2019). Thus, researchers find themselves in a system that incentives the use of QRPs. Combined with publication bias, these tendencies skew the literature toward inflated false positive studies.

In reaction to all shortcomings discussed above a new zeitgeist in academic psychology and related fields has been emerging. Beginning with Brian Nosek’s pioneering work in 2013 creating the Open Science Framework (OSF) and leading the establishment of the Center for Open Science, and the Transparency and Openness committee (Shrout, \& Rodgers, 2018), an era of more transparent publication culture is in sight. The OSF is a nonprofit organization that facilitates the sharing of study plans, materials, and documents (Shrout, \& Rodgers, 2018). Preregistration, the act of submitting design, methods, hypotheses, power calculations, and planned sample size to a reviewing process before data collection, is the cornerstone of the initiative. Preregistration effectively prevents numerous QRPs since every preregistered study is required to strictly follow the research proposal. Setting sample size declarations as a requirement promotes power analyses, which in turn hamper Type II errors. Moreover, raw data and code is made available to other researchers. This way, analyses by independent and impartial groups are a possibility. The Centre for Open Science encourages journals to add badges to authors practicing data sharing and preregistration in acknowledgement of their transparent work. Furthermore, the group developed the Transparency and Openness Promotion (TOP) Guidelines, originally formulated by Nosek and colleagues (2015). TOP guidelines specify the way in which journals ought to reinforce transparency in, data, analytic methods and code, research materials, design and analysis, study preregistration, analysis plan preregistration, as well as citation standards and 
replication. As of 2021, over 1000 journals have adopted these guidelines (Center for Open Science, n.d.)

As discussed earlier, other efforts aim for more collaborative research techniques. For instance, the Psychological Science Accelerator (PSA) is a multisite network of psychological researchers dedicated to enable and support crowdfunded research projects (Moshontz et al., 2018). Their mission is to accelerate the accumulation of replicable and generalizable evidence in psychological science by means of systematic data sharing (Moshontz et al., 2018). Their methods mitigate common limitations faced by individual research groups such as sample size and sample demographics and as a by-product present an opportunity for underrepresented subjects and researchers to take part in high-impact projects. Also, the organization's decentralized structure reduces the chances of individuals engaging in QRPs. The PSA follows the example of earlier work implementing the 'many labs' approach (Frank, 2014; Klein et al., 2014; O’Donnell et al., 2018; Open Science Collaboration, 2015, Schweinsberg et al., 2016).

\section{Beyond Psychology}

Monya Baker's 2016 survey published in Nature displays the overall notion of a replication crisis in science as perceived by 1576 researchers that filled out online questionnaires on reproducibility. However, although $52 \%$ of those surveyed believe in a crisis, $31 \%$ disagree that failed replication attempts are an indication of non-existent effects, even when failure at replication is common if not the norm (Baker, 2016). This trust on the available literature is nevertheless perilous. Yet another dramatic example comes from cancer biology. When trying to replicate 53 landmark studies, a mere $11 \%$ of the results coincided with the original studies (Begley, \& Ellis, 2012). As the authors noted, these findings underscore the fact that if science is to be carried out for the benefit of everyone, the highest standards of quality and rigor must be demanded (Begley, \& Ellis, 2012).

Fortunately, lessons learned from psychology reverberate in several related fields. Many of the issues discussed above also apply to cognitive neuroscience. As a result, interest in re-examining key findings is growing (DeLong, Urbach, \& Kutas, 2017; Ito, Martin, \& Nieuwland, 2017; Nieuwland et al., 2018). Low statistical power aggravates the already difficult task of making correct inferences from complex, noisy, multidimensional data. The most common reason for low statistical power is a small sample size. In MRI experimentation, for instance, sample sizes tend to be small because it is bound to high 
financial costs (Conrad, \& Bailey, 2020). Low power not only reduces the likelihood of not detecting a true effect but also increases the likelihood of finding false positives (Ioannidis, 2005). In 2015, the median fMRI study was only sufficiently powered to detect effect sizes of at least 0.75 , simply because there are usually not enough observations (i.e., subjects) per group or condition (Poldrack et al., 2017). Button et al. (2013) estimated the average power for the cognitive neuroscience literature to be between $8 \%$ and $31 \%$. It has been suggested that statistical power may be even lower than in psychology (Szucs, \& Ioannidis, 2017). Although this issue is well known, insufficient statistical power is still tolerated.

Furthermore, researcher degrees of freedom (Simmons et al., 2011) proliferate in cognitive neuroscience due to analyses requiring multiple steps. The workflow specifically chosen for a given dataset, the "pipeline", is a source of great result heterogeneity. Carp (2012) compared the results from a single MRI dataset analyzed with 6912 possible pipelines from the most popular software packages in current use and concluded that the workflow of choice determines to a great extent the brain activations found. Hence, minimal changes in analytical decisions potentially lead to different results. Botvinik-Nezer et al. (2019) offer an empirical example of this as pipeline flexibility and analytic liberty led 70 separate research teams using the same fMRI dataset to come to substantially different conclusions. Moreover, scientific reporting endorses concise language but at the price of obscuring many processing and analytical decisions (Poldrack et al., 2017). This not only increases the difficulty for researchers to attempt a replication but allows the use of QRPs. For instance, many exploration-driven findings may be reported as hypothesis-driven ones. This is also known as "Hypothesizing After Results are Known" or "HARKing" (Kerr, 1998). Poldrack et al. (2017) termed the neuroimaging version of it as "Selecting Hypothesized Areas after Results are Known" or "SHARKing".

Human psychophysiology is subject to the many methodological issues mentioned above as well (Baldwin, 2017). For instance, almost any experiment examining event-related potentials (ERPs) will yield significant outcomes because of vast noise in the data that randomly reached statistical significance if analysis parameters are chosen after having inspected the data (Luck \& Gaspelin, 2017). Specifically, researchers may engage in practices that Luck (2014) called "multiple implicit comparisons": the visual inspection of grand average waveforms resulting from an ERP experiment to look for time and scalp regions that may indicate any differences, say within- or between subjects, before carrying out main analyses. This procedure implies implicitly looking at thousands of data points across time and conditions only to later look at the same points explicitly via significant testing. Many 
reasons why specific sites and time frames were chosen can be found and presented such that it would seem like reasonable a priori hypotheses, i.e., HARKing (Luck, 2014; Luck \& Gaspelin, 2017). Although the publication guidelines of the Society for Psychophysiological Research state that "measurement windows and electrode site must be well justified" (Keil et al., 2014) not every published paper reports reasons for choosing their measurement parameters (Luck \& Gaspelin, 2017). It should be noted, that choosing spatial and temporal frames on theoretical grounds is oftentimes not feasible or just impossible (Fields, \& Kuperberg, 2020).

Statistical power is also believed to be alarmingly low. Although a priori sample size analyses help ensure sufficient power, this is not customary. Larson and Carbine (2017) randomly selected 100 clinically relevant EEG/ERP studies and assessed the frequency with which a priori sample size calculations were reported. None of them did. Thus, statistical robustness is not warranted probably due to neglect. To assess actual study power, Clayson et al. (2019) reviewed 150 randomly selected ERP studies published between 2011 and 2017 and estimated the mean sample size to be around 21 participants. Statistical power was around $.15, .50$, and .80 for small, medium, and large effect sizes, respectively (Clayson et al., 2019). Consistent with this notion, Baldwin (2017) concluded that it is reasonable to deem psychophysiology research as underpowered to even detect large effect sizes after calculating power as a function of many effect sizes and sample sizes of 51 studies in the last 2015 issues of Electrophysiology and the International Journal of Electrophysiology.

Furthermore, psychophysiology research includes many decision steps specific to the field that potentially create problematic flexibility (Baldwin, 2017). Here we consider two major distinctions integral to any EEG study to discuss them: Preprocessing and analysis. After data acquisition, raw EEG-data undergoes preprocessing. This consists of a series of procedures that transform the data into formats appropriate for main analyses. Some steps might include but are not limited to re-referencing, interpolation of missing data, segmentation, baseline removal, artifact rejection, artifact correction, channel rejection, and offline filtering (Keil et al., 2014; Li et al., 2020). Some techniques are heavily discussed in the literature for its reliance on human subjectivity. Most importantly, 'manual' noise reduction is a popular and necessary custom among researchers. This refers to inspecting the raw data visually and excluding single recording segments containing noise in the signal from further analyses. The most influential source of noise are artifacts. Artifacts may come from physiological activities such as eye blinks, saccades, muscular activity, skin potentials, and heartbeats, or from environmental interferences like electrode displacement, cable movement, 
power line, or recording equipment (Islam, Rastegarnia, \& Yang, 2016). Hence, manual EEG data cleaning mainly refers to rejection of gross artifacts. This procedure is nonetheless problematic for a number of reasons. Usually, entire timeframes containing artifacts are rejected even when artifacts might be observable on only one or few channels. Further, artifacts themselves can be a linear combination of signal and noise (Islam, et al., 2016). Thus, when excluding artifactual segments entirely, neural activity gets inevitably lost. To reduce data loss, blind source separation-based artifact correction is implemented. Blind source separation extracts unknown source signals from the raw recording. This technique assumes that the number of sources can be at most equal to that of observed channels. Independent component analysis (ICA), one of many source separation techniques, additionally assumes that different sources that compose the observed raw signal are independent. Algorithms separate recordings into single independent components (ICs) but most of them make no distinction between noise and signal. Human raters then inspect each individual component visually and decide whether to discard it or not before reconstructing the EEG data without excluded ICs. However, even highly artifactual components contain signal of interest to some degree. Thus, during data reconstruction distortion to the data is introduced. ICA is nonetheless exceptionally effective at identifying global artifacts (Urigüen \& Garcia-Zapirain, 2015) such as ocular artifacts (Flexer, Bauer, Pripfl, \& Dorffner, 2005) and possibly other physiological artifacts (Islam et al., 2016). ICA is the most widely implemented artifact correction method (Jang, Bian, \& Tian, 2019).

Manual rejection can be nonetheless time consuming and laborious. This process may take several hours across days to be completed. Consequently, the consistency with which artifacts are identified has been observed to vary between raters and over time for a single rater (Dawes, Faust, \& Meehl, 1989; Smith, Reznik, Stewart, \& Allen, 2017). This may compromise data reliability. Consensus between multiple raters is best when raters are experienced classifiers of particular artifacts (Viola et al., 2009) and when they have extensive training (Hatz, Hardmeier, Bousleiman, Rueegg, Schindler, \& Fuhr, 2015). Conversely, interrater reliability is low when signal components are classified dichotomously (e.g., brain activity vs artifact; Smith et al., 2017). The work by Shirk et al. (2017) investigated how discrepancies during preprocessing steps between three novice research assistants influence three well-established ERP effects during a face-name recognition memory paradigm (Ally \& Budson, 2007; Curran, 2000; Herron, Quayle, \& Rugg, 2003). All three effects were mostly robust against the assistants' subjectivity. The authors note that effect robustness may vary between electrodes. In their experiment, effects examined at frontopolar electrodes, as 
compared to parietal sites, showed the lowest and most variable interrater reliability coefficients ranging from 0.60 to 0.90 . (Shirk et al., 2017). Thus, regardless of conscientiousness and expertise human performance will inevitably vary. Depending on the research question and the regions of interest, even subtle differences in performance between various researchers could have an impact on the observed results.

Automated and semi-automated signal cleaning have been proposed to minimize/eradicate human subjectivity and unreliableness (Islam et al., 2016). If software were to successfully assume responsibilities of some portion of data processing with little to no human intervention, time and financial resources (e.g., research assistant employment and training) would be spared from the burden that comes from EEG data handling. Naturally, this raises the question whether algorithmic performance is commensurate with performance achieved under human supervision. Anecdotical evidence suggests that automatized artifact rejection, for instance, fails to identify artifactual components that human visual inspection would have whereas artifact-free segments, according to human judgement, are identified by automated tools as artifactual (Cohen, 2017). Comparing different analytical approaches, pipelines or toolboxes systematically, automatized or not, is no trivial task. To infer conclusions on the influence of single processing decisions it would be mandatory to hold all other factors (e.g., all other processing steps) constant. However, the catalogue of currently available methods is overwhelmingly diverse so that any two pipelines/toolboxes/software packages will almost inevitably differ from each other at various stages of data (pre)processing to some degree. Some efforts addressing these complications have found automated software to be indeed as reliable as the manual tradition and other standardized preprocessing techniques (da Cruz, Chicherov, Herzog, Patrícia Figueiredo, 2018; Hatz et al., 2015). Test-retest reliability has proved to be good on longitudinal data for both task-related and resting state EEG data (Cannon et al., 2012; Corsi-Cabrera, Galindo-Vilchis, del-RíoPortilla, Arce, \& Ramos-Loyo, 2007; McEvoy, Smith, \& Gevins, 2000). Other authors, however, have found discrepancy in EEG data after applying different preprocessing pipelines (Robbins, Touryan, Mullen, Kothe, \& Bigdely-Shamlo, 2020). Recent reliability assessments of resting state EEG data further indicated that reliability coefficients are dependent on the frequency band of interest (Suarez-Revelo, Ochoa-Gomez, \& Duque-Grajales, 2016). Interestingly, reliability was most robust for the alpha and theta bands (Suarez-Revelo et al., 2016). Thus, it is recommendable to test the influence that any newly developed toolbox exerts on raw EEG data ideally by comparing it to both human-dependent approaches and other established automated programs on a variety of datasets before implementing it on a 
formal study. Only this way we can assess how and to what extent variation in our results originates from data handling. There are several ways to implement this. For instance, software performance could be assessed at the analytical stage of a study.

Electrophysiological research usually examines some characteristic in the spectral and/or time domain. The impact of different preprocessing methods could be operationalized as the change of a specific measure, say an ERP component or a correlation between resting state data and a psychological variable. If different preprocessing methods yield similar results for a specific effect, it could be argued that they may be used interchangeably in subsequent replications. This approach was implemented here using the 'asymmetry score' (Smith et al., 2017), a popular index in EEG alpha asymmetry research (see below).

It should be noted that flexibility may also arise due to many liberties a researcher has at analytical stages. Choice of reference scheme, baseline correction periods, amplitude measures, difference scores, and electrode site scorings, to name a few, compose the basic analytical 'toolbox' of almost any EEG/ERP study. Every instance can be customized in a great variety of forms to fit analytical needs of complex research topics. However, great variety comes at a cost of high result variability. Sandre, Banica, Riesel, Flake, Klawohn, and Weinberg (2020) analyzed the impact of workflow choice on an analysis of the error-related negativity (ERN) component. The authors found that reliability measures, amplitudes measures, as well as the magnitude and direction of many effects were dependent on the combination of methods employed.

In summary, evidence suggests that analytical and preprocessing traditions in psychophysiology research compromise replicability. And yet, a both broad and rigorous examination of this issue is not available. However, multi-national efforts are in progress and will be discussed in the next subsection.

\section{\#EEGManyLabs}

The degree of reproducibility in psychophysiology remains an open question. To tackle on this issue the \#EEGManyLabs project was initiated (Pavlov et al., 2021). The main goal of the project is to assess the replicability of many landmark studies in psychophysiology which implement the scalp EEG as the primary neuroscientific method. Inspired by the 'many labs' approach coming from the psychological sciences (Frank, 2014; Klein et al., 2014; Moshontz et al., 2018; O’Donnell et al., 2018; Open Science Collaboration, 2015, Schweinsberg et al., 2016), the \#EEGManyLabs project will implement open science 
practices like pre-registration, registered reports, code and data sharing, and systematic documentation of analytical protocols (Pavlov et al., 2021). To attempt high-powered direct replications, large sample sizes will be gathered collectively by 'replication teams' consisting of an international network of collaboration sites. Effect size contrasts between replications and original papers, $p$-value distributions, Bayes factors, as well as standardized measurement error measures will quantify replicability (Pavlov et al., 2021). The potential modulating power of methodological differences on replication rates will be considered. The project's main repository is available online (https://osf.io/yb3pq/). Interestingly, prediction markets will be created to evaluate the EEG community beliefs on the likelihood of single studies replicating. This approach adds to Bakers' (2016) picture of the broad scientific community and its opinion on the ongoing replication crisis.

Three studies selected for replication (Coan \& Allen, 2003a; Harmon-Jones \& Allen, 1998; Wheeler, Davidson, \& Tomarken, 1993) concerning alpha band asymmetries were repurposed for the spin-off project \#EEGManyLabs Resting State EEG Asymmetry (Pavlov et al., 2021). The main goal in this subproject is to evaluate the replicability of the relationship between lateralized resting state EEG alpha band power and a variety of psychological variables such as affect, emotion and personality traits. To overcome the shortcomings from more manual preprocessing approaches discussed above, the recently developed fully automated preprocessing toolbox 'Automagic' (Pedroni, Bahreini, \& Langer, 2019) may be a viable option (see 'Methods' for a more detailed description). However, before incorporating the toolbox to the spin-off project it is recommendable to assess its adequacy beforehand. Thus, one main purpose of the present work is to run preliminary analyses on a smaller EEG resting state dataset to compare the toolbox's performance against manually operated preprocessing. In the spirit of replicating seminal psychophysiological findings, and since two of three asymmetry studies initially selected for direct replication are concerned with personality and motivation (Coan \& Allen, 2003a; Harmon-Jones \& Allen ,1998), the impact of preprocessing methods on the relationship between alpha asymmetry scores and personality measures are probed here. The next subsection summarizes major developments and setbacks in the theories of personality EEG asymmetry that reflect both their relevance to psychophysiology as a whole and the necessity to verify their replicability.

\section{EEG Alpha Asymmetries of Personality}

Hemispheric asymmetry is a fundamental property of functional brain organization in humans and other species (Ocklenburg, \& Güntürkün, 2017). Davidson, Schwartz, Saron, 
Bennet, and Goleman (1979) first reported detectable asymmetries using the EEG at frontal and parietal sites. The authors underlined the potential of their discovery in explaining interindividual differences in emotional processing and neuropsychological facets of various psychopathologies (Davidson et al., 1979). A new research field was born. The most basic and yet widely spread approach to EEG asymmetries consists of measuring the difference in brain activity between homological frontal sites on the left and right hemispheres as quantified by either total $\left(\mu \mathrm{V}^{2}\right)$ or density $\left(\mu \mathrm{V}^{2} / \mathrm{Hz}\right)$ power on the canonical $(8-13 \mathrm{~Hz})$ alpha frequency band (Allen, Coan, \& Nazarian, 2004; Coan \& Allen, 2003a; Hagemann, 2004). In a 10-20 system, this mostly refers to alpha power derived from electrode pairs F3/F4 and F7/F8, sometimes also F5/F6 (Harmon-Jones, Gable, Peterson, 2010). After estimating alpha power on single electrodes an asymmetry score is computed by subtracting the natural-log transformed alpha power of one left frontal electrode from its homologous right counterpart, e.g., ln F4 $\left(\mu \mathrm{V}^{2}\right)-\ln \mathrm{F} 3\left(\mu \mathrm{V}^{2}\right)$ (Figure 1; Allen, et al., 2004). Larger asymmetry scores reflect more alpha power on the right relative to the left and are interpreted as greater relative left than right cortical activity. Since alpha power dominates EEG recordings when participants do not engage in any particular task (i.e., during a 'resting state'), its presence has been equated to reduced cortical network activity (Reznik, \& Allen, 2018). This interpretation has been confirmed using source localization techniques (Pizzagalli, Sherwood, Henriques, \& Davidson, 2005). It has been suggested that alpha oscillations represent a desynchronization during local neural activation while other frequency ranges, like delta or theta, synchronize (Knyazev \& Slobodskaya, 2003). Thus, cortical activation results in lower alpha power (Neal $\&$ Gable, 2016). Asymmetry scores obtained during resting state EEG alpha power analysis has become a popular procedure since they are a unidimensional scale sensible for personality facets in behavioral responding and risk for psychopathology (Coan, Allen, \& McKnight, 2006). Analyses suggest that around half the variance in correlational evidence between resting state recordings and individual difference measures is due to trait influences and the other half possibly due to state influences (Hagemann, Hewig, Seifect, Naumann, \& Bartussek, 2005; Hagemann, Naumann, Thayer, \& Bartussek, 2002). Hence, state influences must also be addressed. This is usually done by experimentally evoking changes in psychological variables of interest and assessing their relation to asymmetric cortical activity (Allen, Keune, Schönenberg, Nusslock, 2018; For extensive reviews, see Smith et al., 2017; 
F3

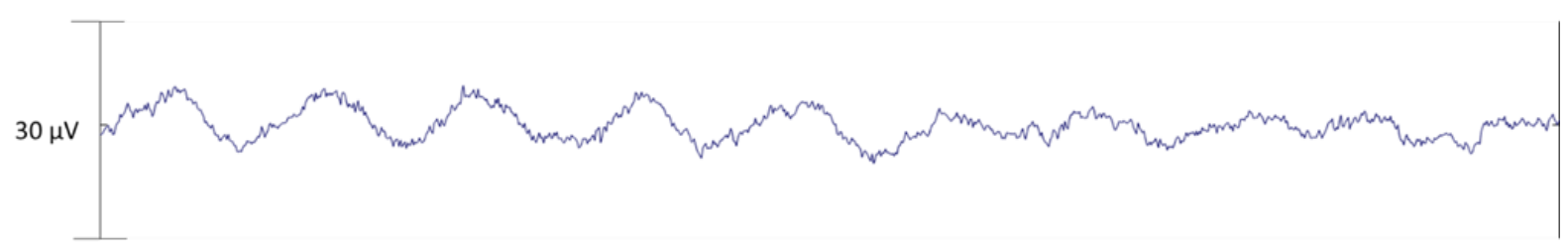

F4

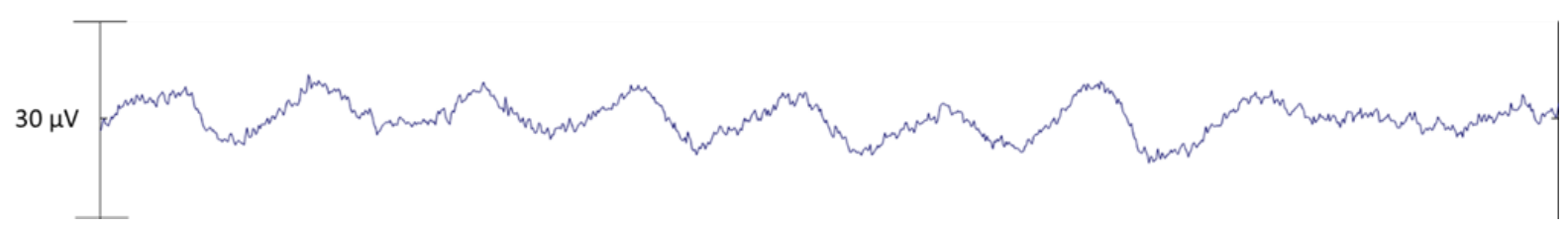

$\zeta^{\mathrm{FFT}}$
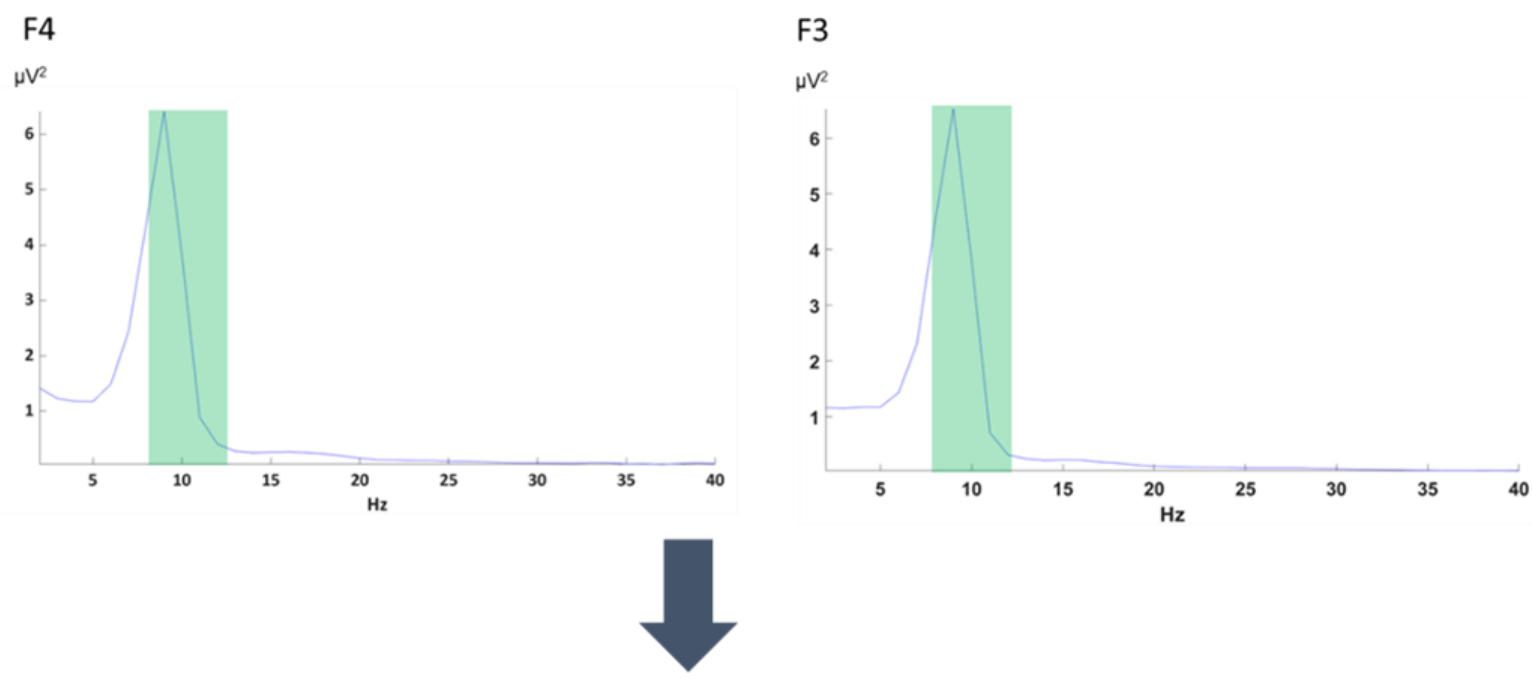

\section{$\operatorname{Ln} \mathrm{F} 4\left(\mu \mathrm{V}^{2}\right)-\operatorname{Ln} \mathrm{F} 3\left(\mu \mathrm{V}^{2}\right)$}

Fig.1.Asymmetry score generation in alpha asymmetry research. The upper panel depicts a 1-second epoch containing alpha rhythm of one participant at electrodes F3 and F4. The middle panel displays power $\left(\mu \mathrm{V}^{2}\right)$ plotted against frequency $(\mathrm{Hz})$ after an FFT applied on channels F3 and F4. Green segments mark the canonical alpha band $(8-13 \mathrm{~Hz})$ for which the average power is calculated. Average alpha power at a left-hem ispheric electrode (e.g., F3) is subtracted from average power at its right-hemispheric homologue (e.g., F4). The end product is the asymmetry score.

Harmon-Jones \& Gable, 2018; Reznik, \& Allen, 2018).

Initial evidence suggesting trait-like left-right alpha power differences (Tomarken, Davidson, Wheeler, \& Doss, 1992; Wheeler et al., 1993) or power differences elicited by affective stimuli (Ahren \& Schwartz, 1985; Davidson, Ekman, Saron, Senulis, \& Friesen, 1990; Reuter-Lorentz, \& Davidson, 1981) lead to the 'valence model' of emotional processing (Davidson, 1992). The model proposes that greater left than right brain activity, 
indirectly and inversely indexed by more right- than left alpha power, is related to the experience of positive emotions. Conversely, negative emotions are characterized by greater left than right alpha power (Tomarken et al., 1992). In spite of evidence supporting the valence model (Jacobs \& Snyder, 1996; Petruzzello \& Landers, 1994; Tomarken et al., 1992; Wheeler et al., 1993), Hagemann, Naumann, Becker, Maier, \&Bartussek (1998) called into question the model's validity due to methodological issues. They argue that the proposed relationships are dependent on analysis decisions such as reference choice, and the computation of asymmetry scores.

Davidson $(1993 ; 1998)$ further described asymmetry in behavioral and motivational direction. The approach/withdrawal model of alpha asymmetries posits that greater relative right frontal alpha power, as both a trait and a state, is observed when engaging in approaching behaviors elicited by appetitive stimuli and reward. Meanwhile, greater left frontal alpha power relates to a propensity to withdraw and disengage and elicited by aversive stimuli and punishment (Davidson, 1998; Coan \& Allen, 2003b). By far, the most popular measures used to investigate approach/withdrawal EEG asymmetries are the BIS/BAS scales developed by Carver and White (1994). The 20-item questionnaire is a psychometric measure of Gray's (1982) behavioral activation system (BAS) and behavioral inhibition system (BIS) of his reinforcement sensitivity theory (RST). Sutton and Davidson (1997) found greater right alpha power on subjects with greater scores on the BAS scale as well as greater left alpha power in subjects with higher BIS scores. Using standardized low resolution brain electromagnetic tomography, BIS scores have been related to greater relative rightward activity that originates in the dorsolateral prefrontal cortex (Shackman, McMenamin, Maxwell, Greischar, \& Davidson, 2009) and BAS scores to greater relative leftward activity coming from the middle frontal gyrus (DePascalis, Cozzuto, Caprara, Alessandri, 2013). It becomes evident that Davidson's valence model is closely related to his approach/withdrawal model since emotional valence is allowed to arise parallel to motivational tendencies. In his view, approach behaviors can be accompanied by positive emotions just as withdrawal behaviors by negative ones (Davidson, 1998). This makes the interpretation whether EEG alpha asymmetries are determined by motivational direction or emotional valence difficult. Compelling evidence that clarifies the roles of emotion and motivation in EEG asymmetries comes from research on anger, a negatively valenced emotion but with approach-eliciting motivational tendencies (Harmon-Jones, \& Gable, 2018). Indeed, BAS scores are positively correlated with trait anger (Harmon-Jones. 2003; Smits, \& Kuppens, 2005) and with more intense anger in response to situational anger manipulations (Carver, 2004). In an early study 
on anger and EEG asymmetry, Harmon-Jones and Allen (1998) found an association between asymmetry scores stemming from resting state recordings and trait anger in adolescents, as measured by the Buss and Perry (1992) Anger Questionnaire. Specifically, greater left frontal activity correlated positively with trait anger. This relationship has been observed in student populations (Harmon-Jones, 2004), in imprisoned violent offenders (Keune, et al., 2012), and adults with ADHD (Keune et al., 2011). Studies inducing anger experimentally also found support for the relationship between leftward neural activation and anger (Harmon-Jones, \& Sigelman, 2001), even using neurostimulation techniques such as repetitive transcranial magnet stimulation (d'Alfonso, van Honk, Hermans, Postma, \& de Haan, 2000), and transcranial direct current stimulation (Hortensius Shutter, \& Harmon-Jones, 2012). Experiments manipulating approach tendencies while holding positive affect constant showed that alpha asymmetries were highest when elicited approach motivation was also high (E. Harmon-Jones, C. Harmon-Jones, Fearn, Sigelman, \& Johnson, 2008). Hence, previous EEG asymmetry studies may have been confounding emotional valence with motivational tendencies (Harmon-Jones, Gable, \& Peterson, 2010).

The relationship between leftward frontal activity and the BAS/approach motivation is accepted to be robust and applies in a wide variety of situations. (Harmon-Jones \& Gable, 2018). Research investigating impulsivity, a psychological construct related to or overlapping with approach behavior has also found a relation to greater relative leftward activity in healthy individuals (Neal \& Gable, 2016). Studies in clinical populations have observed similar asymmetry patterns. Elevated left frontal activity in manic episodes compared to healthy controls has been reported (Kano, Nakamura, Matsuoka, Iida, \& Nakajima, 1992). Patients with manic/hypomanic symptoms with increased left frontal alpha power tend to take difficult challenges but with attractive reward outcomes more often than controls do (Harmon-Jones et al., 2008). The decline in self-control, also called ego depletion (Muraven \& Baumeister, 2000), putatively causes an increase in approach motivated behavior (Schmeichel, C. Harmon-Jones, \& E. Harmon-Jones, 2010). Nevertheless, the approach/withdrawal model of EEG alpha asymmetries also faces challenges imposed by contradicting evidence. Studies with large sample sizes have failed to observe the association between resting state EEG alpha power and BAS scores (Gable, Mechin, Hicks, \& Adams, 2015; Neal \& Gable, 2016; 2017; Wacker, Chavanon, \& Stemmler, 2010). More disputed is the association between the BIS/withdrawal and relative rightward neural activity. HarmonJones and Allen (1998) found a relationship between alpha power and approach motivation but not for withdrawal. Their results are in line with other failed attempts to reproduce the 
effect (De Pascalis, Cozzuto, Caprara, Alessandri, 2013; Coan \& Allen, 2003a). Conversely, evidence supporting associations between BIS sensitivity and alpha power is provided by only a handful of studies (e.g., Balconi \& Mazza, 2009, Balconi, 2011; Shackman et al., 2009; Sutton and Davidson, 1997, Quaedflieg, Meyer, Smulders, \& Smeets, 2015). To summarize, most past studies have either replicated the link between the BAS and left frontal activity but failed to find a relationship between the BIS and right frontal activity or failed to replicate both relationships with frontal asymmetric activity (Neal \& Gable 2017).

To explain inconsistencies in the literature, the original approach/withdrawal model has undergone many revisions and expansions. After Harmon-Jones (2003) clarified the interplay between emotion and motivational direction in asymmetrical frontal cortical activity, Coan and Allen (2003a) further highlighted theoretical flaws in Davidson's original model. Gray's original BIS construct is defined to be sensitive to conditioned aversive stimuli, omission/termination of expected reward, novelty, innate fear stimuli, suppresses ongoing operant behavior and increases arousal and attention in the face of threat (Corr \& McNaughton, 2008). Davidson's withdrawal construct does not map satisfactorily onto this conception of the BIS since the BIS primarily interrupts behavior and enhances vigilance but does not necessarily elicit withdrawal by doing so. Further, withdrawal behavior can be elicited by negative reinforcement to which the BAS is sensitive to (Coan \& Allen, 2003b). Coan et al. (2006) addressed these empirical and theoretical inconsistencies by proposing their 'capability model'. The capability model suggests that EEG alpha asymmetries are most sensitive to individual differences in motivational behaviors when subjects confront emotionally challenging situations at the time of recording. It is in these situations where subjects manifest their latent emotion-regulatory abilities, or capabilities (Coan et al., 2006). Johannes Hewig further published his bilateral BAS model, which argues that both the approach and withdrawal systems are subordinate to the higher-order BAS and depend on activation of frontal sites on both hemispheres (Hewig 2004, 2005, 2006). This model is in line with previous models of motivational direction and further explains weak relationships in studies using resting state EEG data as the basis for neural indexes. Rodrigues, Müller, Mühlberger, and Hewig (2018), allowed participants to move freely in a virtual task and found greater bilateral activation when participants were engaged in behavior compared to when they were doing nothing. Additionally, greater relative frontal activity on the left was associated with approach behavior whereas greater relative frontal activity on the right was associated with withdrawal behavior of any kind (Rodrigues et al., 2018). 
Yet another development in EEG alpha asymmetry theory came with Gray's major revision of RST (rRST; Gray \& McNaughton, 2000). In his rRST, three major systems that constitute the neurobiological foundation of personality are postulated: the revised BAS (rBAS), the revised behavioral inhibition system (rBIS), and the fight-flight-freeze system (FFFS) (Gray \& McNaughton, 2000). Gray’s BAS was originally postulated as a system sensitive to conditioned appetitive stimuli, activated by the presentation of stimuli associated with reward and termination or omission of signals of punishment (Corr \& McNaughton, 2008). The BAS underwent minor changes that are not believed to have an impact on the current alpha asymmetry literature. The FFFS took over functions under previous jurisdiction of the BIS and expanded its faculties by mediating reaction to all aversive stimuli, conditioned or unconditioned. The FFFS is proposed to mediate the emotion of fear. As such, the FFFS coordinates defensive behavior against threat such as avoidance, freezing or fight in the face of inevitable confrontation (Corr \& McNaughton, 2008). The original BIS (Gray, 1982) was redefined in critical ways that might take alpha asymmetry theory in barely explored directions. rBIS is postulated to mediate anxiety, rumination, and feelings of worry. Most critically, rBIS is responsible for the resolution of concurrent goal conflict that occurs when the other systems are activated simultaneously and elicit contradictory behavior (Corr \& McNaughton, 2008). For example, in foraging situations where animals explore the environment searching for loot (i.e., approach behavior) but simultaneously exposing themselves to attacks, the urge to retreat (i.e., withdrawal behavior) interferes. In this case, the rBIS putatively increases vigilance and readiness to escape in case of unexpected threat. This way the rBIS enables flexibility in behavior by dynamically regulating the behavioral output as a result of inhibiting one system or the other depending on the situation's demand (Corr \& McNaughton, 2008). It should be noted that goal conflict within the same systems (BAS/BAS- and FFFS/FFFS-conflict) are considered possible and are also resolved by the rBIS. Further rBIS functions comprise effortful control, constraint, self-control, inhibition, and error detection (Gray \& McNaughton, 2000)

Hence, rRST no longer links the BIS to withdrawal dispositions and behavior but rather to regulatory control of motivational direction. If rRST is correct, past research focusing on right frontal cortical activity might have been confounding withdrawal with regulatory control. After all, the majority of research in this field is based on relationships between EEG recordings and the BIS/BAS scales of Carver and White (1994). The BIS scale is sensitive to the unrevised BIS, which is a conflation of FFFS and rBIS in rRST. Although not substantial, there is a corpus of research supporting rightward cortical activity being 
allocated on the right hemisphere. This is reflected in research examining personality traits associated with inability to regulate motivational tendencies (Gable, Neal, \& Threadgill, 2018). Greater relative left frontal activity has been related to positive urgency, a measure of impulsivity reflecting failure to inhibit approach urges, i.e., deficient rBIS functioning (Gable et al., 2015). Importantly, results using standardized low-resolution brain electromagnetic tomography indicated that this asymmetric relation is due to reduced activity in the right inferior frontal gyrus. This relationship holds true even controlling for approach motivation (Neal \& Gable, 2016). Similarly, trait impulsivity but not approach motivation relates to reduced right frontal activity toward alcohol cues (Mechin, Gable, \& Hicks, 2016). Enhanced risk-taking behavior is predicted by diminished baseline activity of the right lateral prefrontal cortex (Gianotti et al., 2009). Hence, reduced rightward frontal activity rather than increased leftward activity could explain enhanced disinhibition (Gable et al., 2015). There is also evidence that relates rBIS to conflict detection in response to errors. Increases in neural correlates of error detection such as the ERN and the N2 ERP have been found in individuals ranking high in behavioral inhibition, anxiety, and emotion regulation ability (Amodio, Master, Yee, \& Taylor, 2008; Proudfit, Inzlicht, \& Mennin, 2013). Conversely, Nash, Inzlicht, and McGregor (2012) further found that greater relative left frontal activity and relative right frontal activity predicted reduced and increased ERN amplitude, respectively. This suggests that individuals with greater relative right frontal activity may be more concerned with making mistakes and with punishment. Taken together, there is initial evidence indicating a relation between rBIS and relative right frontal activity.

The work of Jan Wacker and colleagues (2003, 2008, 2010) integrates empirical findings linking inhibitory control to the right hemisphere in his BIS/BAS model of anterior asymmetry (BBMAA). In this framework, the BAS is allocated in the left hemisphere and the BIS, compatible with the rBIS, to the right. Interestingly, the FFFS functioning, comprising avoidance motivation, is reflected in greater relative left frontal activity. To test the model, avoidance motivation must be disentangled from approach-avoidance conflict (Rodrigues et al., 2018). That is, FFFS must be activated independently from rBIS experimentally. This is no trivial task since every system in RST is typically co-activated with another to some degree (Corr \& McNaughton, 2008; Gable et al., 2018). Few studies have sought to explicitly disentangle the FFFS/rBIS confound in humans. Lacey, Neal, and Gable (2020) let participants step away from aversive auditory stimuli (i.e., withdrawal behavior) for no reward or be exposed to it in exchange of reward (i.e., approach/withdrawal conflict). Greater relative right frontal activity was related to the conflict-eliciting condition whereas pure 
withdrawal was not (Lacey et al, 2020). More recently, in a study by Lacey and Gable (2021) participants indicate the likelihood of viewing negative (vs. positive) images for no points that served as reward (avoidance only condition), and negative (vs. positive) images for the chance to win points (approach-avoidance conflict conditions). Participants exhibited greater relative right frontal asymmetry as measured with EEG while making percent likelihood selections in the approach-avoidance conflict conditions relative to the avoidance only conditions. The greater the approach withdrawal conflict, the greater the rightward asymmetry (Lacey, \& Gable, 2021). Together, these results suggest that motivational conflict, and not withdrawal/avoidance motivation, is associated with greater relative right frontal activity.

To corroborate BBMAA predictions, traditional correlational analyses between personality traits operationalized via self-report measures with alpha power extracted from resting state EEG recordings are an additional option. It is primordial for this approach to select appropriate psychometric measures. Recently, the Reinforcement Sensitivity Theory of Personality Questionnaire (RST-PQ) was created to specifically have a research tool sensitive for the central systems postulated in rRST (Corr, \& Cooper, 2016). To our knowledge, there are only two studies that have examined the link between RST-PQ scales and resting state EEG asymmetries directly (De Pascalis, Sommer, \& Scacchia, 2018; De Pascalis, Cirillo, \& Vecchio, 2020). One study found a significant relationship between one rBAS subscale reflecting impulsivity and greater relative right hemispheric alpha power. Interestingly, the same association was found for the FFFS whereas the rBIS did not correlate with alpha (De Pascalis et al., 2018). In the second study, only the rBAS, but neither the rBIS nor the FFFS showed any correlation to asymmetry scores (De Pascalis et al., 2020).

In summary, no consensus over the many theoretical accounts has been reached. Moreover, none of them can dissolve empirical inconsistencies entirely. A recent metaanalysis considering methodological and theoretical heterogeneity discussed above could not find support for alpha asymmetries in personality traits (Kuper, Käckenmester, \& Wacker, 2019). A systematic review focusing on motivational personality traits (approach/withdrawal) and resting state EEG alpha asymmetry concluded that this field of research yields unstable results (Vecchio \& De Pascalis, 2020). Hence, the replicability of the alpha asymmetries of personalities is yet to be clarified, even though alpha asymmetry of personality is nowadays common textbook knowledge (Carver \& Scheier, 2012; Mischel, Shoda, \& Ayduk, 2007; Ocklenburg \& Güntürkün, 2017; Reeve, 2014) and still a highly active area of research (Reznik \& Allen, 2018). 


\section{Summary of Section and Hypotheses}

Replication in many research branches has not received enough attention. Inspired by the pioneering work in psychology, other fields of research are initiating efforts to assess the replicability of their own empirical findings. A lack of replication in human electrophysiology is suspected but not yet examined. EEG research has been proposed to suffer from similar methodological and analytical shortcomings common to many other fields in the biomedical and human sciences. Additionally, faulty practices specific to the field may contribute to result variability. \#EEGManyLabs is the first systematic multinational open science-oriented initiative trying to diagnose replication rates in human electrophysiology (Pavlov et al., 2021). Three highly influential papers of the study catalogue in the focus of this project concern alpha band asymmetries related to emotional and motivational personality traits (Coan \& Allen, 2003a; Harmon-Jones \& Allen, 1998; Wheeler et al., 1993). As a result, the spin-off subproject \#EEGManyLabs Resting State EEG Asymmetry was created to focus solely on this area of the EEG literature (Pavlov et al., 2021). Standardized procedures during preprocessing and analysis stages of any study are desirable to ensure result comparability between collaborating labs. Automated software is a promising option. Automagic (Pedroni et al., 2019) is a fully automated preprocessing toolbox currently taken into consideration for the spin-off project. The rationale behind the present work is to conduct pilot analyses of resting state EEG data using Automagic to assess its suitability for large scale 'many labs'-inspired (Klein et al., 2014) replication attempts. Specifically, multiple characteristics of automated preprocessed data are compared to and contrasted with data obtained with traditional manual preprocessing procedures (see 'Methods'). A further goal was to characterize the impact of preprocessing (manual vs automatic) on resting state EEG alpha asymmetries of traits in motivational personality. Lastly, inconsistencies in the alpha asymmetry literature were addressed.

We compared various characteristics of EEG-data based primarily on variance of signal amplitude to assess the effect on data quality yielded when employing different preprocessing methods. Since manual and automatic preprocessing approaches may differ in methodology considerably, we expected to observe differences in data quality.

Since characteristics of EEG-data may turn out differently when different researchers and/or different preprocessing programs handle the same datasets independently, we decided to investigate the degree of variability by calculating interrater reliability measures. These measures were computed for alpha power and asymmetry scores (see next section). In 
agreement with past research (Hatz et al., 2015), we hypothesized that reliability for both measures between raters and between programs is acceptable. Interrater reliability and crossmethod reliability should not differ from each other. We base our expectations on the work by Hatz and colleagues (2015) where interrater reliability (Kappa =0.75) and cross-method reliability $($ Kappa $=0.78)$ were at similar levels during the baseline measurements of their longitudinal design. We also assessed the reliability of both asymmetry scores and alpha power per se by calculating split-half reliability estimates. Both measures have been found to be highly reliable in the past (Hagemann, 2004, Allen et al., 2004). Thus, we expected to observe a similar pattern here.

We were interested in exploring the impact of preprocessing on the most crucial unit of measurement in alpha asymmetry research: the asymmetry score. To this end, we first examined how different preprocessing approaches might influence the polarity of asymmetry scores (e.g., rightward asymmetry, leftward asymmetry, or no asymmetry at all) at every electrode pair of interest. We did not have any expectations on the direction of the putative effect (e.g., sign change, magnitude change). We nevertheless hypothesized, once more, differences as a result of dissimilar preprocessing approaches. This also applies to an additional examination we carried out where we compared the distribution between asymmetry scores extracted at different electrode pairs.

We further propose here that replication failures in alpha asymmetry research, direct or conceptual, are partially due to the use of personality scales that do not properly tap on underlying neurobiological systems. First, psychometrical distinctiveness among all selfreport measures must be established. For this, we investigated whether each scale indeed relates to every other scale as theoretically predicted. rBAS subscales were expected to correlate strongly with one another as well as with an aggregated rBAS but not with FFFS or rBIS. Considering the theoretical conception of rBIS and FFFS, we predicted weak to moderate correlations between the two constructs. Classic BIS and BAS should show no relationship either. Considering the negligible theoretical differences between rBAS and BAS, a moderate to strong relationship should be observed. Conversely, BIS and rBIS were not expected to correlate since this system suffered drastic changes in RST and are therefore psychometrically different constructs as well. If these predictions are correct, we can assume that our scales show discriminant and convergent validity, which in turn allow us to properly discuss competing theories in frontal alpha asymmetry research. 
Appropriate measures should relate more strongly to asymmetrical EEG alpha power. First, we expected the BAS in both classic BIS/BAS (Carver \& White, 1994) and RST-PQ scales to show a positive relationship with asymmetry scores. In the light of recent investigations (De Pascalis et al., 2018), greater FFFS scores as measured by the RST-PQ were also expected to positively correlate with asymmetry scores. A negative relationship was expected for rBIS whereas for Carver and White's BIS (1994) no significant relation to alpha asymmetry at all was expected. After all, greater relative right cortical activity may index inhibitory control rather than avoidance/withdrawal. Consequently, most of past research has failed to observe any relationship between classic BIS and alpha asymmetry (Gable et al., 2018). Additionally, we investigated whether these associations are exclusively frontal by also extracting asymmetry scores from parietal and occipital electrode pairs and relating those to personality measures as well. Given that the vast majority of alpha asymmetry research reports frontal asymmetry (Reznik, \& Allen, 2018; Harmon-Jones, \& Gable, 2018), we expected no exception here.

Past research has shown that many sample characteristics as well as situational variables may mask these associations (De Pascalis et al., 2018, 2020; Wacker et al., 2010). Gender (De Pascalis et al., 2018, 2020) and handedness (Ocklenburg et al. 2019; Packheiser et al. 2020) have an impact on alpha band asymmetry, and sleepiness is directly related to alpha rhythmicity (Åkerstedt \& Gillberg, 1990; Kaida et al., 2006). We considered all three variables and expected them to relate to asymmetry scores and consequently alter their relationship to personality traits.

\section{Methods}

The following section provides a description of participant recruitment, experimental procedure, software settings for EEG preprocessing and analysis, questionnaires, as well as statistical handling of data.

\section{Participants}

Potential participants were screened online to assess eligibility. Participation was denied if at least one of the following exclusion criteria was fulfilled: past or current mental/neurological disorders, sleep disorders, medication intake, regular and/or acute psychoactive drug consumption as well as not having reached adulthood. In the light of the 
ongoing SARS-COV-2 pandemic, participation was also denied to anyone presenting symptoms indicative of Covid-19 briefly before their appointment or if their health were gravely jeopardized in the event of an infection. Forty-eight participants were recruited. Due to technical problems, a significant portion of EEG-data during recordings with open eyes (see below) of two participants turned out unusable. We excluded these datasets from analysis but not all data coming from these participants altogether. Moreover, EEG-data acquisition could not be completed for one participant due to unsatisfactory impedance reduction. All data provided by this participant was discarded. Hence, 47 participants (14 males, 38 righthanders) constituted the sample of the present study (18-45 yrs, $M=23.723, S D=5.033)$. Participants submitted written informed consent prior to participation. The study was approved by the ethical committee of the psychology faculty at the Ruhr-University Bochum in accordance with the Helsinki Declaration. After completing all study tasks, participants obtained either a monetary compensation or course credit.

\section{EEG Data Acquisition and Procedure}

Data were acquired conjointly with a parallel project focusing on EEG alpha asymmetries of depression and anxiety. Hence, participants were required to fill out a variety of self-report measures in topics ranging from handedness, sleepiness, affective valence, depression and anxiety symptoms to personality and motivation. The present work focuses on self-report measures of motivational personality. Therefore, only subject-related scales will be discussed here (see Questionnaires). Specifically, scalp EEG was recorded using a 64electrode actiCap system (Brain Products GmbH, Munich, Germany) with a $1000 \mathrm{~Hz}$ sampling rate. Electrodes were placed on the scalp according to the 10-20 system. The cap consists of 56 lateral electrodes (Fp1, F3, F7, FT9, FC5, FC1, C3, T7, TP9, CP5, CP1, P3, P7, O1, O2, P4, P8, TP10, CP6, CP2, C4, T8, FT10, FC6, FC2, F4, F8, Fp2, AF7, AF3, F1, F5, FT7, FC3, C1, C5, TP7, CP3, P1, P5, PO7, PO3, PO4, PO8, P6, P2, CP4, TP8, C6, C2, FC4, FT8, F6, AF8, AF4, F2), 8 midline electrodes (AFz, Fz, Cz, CPz, Pz, POz, Oz, Iz), a reference electrode anterior to $\mathrm{Cz}$ and a ground electrode anterior to $\mathrm{AFz}$. Impedances were kept below $2 \mathrm{k} \Omega$.

Participants were instructed to sit still and relaxed in a state of mind wandering in an electrically shielded EEG room. Two separate resting state EEG recordings per participant were obtained. 10 minutes with eyes closed (EC) followed by 10 minutes with eyes open (OP) were recorded. For EO-recordings, participants were asked to rest their sight on a turned off 
computer monitor standing approximately $20 \mathrm{~cm}$ away from their head. Light sources were eliminated for the EC-condition. During EO-recordings lights were kept on in the EEG room. They were generally informed that resting state EEG served to investigate EEG correlates of personality traits without any further information about the specific aims of the study or hypotheses.

\section{EEG-Preprocessing and -Analysis}

All raw data collected were preprocessed and analyzed using Brain Vision Analyzer 2.0. (BVA, Brain Products GmbH, Munich, Germany), and Automagic v.2.0. separately (Pedroni et al., 2019). The following two sections outline the settings selected for each software to make preprocessing pipelines fairly comparable to each other and leave human judgement during artifact rejection, bad channel identification/interpolation and exclusion of ICs as the only meaningful differing preprocessing steps between the two. The manual procedure is represented in all processes involving BVA. For the rest of the present work, we will handle 'BVA'- and 'manual'-related terms interchangeably.

\section{Automagic}

Automagic is a fully automated MATLAB-based preprocessing toolbox. It was developed to improve on well-known shortcomings in preprocessing methods that foster QRPs and make big, longitudinal, heterogenous samples datasets difficult to manage. Only settings employed in our dataset well be discussed in this subsection. The present work was preprocessed using Automagic in MATLAB 2021a (The MathWorks Inc., Natick, Massachusetts).

In a first step, channels with low signal-to-noise ratio or very low or no signal throughout a considerable time span of the EEG recording were identified. This is achieved by applying the EEGLAB plug-in clean_rawdata(). Specifically, channels with a ztransformed noise-to-signal value higher than 4 were marked. The same applies to channels with a correlation to their own robust estimate (random sample consensus reconstruction) lower than .85 for more than $40 \%$ of the time and to channels with no signal variation for periods longer than 5 seconds. The remainder of channels are filtered with a forwardbackward filter with a transition band of $0.25 \mathrm{~Hz}$ to $0.75 \mathrm{~Hz}$. Additionally, Automagic marks channels with a datapoint standard deviation higher than $25 \mu \mathrm{V}$. Single datapoints exceeding $100 \mu \mathrm{V}$ are omitted. However, if 50\% of channel recording contains datapoints exceeding 100 
$\mu \mathrm{V}$, these channels are marked as well. All marked channels are spatially interpolated using spherical splines (Perrin et al., 1989) on user's command at the end of Automagic's workflow.

Filtering was achieved by EEGLAB's pop_eegfiltnew() function. Specifically, a notchfilter for European line-noise frequency $(50 \mathrm{~Hz})$ and a high-pass filter of $0.5 \mathrm{~Hz}$ were applied.

Automatic artifact correction was achieved by applying the ICA run by ICLabel (PionTonachini, Kreutz-Delgado, Makeig, 2019), which is incorporated in Automagic. Specifically, the ICA uses the restricted infomax algorithm of Bell and Sejnowski (1995). Data was high pass filtered $(1 \mathrm{~Hz})$ to optimize the ICA's effectiveness (Pedroni et al., 2019). Note that this filtering is only temporary and specifically for the ICA. This was not applied to the final preprocessed data. Components with $80 \%$ probability of being labeled as cardiac, ocular, or muscular activity as well as channel noise and line noise were excluded.

Every dataset has a unique degree of artifact contamination. Consequently, the number of identified and interpolated bad channels, as well as the amount of recording excluded will inevitably vary between datasets. Automagic computes a set of objective quality measures for every dataset it preprocesses. This allows to quantify the degree to which any single dataset was altered after acquisition and before analysis.

Ratio of bad channels $(R B C)$. RBC describes the proportion of channels identified to need interpolation. The more channels that are interpolated the more of the signal is lost. More signal lost is interpreted as worse data quality.

Ratio of data with overall high amplitude (OHA). OHA is defined by calculating the ratio of data points across all channels that have a higher absolute voltage magnitude than a certain threshold.

Ratio of timepoints of high variance (THV). Similarly, the ratio of time points is identified in which the standard deviation of the voltage measures across all channels exceeds a certain threshold.

Ratio of channels of high variance $(\mathrm{CHV}) . \mathrm{CHV}$ is the ratio of channels for which the standard deviation of the voltage measures across all time points exceeds a certain threshold.

Following the recommendations of Pedroni et al. (2019), for EEG data high pass filtered with a bandpass filter of $0.5 \mathrm{~Hz}$ a standard deviation threshold of $15 \mu \mathrm{V}$ and an amplitude threshold of $30 \mu \mathrm{V}$ were implemented here for the entire sample. We argue that these settings are reasonable since wave peak-to-peak amplitudes during recordings in adult 
humans where alpha frequencies dominate are most typically observed within a range from 20 $\mu \mathrm{V}$ to $60 \mu \mathrm{V}$ (Orrison, 1995). Once a user commits to specific thresholds, Automagic considers all resulting quality measures and rates every file, usually every single EEGrecording, as either 'good', 'ok', or 'bad'.

For every dataset one value for every quality measure, i.e., one for every subject and condition, is obtained. With these scores differences in resulting data quality between human and automated performance were evaluated (see 'Statistical Analysis').

A 1-45 Hz band pass filter was applied before segmenting preprocessed EEG data into 1s-epochs. Spectral analysis was performed over a $2-40 \mathrm{~Hz}$ frequency band extracted by applying a Fast-Fourier Transformation with a Hanning window function.

\section{Brain Vision Analyzer}

All raw data was manually inspected by two independent experimenters (henceforth 'raters'). Channels with poor signal quality were interpolated topographically. Data segments considered to be predominantly artifactual were rejected via visual inspection. A $50 \mathrm{~Hz}$ zero phase shift Butterworth filter was applied at a slope of $24 \mathrm{~dB} /$ octave. A restricted infomax ICA was run to detect and eliminate ICs containing artifact sources stemming from saccades, eye blink, cardiac activity as well as any other component deemed implausible to be generated by neural activity.

Preprocessed data were re-referenced to the average of all electrodes. Next, a bandpass filter 1-45 Hz was applied. Continuous EEG recording were segmented into 1s-epochs. Spectral analysis was derived by means of the Fast Fourier Transformation. Epochs were tapered with a Hanning window function, with epochs overlapping to $10 \%$. Figure 2 summarizes all preprocessing steps undertaken in the present study for both preprocessing methods.

\section{Questionnaires}

Edinburgh Handedness Inventory. Handedness has been observed to predict alpha asymmetry (Ocklenburg et al. 2019; Packheiser et al. 2020). Hence, Handedness could act as a confound if it is not statistically controlled for. Participants' handedness was therefore assessed using the Edinburgh inventory (Oldfield, 1971). The lateralization quotient (LQ) was determined following the formula $\mathrm{LQ}=[(\mathrm{R}-\mathrm{L}) /(\mathrm{R}+\mathrm{L})] * 100$, with sum right-hand 

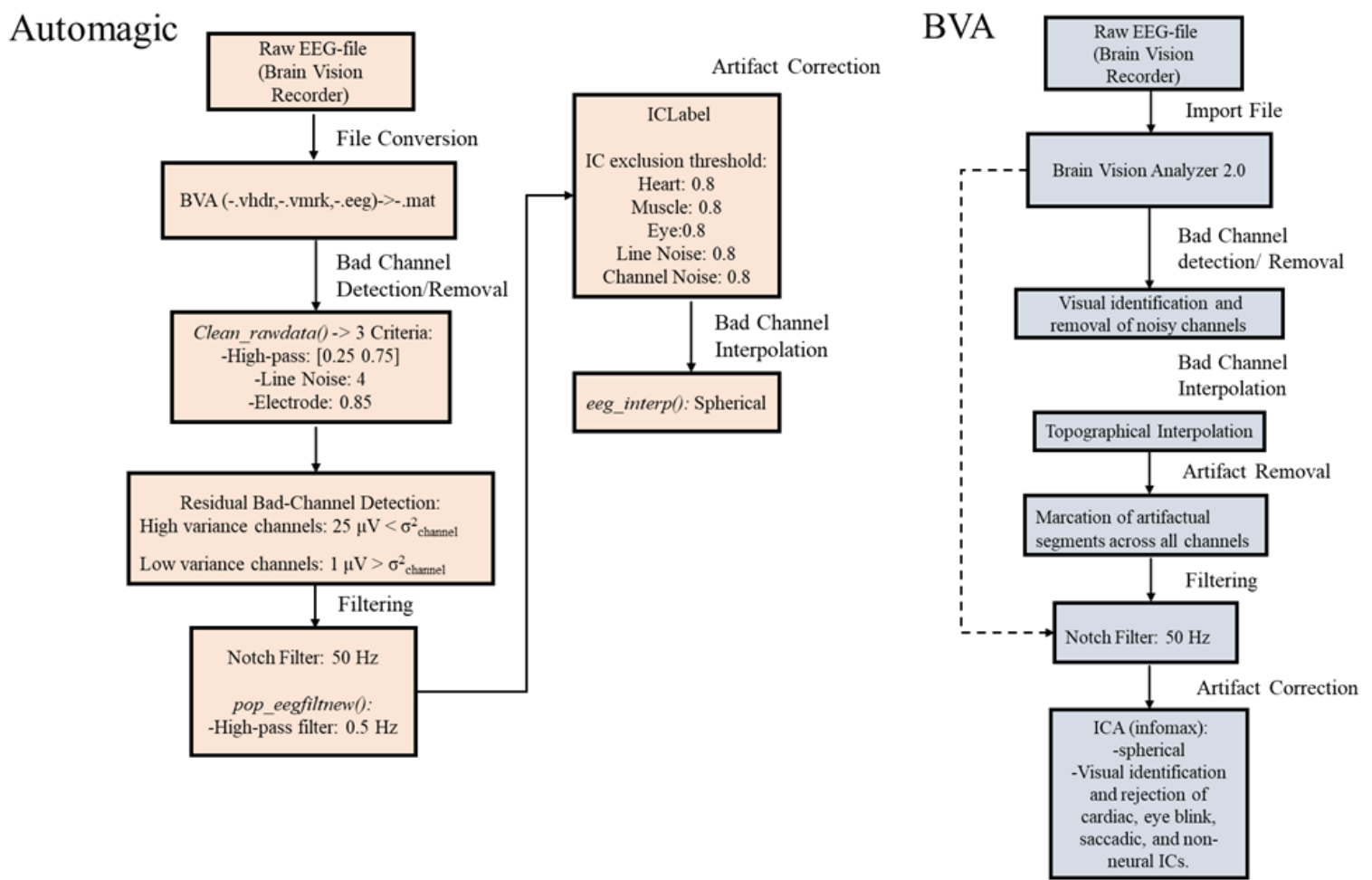

Fig.2. Flowchart for automatic (Automagic) and for manual (BVA) preprocessing. Preprocessing steps are sequential (filled lines). Since raw EEG -data was recorded using Brain Vision Recorder, data must be converted into a form at supported by Automagic first (e.g. format types that can be imported with EEGLAB) to import data successfully. Bad channel detection and rejection was achieved using EEGLAB's Clean_rawdata()-function integrated in Automagic based on three param eters followed by further detection and rejection of channels employing high - and low-variance thresholds. Line noise was elim inated applying a notch filter adequate for typical European power lines. Next, artifact rejection was achieved using ICLabel. ICs for different non-neural sources were rejected with a probability threshold of $80 \%$. Notice that no rejection of data-segments was carried out. Finally, removed bad channels were interpolated using a common spherical approach provided by EEGLAB's pop eegfiltnew(). Manual preprocessing using BVA started with a visual search for corrupt channels. If no poor-quality channels were identified during manual preprocessing, raw EEG-data was directly filtered (dashed lines). Otherwise, identified channels were eliminated and interpolated directly after. Next, raw data was inspected in order to mark artifactual data-segments. Segmen ts marked entailed signal from all channels. Data -filtering conceming line noise at European ranges followed afterwards. An infomax ICA was applied to decompose raw data into single ICs. Non-neural ICs were identified and eliminated with the aid of topographical maps and visually recognizable typical signal characteristics. BVA $=$ Brain Vision Analyzer. IC $=$ Independentcomponent.

responses $\mathrm{R}$ and sum left-hand responses L. The questionnaire was administered online.

\section{German BIS/BAS. A 24-item validated German version of the original BIS/BAS}

questionnaire (Carver \& White, 1994) was implemented here (Strobel, Beauducel, Debener, \& Brocke, 2001). Factorial analyses indicated a two-factor solution consisting of one BISand one BAS factor (Strobel et al., 2001). This is in stark contrast to the proposed four-factor solution of Carver and White (1994) with three BAS factors (fun-seeking, reward responsiveness, and drive) and one BIS factor. Item responses are expressed on 4-point Likert scales $(1=$ strongly disagree, $4=$ strongly agree). The German BAS scale contains items like "I have very few fears compared to my friends" ("Verglichen mit meinen Freunden habe ich sehr wenig Ängste”), “when I get something I want, I get excited and energized" (“wenn ich erreiche, was ich will, bin ich voller Energie und Spannung"), or "if I see something I want, I move on it right away" ("wenn ich eine Chance sehe, etwas Erwünschtes zu bekommen, 
versuche ich sofort mein Glück"). Some exemplary items on the German BIS-scale are "I worry about making mistakes" ("Ich habe Angst, Fehler zu machen"), "criticism or scolding hurts me quite a bit" (“Kritik oder Beschimpfungen verletzen mich ziemlich stark"), or "if I think something unpleasant is about to happen, I usually get pretty worked up" ("wenn ich glaube, dass mir etwas Unangenehmes bevorsteht, bin ich gewöhnlich ziemlich unruhig"). The mean serves as a score for every subscale and was implemented in further analyses. During their conception, analyses indicated good (.81) and acceptable (.78) internal consistency for the BAS and BIS scales, respectively. Split-half reliabilities showed a similar pattern (BAS: .82; BIS: .79; Strobel et al., 2001). The questionnaire was administered online.

German RST-PQ. In the light of correspondence issues between the BIS/BAS scales (Carver \& White, 1994, Strobel et al., 2001) and Gray’s rRST (Gray \& McNaughton, 2000) the RST-PQ was developed (Corr \& Cooper, 2016). The development and validation of a German-translated version followed shortly after and was utilized in the present work (Pugnaghi, Cooper, Ettinger, \& Corr, 2018). The questionnaire consists of 65 items reflecting four rBAS factors: Reward Interest (BASRI), Goal-Drive Persistence (BASGDP), Reward Reactivity (BASRR), and Impulsivity (BASI). BASRI taps openness to new experiences and opportunities that are potentially rewarding. BASGDP may be understood as the capacity to maintain motivation when reward is not immediately available. BASRR relates to the generation and experience of reward. BASI measures the extent to which individuals prefer rash behavior that fosters reinforcement obtainment (Corr, n.d.). The German RST-PQ further comprises one rBIS factor, and one FFFS factor. Participants are asked to respond with a 4point Likert scale to the following question: how accurately does each statement describe you ( $1=$ not at all, $4=$ strongly)? The scales contain items like "I would run quickly if fire alarms in a shopping mall started ringing" ("ich würde schnell losrennen, wenn der Feueralarm in einem Einkaufszentrum anginge"), "when nervous, I sometimes find my thoughts are interrupted" ("wenn ich nervös bin, fällt mir manchmal auf, dass meine Gedanken abreißen”) or "I'm always finding new and interesting things to do" ("ich entdecke immer neue und interessante Dinge, die ich tun kann"). Overall, internal consistencies are acceptable (FFFS: .76; rBIS: .91; reward interest: .80; goal-drive persistence: .82; reward reactivity: .78; impulsivity: .67). The RST-PQ further shows strong convergent and discriminant validity with a variety of established personality measures (Pugnaghi et al., 2018). The questionnaire was administered online.

Karolinska Sleepiness Scale (KSS). Since alpha synchronization is dependent on the wakeful state of subjects (Gillberg, 1990; Kaida et al., 2006), subjective sleepiness during 
recording must be statistically controlled. The KSS is a 10-point Likert scale that consists of the following item with which participants rate their momentary sleepiness: "on a scale of 1 to 10, how sleepy are you feeling right now $(1=$ extremely alert, $3=$ alert, $5=$ neither alert nor sleepy, 7 = sleepy - but no difficulty remaining awake, and $9=$ extremely sleepy - fighting sleep)?” A German translation was implemented for scale administration in the present study.

\section{Statistical Analysis}

All statistical analyses were carried out in RStudio using R 3.6.2 (2019-12-12) and MATLAB 2021a (The MathWorks Inc., Natick, Massachusetts).

The effect of software on the preprocessed EEG-data was examined by comparing differences in every quality measure described in the previous subsection (OHA, THV, CHV, and $\mathrm{RBC}$ ) between automated preprocessing in Automagic and manual preprocessing in BVA. Since none of the quality measures were normally distributed in the sample and between software methods the same participants are compared, two-tailed Wilcoxon signedrank tests for paired samples were carried out to test for differences in the median. We expected quality measures to differ between preprocessing methods albeit not assuming which method would yield higher (or lower) median scores.

Following alpha asymmetry research tradition, average total power from the canonical alpha band $(8-13 \mathrm{~Hz})$ on two mid-frontal (F3, F4), two inferior frontal (F7, F8), two midparietal $(\mathrm{P} 3, \mathrm{P} 4)$, two inferior parietal $(\mathrm{P} 7, \mathrm{P} 8)$, and two occipital $(\mathrm{O} 1, \mathrm{O} 2)$ sites was calculated. Since EEG power values tend to be positively skewed, these measures are usually transformed to its natural log (De Pascalis et al., 2020). The natural log transformation substantially improves the distributional characteristics of the data (Allen et al., 2004). Hence, asymmetry scores were obtained here by subtracting ln-transformed average power of the left electrode from $\ln$-transformed average power on its right homologue (e.g., $\ln (\mathrm{F} 4)-\ln (\mathrm{F} 3))$. Lower asymmetry scores are interpreted as greater right hemispheric resting state cortical activity. First, we investigated whether the degree of asymmetry varied between electrode pairs across eye-conditions and across preprocessing methods. As tested with the ShapiroWilk-test, asymmetry scores were not normally distributed ( $p<0.001$ in most cases). We therefore opted for non-parametric tests since they do not require normal distribution in the data to deliver trustworthy statistics. A Friedman rank-sum test on asymmetry scores was run as an omnibus test to detect any differences in median asymmetry magnitude between electrode pairs. To test for differences in median asymmetry score dependent on 
preprocessing method and eye-condition, we employed the Wilcoxon signed rank test for paired samples. Given that different preprocessing approaches may lead to different results, we expected to observe a main effect for preprocessing method. We did not expect a main effect for the eye-condition as we did not consider that the eye status can change the degree of alpha asymmetry between electrodes.

To inquire whether asymmetry scores deviated from 0 (i.e., no alpha asymmetry) in our sample, we conducted one sample Wilcoxon signed rank tests on asymmetry scores for every electrode pair averaged over conditions, over preprocessing methods as well as for every factor combination. Since there is little evidence as to how asymmetry scores are naturally distributed in a population (for a rare example, see Ocklenburg et al., 2019), we imposed no expectations on the results. Statistical significance in this case is indicative of asymmetric alpha power at a population level. $P$-values are Bonferroni-corrected.

Split- half reliability was calculated for asymmetry scores and single-channel average alpha power using the Spearman-Brown prophecy formula. For this purpose, every preprocessed EEG-dataset was split in two halves of equal length. Specifically, the first $50 \%$ of data was separated from the remaining $50 \%$. The analytical procedure to calculate asymmetry scores was rerun independently for both halves, and for both automatically- and manually preprocessed datasets. We predicted robust split-half reliability for both average alpha power and asymmetry scores.

To quantify the degree of agreement between raters during manual preprocessing and agreement between preprocessing methods, we estimated intraclass correlation coefficients (ICCs). Notice that 'agreement' is to be understood here as the degree of concordance, similarity, or correspondence between two EEG-datasets as a product of the intervention of different raters or software during EEG-preprocessing. Specifically, we will concentrate in agreement of average alpha power and asymmetry scores. This interpretation is best represented mathematically by correlation coefficients. We chose ICCs over other methods, (e.g., product-moment, rank, etc.) for their convenient property of being calculated as the ratio of the variance of groups at higher order levels to the total variance ${ }^{1}$. Large ratios indicate that any two randomly selected observations of the same (also randomly selected) group at a higher order level are more similar to each other than to some other observation of another higher order level group. Similarity within the same group decreases, while similarity to other

\footnotetext{
${ }^{1}$ In our case, groups at higher order levels are raters or preprocessing programs. Total variance is the sum of higher order level-variance and lower-level variance. At the lower-level are independent observations produced by every rater/software. That is, one single asymmetry score/average alpha power value per participant.
} 
higher order level groups increases as ratios decrease (Eid, Gollwitzer, \& Schmitt, 2015). Thus, small coefficients are to be interpreted as high agreement between two preprocessing programs or between two raters. Accordingly, large coefficients indicate a higher similarity between observations of a specific rater/preprocessing program compared to that between observations of different raters/preprocessing programs. We expected ICCs between raters to be similar to ICCs between methods for both asymmetry scores and average alpha power. We further expected both kinds of ICCs to be at least moderate, as this has been observed elsewhere (Hatz et al., 2015).

Furthermore, we calculated correlation coefficients between all personality scales described in the previous section to examine discriminant- and convergent validity. We opted for Spearman rank-correlation coefficients since rho values are robust against normality deviations, which was the case for some of the self-report measures collected here (ShapiroWilk-test, $p<0.001)$.

We further computed bivariate correlations (Spearman's rho) between self-report measures, asymmetry scores, and potential covariates discussed in the previous section to uncover spurious correlations that can potentially confound the relationship between brain asymmetry and personality measures. Hence, in the case of intercorrelations with the variables of interest, these variables were handled as covariates and thus statistically controlled in the subsequent analyses.

Next, partial correlations between all combinations of personality (sub-)scales, asymmetry scores, and covariate were estimated to characterize the degree of relatedness between alpha asymmetry and personality traits without the influence of relevant confounds. Specifically, we calculated single partial correlation matrixes composed of one scale, one asymmetry score, and the covariates. This was done iteratively until one coefficient for every combination of subscales and asymmetry scores and for all four eye-condition/preprocessing method combinations was obtained. Asymmetry scores were extracted at non-frontal electrode pairs to test the hypothesis that asymmetric resting state activity in relation to personality measures is specific for the frontal cortex. Hence, no correlation was expected for posterior electrode pairs. The expected correlational direction and magnitude for every single personality scale was already described in the previous section. We made no assumptions on how different eye-conditions and preprocessing methods might change our predictions. Potential differences across factors are treated here exploratorily. Again, spearman rankcorrelation coefficients were employed to account for non-normality in the data. A two-step 
Bonferroni-correction for multiple comparisons was applied. Specifically, partial correlation coefficients were corrected for multiple comparisons within the partial correlation matrix first, and secondly for multiple comparisons across eye-conditions and preprocessing methods.

We further examined the putative causal relationship between alpha asymmetry and scores of self-report personality scales. For this purpose, we modeled multiple moderation regression analyses. Every model consisted of one personality subscale entered as the independent variable and asymmetry scores stemming from one electrode pair entered as a predictor. We analyzed the moderating role of all three covariates mentioned in the partial correlation analyses on the relationship between asymmetry scores and personality traits. Specifically, we entered one interaction term per covariate as well as three-way- and four-way interactions. Expected relationships between asymmetry scores and personality scales are identical to those for the partial correlation analyses. Assumptions underlying multiple linear regression were checked. Multivariate normality was evaluated visually using quantil-quantilplots ('Q-Q-plots'). Additionally, we implemented a Kolmogorov-Smirnov test to assess normality analytically. The null hypothesis states that residuals do not deviate from normality. The Breusch-Pagan test was used to check whether homoscedasticity is present in the model. The null hypothesis assumes randomly distributed error variance against the alternative that error variance depends on the level of the fitted values or on a linear combination of predictors. We computed variance-inflation factors (VIFs) and used them as a diagnostic for multicollinearity. Following Alin's suggestion (2010), we considered VIFs above 10 as a problematic degree of multiple correlation between one particular predictor and all the other predictors. We further identified single datapoints that have a high impact in the estimation of regression coefficients and fitted values and can change the interpretation of the results dramatically. We defined such influential data points as those which met two criteria. First, datapoints for which their DFFITS lied above the arbitrary value of 1 . Second, datapoints whose cook's distance surpassed a critical $F$-value. The respective $F$-value threshold was computed by setting $\alpha$ at 0.50 . Having identified influential datapoints, we opted for a sensitivity analysis, rather than excluding them. That is, we characterized the impact of influential values on model-fitting by reporting model parameters and $p$-values with- and without said data points (see 'Results'). Again, we expected that variance in personality scores would be predicted by frontal electrode pairs rather than by posterior ones. Similar to partial correlation analyses, we merely explored differences between regression models across preprocessing methods and eye-conditions without making any predictions. $P$-values obtained from every regression model were Bonferroni-corrected for multiple testing. 
For all correlational, non-parametric, and regression analyses, observations surpassing three standard deviations of their corresponding variable were considered implausible and therefore excluded beforehand. Analyses regarding data quality are spared from this criterion since every quality measure examined is intended to be sensitive to voltage deflections of any magnitude. Data exclusion would distort the degree to which both preprocessing methods identified and eliminated artifactual portions of EEG-data. For all analyses, the (not corrected) statistical significance threshold was set at .05 .

\section{Results}

In the following, results are separated thematically in different subsections. Accordingly, we present our findings concerning quality measures, questionnaires, alpha power, alpha asymmetry, partial correlations, and moderated regression models.

\section{Quality measures}

Since the distribution of quality measures drastically deviate from normality, the mean and standard deviation are not informative descriptive statistics. Moreover, most of quality measures are shifted toward zero and few outliers expand the data range so greatly such that the median and ranges are not suitable statistical values either. For these reasons, descriptive statistics are omitted in this subsection.

A two-tailed Wilcoxon signed-rank test conducted on EC-datasets indicated that scores for OHAs were significantly smaller in datasets preprocessed automatically using Automagic than in manually using BVA sets, $Z=4.061, p<0.001, r=0.592$. Similarly, scores for THVs after automated preprocessing were smaller than those obtained after manual preprocessing, $Z=5.409, p<0.001, r=0.789$. CHV scores after automated preprocessing were also smaller than CHV scores after manual preprocessing, $Z=3.281, p=0.001, r=$ 0.479. RBC-scores observed after automated preprocessing were greater than RBC-scores using the manual approach, $Z=5.780, p<0.001, r=0.843$.

Analyses conducted on EO-datasets indicated that scores for OHAs were significantly smaller in Automagic datasets than in BVA datasets as well, $Z=4.049, p<0.001, r=0.604$. THV scores using automated preprocessing were smaller than those obtained after manual preprocessing, $Z=4.950, p<0.001, r=0.738$. CHV scores after implementing automated 
preprocessing were also smaller than $\mathrm{CHV}$ scores stemming from the manual approach, $\mathrm{Z}=$ 2.913, $p=0.004, r=0.434$. Automated preprocessing produced RBC scores greater than RBC scores produced by manual preprocessing, $Z=5.780, p=0.009, r=0.390$. Figure 3 shows a comparison between distributions of all quality measures separated by eye-condition and preprocessing method.

\section{Questionnaires}

LQs showed a J-distribution in the sample (Median $=88.889 ;$ min. $=-100$, max. $=$ 100), showing that right-handers represent the majority of participants in the sample. Sleepiness averaged to 3.872 points $(S D=1.596)$ on the KSS. Following Blanz's interpretation guidelines (2015), the classic BIS and BAS scales showed good $(\alpha=0.84)$ and questionable $(\alpha=0.6)$ internal consistencies, respectively. On average, participants reached a score of $3.213(S D=0.288)$ on the BAS scale and a score of $2.764(S D=0.312)$ on the BIS scale. In contrast, rBIS showed an excellent internal consistency $(\alpha=0.92)$ and rBAS' cronbach's alpha was 0.86 . Here, we registered a mean rBIS score of 52.404 with a standard deviation of 13.013 and a mean rBAS score of 92.717 with a standard deviation of 10.901 . The rBAS subscales, BAS GDP ( $\alpha=0.83)$, BAS RI $(\alpha=0.79)$, BAS RR $(\alpha=0.79)$, and BAS $\mathrm{I}(\alpha=0.55)$ varied in their reliability greatly. On average, participants reached a BAS GDP score of 22.847 ( $S D=3.663)$, a BAS RI score of $20.298(S D=4.027)$, a BAS RR score of $30.404(S D=5.203)$, and a BAS I score of $18.745(S D=3.632)$. Lastly, FFFS's internal consistency was acceptable $(\alpha=0.70)$. Participants scored on average 22.106 points on the FFFS-scale $(S D=5.087)$

Classic BAS showed a strong relationship to the RST-PQs subscale BAS GDP, $\rho=$ $0.509, p=0.01$, and to the $\mathrm{rBAS}, \rho=0.476, p=0.02$. As expected, rBAS correlated strongly with the subscales rBAS GDP, rBAS RR and rBAS RI, $(0.675<\rho<0.772, p<0.01)$, except for rBAS I, which did not correlate significantly, $\rho=0.461, p=0.09$. rBAS GDP also showed a moderate monotonic relationship to $\mathrm{rBAS} \mathrm{RI}, \rho=0.463, p<0.01$, and to $\mathrm{rBAS} \mathrm{RR}, \rho=$ $0.462, p=0.01$. Table 1 presents a summary of bivariate correlations between all self-report scales, with $p$-values corrected for multiple comparisons.

\section{Alpha Power and Alpha Asymmetry}

Split-half reliabilities for the average alpha power per site obtained from BVApreprocessed EEG-data were mostly excellent during the EO-condition $(M=0.956, S D=$ 
RBC

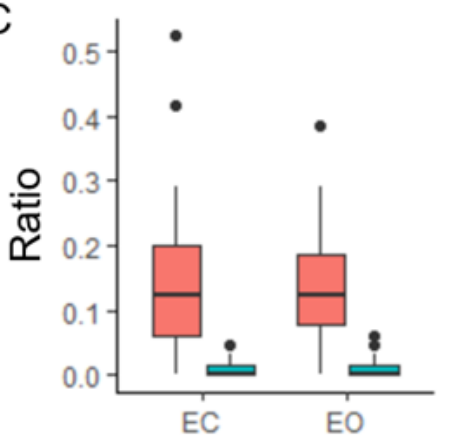

\section{$\mathrm{CHV}$}

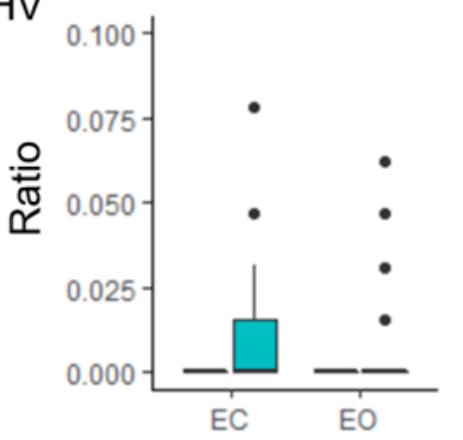

THV

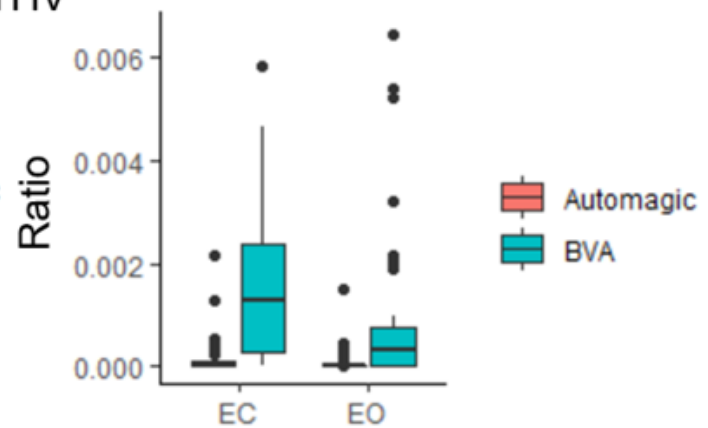

$\mathrm{OHA}$

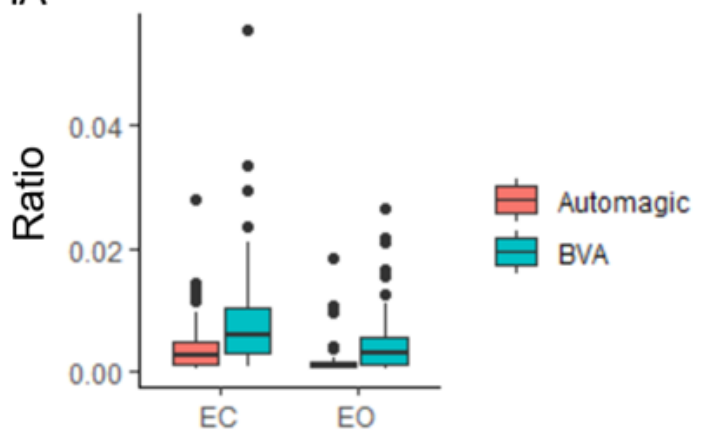

Fig.3. Boxplots of quality measures. Scores are separated by eye condition ( $\mathrm{x}$-axes) and preprocessing method (Automagic: red; BVA: blue). Y-axes show the ratio of quality scores that surpassed the arbitrary magnitude threshold of $30 \mu \mathrm{V}$ and the standard deviation of $15 \mu \mathrm{V}$ for measures based on the magnitude of the amplitude of the EEG-signal, i.e, OHA, CHV, and THV. Notice that some measures are heavily skewed toward zero. Hence, some boxes are not visible. Some outliers were omitted for display purposes. $\mathrm{RBC}=$ ratio of bad channels; timepoints of high variance; $\mathrm{CHV}=$ channels of high variance; $\mathrm{OHA}=$ overall high amplitude. $\mathrm{THV}=$ timepoints of high variance.

0.058; $\min .=0.794, \max .=0.990)$. In stark contrast to this, reliabilities for the EC-condition varied greatly $(M=0.483, S D=0.374$; $\min .=0.067$, max. $=0.964)$. A similar pattern was observed for asymmetry scores extracted from BVA-data. For the EO-condition, alpha asymmetry scores yielded reliabilities ranging from 0.937 to $0.982(M=0.943, S D=0.029)$. Estimates of split-half reliability of alpha asymmetry scores ranged from 0.631 to $0.967(M=$ $0.853, S D=0.134)$ during the EC-condition.

Split-half reliabilities observed for alpha power obtained when using an automated approach to EEG preprocessing were consistently excellent for both EC- $(M=0.959, S D=$ 0.011 ; $\min .=0.940, \max .=0.975)$ and EO-conditions- $(M=0.988, S D=0.005 ; \min .=0.980$, max. $=0.989)$. The same pattern holds for reliabilities estimated for asymmetry scores. Both EC $-(M=0.958, S D=0.025 ; \min .=0.926$, max. $=0.983)$ and EO-conditions $(M=0.965, S D$ $=0.016 ;$ min. $=0.952$, max. $=0.997)$ showed split-half reliabilities above 0.95 . Table 2 summarizes split-half reliabilities found for average power and asymmetry scores for every electrode, eye-condition, and preprocessing method. 
Table 1

Bivariate Spearman Correlation Coefficients between Self-Report Measures of Personality

\begin{tabular}{llllllllll}
\hline Scale & BAS & BIS & rBAS GDP & rBAS RI & rBAS RR & rBAS I & rBIS & FFFS & rBAS \\
\hline BAS & 1.000 & & & & & & & & \\
BIS & 0.120 & 1.000 & & & & & & & \\
rBAS GDP & $0.509^{*}$ & 0.104 & 1.000 & & & & & & \\
rBAS RI & 0.388 & 0.049 & $0.463^{* *}$ & 1.000 & & & & & \\
rBAS RR & 0.415 & 0.285 & $0.462^{*}$ & 0.316 & 1.000 & & & & \\
rBAS I & -0.075 & -0.086 & -0.096 & 0.247 & 0.216 & 1.000 & & & \\
rBIS & -0.400 & 0.346 & -0.146 & -0.261 & -0.050 & 0.222 & 1.000 & & \\
FFFS & 0.202 & 0.143 & 0.246 & 0.103 & 0.335 & 0.127 & 0.141 & 1.000 & \\
rBAS & $0.476^{*}$ & 0.192 & $0.675^{* *}$ & $0.740^{* *}$ & $0.772^{* *}$ & 0.461 & -0.069 & 0.273 & 1.000 \\
\hline
\end{tabular}

BAS = Behavioral Activation System, BIS = Behavioral Inhibition System, FFFS = Fight-Flight-Freeze-System, GDP = Goal-Drive Persistence, $\mathrm{RI}=$ Reward Interest, $\mathrm{RR}=$ Reward Reactivity, $\mathrm{I}=$ Impulsivity, $\mathrm{r}=$ revised, ${ }^{*} p<0.05$, $* * p<$ $0.01, * * * p<0.001 . P$-values are Bonferroni-corrected for multiple comparisons.

Agreement between preprocessing methods varied greatly, as shown by ICCs computed for alpha power on single electrodes for EC-recordings $(M=0.415, S D=0.329$; $\min .=0.029, \max .=0.739)$. ICCs for frontal cites were especially low. For EO-recordings, agreement was more balanced $(M=0.687, S D=0.090 ; \min .=0.456, \max .=0.780)$. Interrater reliability ranged from 0.019 to 0.992 for EC-recordings $(M=0.577, S D=0.482)$. Again, ICCs for frontal and posterior electrodes were extremely low and high, respectively. Interestingly, coefficients between human raters were almost always exceptionally high for EO-recordings $(M=0.978, S D=0.057$; $\min .=0.815, \max .=0.999)$.

ICCs for alpha asymmetry scores between raters ranged from -0.229 to $0.434(M=-$ $0.036, S D=0.323)$, and from 0.831 to $0.970(M=0.903, S D=0.061)$ for EO- and ECdatasets, respectively. For cross-method agreement, ICCs ranged from -0.113 to $0.481(M=$ $0.091, S D=0.248)$ during EO-recordings and from -0.080 to $0.775(M=0.499, S D=0.348)$ during EC-recordings. Table 3 displays ICCs for both power and asymmetry scores across preprocessing methods and conditions.

A two-sided Friedman test on asymmetry scores found a main effect for electrode pair, $\chi^{2}(4)=18.009, p=0.001$. Post-hoc tests revealed a significant difference in the score distribution between electrode pairs P7/P8 $($ Median $=0.913$, min. $=-3.882$, max.$=4.429)$ and F7/F8 (Median $=-0.195$, min. $=-1.926$, max. $=3.731) . \mathrm{P} 7 / \mathrm{P} 8$ had a greater median score than F7/F8, $p<0.05$. Both P7/P8 (Median $=0.913$, $\min .=-3.882, \max .=4.429)$ and P3/P4 $($ Median $=0.823, \min .=-2.602, \max .=3.055)$ differed in their score distributions from that 
Table 2

Split-Half Reliabilites (Spearman-Brown) for AverageAlpha Power (8-13 Hz) and Asymmetry Scores

$\begin{array}{lllllllllll}\begin{array}{l}\text { Alpha } \\ \text { Power }\end{array} & \text { F3 } & \text { F4 } & \text { F7 } & \text { F8 } & \text { P3 } & \text { P4 } & \text { P7 } & \text { P8 } & \text { O1 } & \text { O2 } \\ \text { BVA } & & & & & & & & & & \\ & & & & & & & & & & \\ \text { EC } & 0.134 & 0.067 & 0.237 & 0.174 & 0.088 & 0.964 & 0.782 & 0.956 & 0.761 & 0.663 \\ \text { EO } & 0.978 & 0.978 & 0.968 & 0.794 & 0.974 & 0.979 & 0.962 & 0.990 & 0.960 & 0.977\end{array}$

Automagic

$\begin{array}{lllllllllll}\text { EC } & 0.965 & 0.960 & 0.961 & 0.946 & 0.953 & 0.965 & 0.974 & 0.975 & 0.942 & 0.950 \\ \text { EO } & 0.993 & 0.986 & 0.987 & 0.980 & 0.987 & 0.983 & 0.989 & 0.997 & 0.987 & 0.988\end{array}$

Asymmetry

Scores

F3/F4 $\quad$ F7/F8 P3/P4 P7/P8 O1/O2

BVA

$\begin{array}{lccccc}\text { EC } & 0.967 & 0.828 & 0.631 & 0.930 & 0.911 \\ \text { EO } & 0.982 & -- & 0.937 & 0.911 & 0.940\end{array}$

Automagic

$\begin{array}{llllll}\text { EC } & 0.926 & 0.983 & 0.941 & 0.983 & 0.957\end{array}$

$\begin{array}{llllll}\text { EO } & 0.981 & 0.984 & 0.959 & 0.952 & 0.949\end{array}$

Split-half reliabilities concerning Asymmetry scores and average canonical alpha band power $(8-13 \mathrm{~Hz})$ are displayed for every electrode and electrode pair divided by eye-condition and preprocessing method. Each recording was split in time into two equal halves. One half representing the first $50 \%$ of datapoints, and one half representing the remaining 50\%. For each half, alpha power and asymmetry scores were computed. We computed reliability-coefficients using the Spearman-Brown correction. An interpretable estimate for asymmetry scores obtained from the F7/F8-pair during EO-recordings could not be computed and is therefore omitted. BVA = Brain Vision Analyzer, EC = Eyes Closed, EO = Eyes Open.

of F3/F4's (Median $=-0.064$, min. $=-2.297$, max. $=3.699), p<0.05$. No other significant differences between electrode pairs were found. The main effect of preprocessing method, $\chi^{2}(1)=0.191, p=0.662$, as well as the main effect of eye-condition, $\chi^{2}(1)=1.043, p=0.307$, were not significant.

Wilcoxon rank-sum tests revealed right-asymmetric alpha power in the electrode pair P7/P8 (Median $=0.413$, min. $=-1.810$, ax.$=1.589), Z=-3.949, p<0.001, r=0.576$, for scores computed from EC-datasets preprocessed automatically in Automagic. Asymmetry scores at P7/P8 $($ Median $=0.221, \min .=-0.885, \max .=1.381)$ were also different from 0 for automatically preprocessed EO-datasets, skewed towards right asymmetric alpha power, $Z=$ 3.320, $p<0.001, r=0.495$. Scores from EC-datasets preprocessed by hand at P7/P8 (Median 
Table 3

Intraclass Correlation Coefficients for Alpha Power at Single Electrodes and Asymmetry Scores

\begin{tabular}{lllllllllll}
\hline Alpha Power & F3 & F4 & F7 & F8 & P3 & P4 & P7 & P8 & O1 & O2 \\
BVA-Automagic & & & & & & & & & & \\
EC & & & & & & & & & & \\
EO & 0.046 & 0.051 & 0.051 & 0.029 & 0.441 & 0.672 & 0.711 & 0.739 & 0.700 & 0.705 \\
& 0.692 & 0.670 & 0.673 & 0.456 & 0.672 & 0.707 & 0.743 & 0.780 & 0.725 & 0.756
\end{tabular}

Raters

$\begin{array}{lllllllllll}\text { EC } & 0.019 & 0.023 & 0.023 & 0.012 & 0.877 & 0.885 & 0.990 & 0.991 & 0.992 & 0.959 \\ \text { EO } & 0.999 & 0.999 & 0.986 & 0.992 & >0.999 & >0.999 & 0.990 & >0.999 & 0.815 & 0.998\end{array}$

$\begin{array}{llllll}\begin{array}{l}\text { Asymmetry } \\ \text { Scores }\end{array} & \text { F3/F4 } & \text { F7/F8 } & \text { P3/P4 } & \text { P7/P8 } & \text { O1/O2 } \\ \text { BVA-Automagic } & & & & & \\ \text { EC } & & & & & \\ \text { EO } & -0.080 & 0.490 & 0.545 & 0.775 & 0.763 \\ & -0.016 & 0.188 & -0.113 & 0.481 & -0.085\end{array}$

Raters

\begin{tabular}{llllll} 
EC & 0.957 & 0.856 & 0.899 & 0.970 & 0.831 \\
EO & 0.013 & 0.026 & -0.229 & 0.434 & -0.426 \\
\hline
\end{tabular}

Coefficients represent agreement between two entities. Values under the 'BVA-Automagic' section represent agreement between both preprocessing methods. Values under 'Raters' represent measures of interrater reliability. BVA = Brain Vision Analyzer, EC = Eyes Closed, EO = Eyes Open. See main text for a discussion of negative ICCs.

$=0.332$, $\min .=-1.066, \max .=1.626)$ were also asymmetric, $Z=-3.671, p<0.001, r=0.536$, implying rightward alpha power. Finally, both F3/F4 (Median $=-0.203$, min. $=-0.794$, max. $=$ 2.687) and P3/P4 (Median $=0.557, \min .=-0.860, \max .=1.650)$ pairs deviated significantly from zero for BVA-preprocessed EO-datasets $(Z=-5.019, p<0.001, r=0.748$ and $Z=$ 4.414, $p<0.001, r=0.658$, respectively). While F3/F4 showed leftward alpha power, P3/P4's distribution was skewed to more positive asymmetry scores, implying right frontal alpha power. Figure 4 displays asymmetry score distribution for every electrode pair separated by eye-condition and preprocessing method.

\section{Partial Correlation Analysis}

Bivariate correlations between putative covariates, asymmetry scores and self-report measures were found. Specifically, O1/O2-asymmetry scores were positively correlated to sleepiness, $\rho=0.343, p=0.024$, when EC-datasets were preprocessed manually. Both handedness, $\rho=0.308, p=0.047$, and sleepiness, $\rho=0.305, p=0.050$, correlated positively with asymmetry scores computed from electrodes F7 and F8 after manual preprocessing of 


\section{A Asymmetry Scores Eyes Closed}

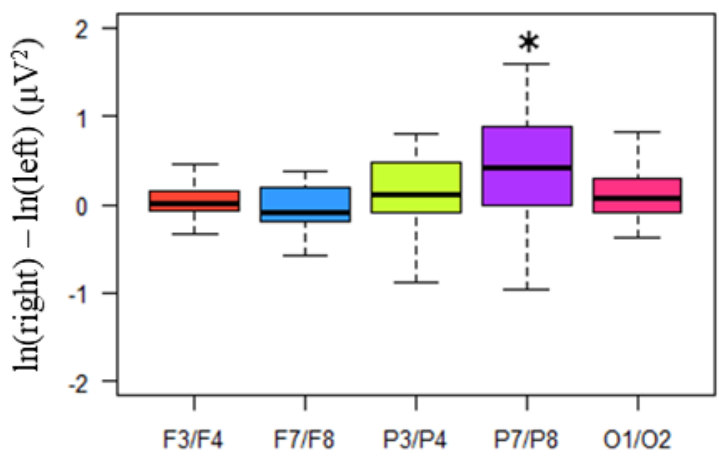

B Asymmetry Scores Eyes Closed

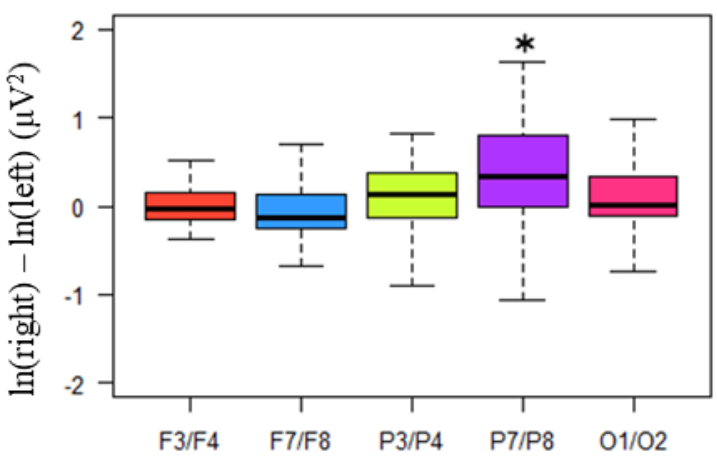

Asymmetry Scores Eyes Open

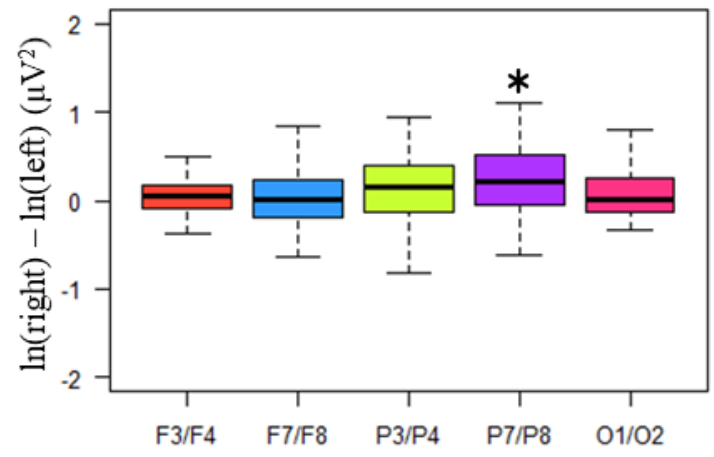

Asymmetry Scores Eyes Open

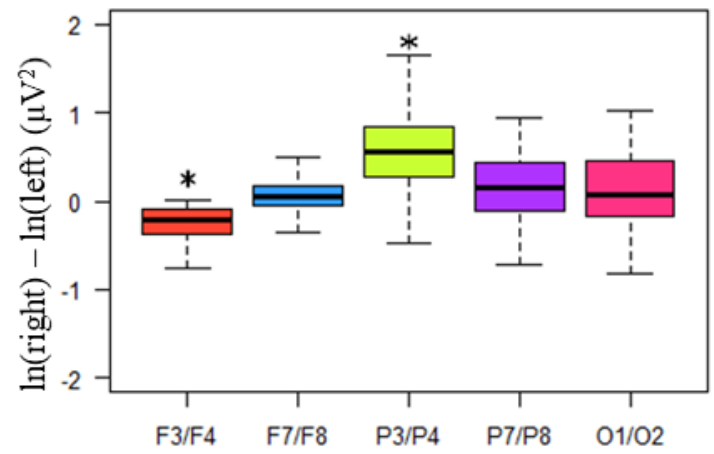

Fig.4. Boxplots of asymmetry scores for every electrode pair in $\mu \mathrm{V}^{2}$. Scores are obtained by subtracting the ln-transformed left total alpha power from its right homologue. Moreover, the total alpha power used is the average of the whole recording, in consideration of the canonical alpha band $(8 \mathrm{~Hz}-13 \mathrm{~Hz})$. A) Boxplots for asymmetry scores obtained using Automagic. Left: Eyes Closed; right: eyes open. B) Boxplots for asymmetry scores obtained using Brain Vision Analyzer 2.0. Left: eyes closed; right: eyes open. Outliers are omitted for optimal display. Asterisks indicate a significant deviation from $0(p<0.05$, Bonferronicorrected).

EO-datasets. Furthermore, sleepiness was negatively associated with classic BAS, $\rho=-0.350$, $p=0.0160$. Any other association to covariates turned out statistically insignificant (all $p$ 's $>$ 0.05). Supplementary figures $1,2,3$ and 4 in the appendix offer heatmaps of bivariate correlations between asymmetry scores, personality scales, and covariates for every $\mathrm{method} /$ condition combination. Overall, the pattern of intercorrelations found support for the statistical control of covariates in order to interpret personality asymmetries confound-free in the next step.

After correcting for multiple testing, no partial correlation between personality questionnaires and asymmetry scores reached statistical significance (all $p$ 's $>0.05$ ). Table 4 provides an overview of every partial correlation coefficient obtained. For a comprehensive view of test-statistics concerning partial correlations, see supplementary tables $1,2,3$, and 4 in the Appendix. 
Table 4

Partial Correlations (Spearman rank-correlation coefficients) between Self-Report Measures of Personality and Asymmetry Scores controlled for Handedness, Gender and Sleepiness

\begin{tabular}{|c|c|c|c|c|c|c|c|c|c|}
\hline Scale & BAS & BIS & rBAS & rBASGDP & rBASRI & rBASRR & rBASI & rBIS & FFFS \\
\hline \multicolumn{10}{|c|}{$\begin{array}{l}\text { Automagic } \\
\text { EC }\end{array}$} \\
\hline $\mathrm{F} 3 / \mathrm{F} 4$ & 0.032 & -0.102 & 0.065 & 0.372 & -0.154 & -0.031 & 0.013 & 0.276 & 0.052 \\
\hline F7/F8 & 0.065 & -0.015 & 0.014 & 0.241 & -0.311 & 0.127 & -0.019 & 0.270 & 0.017 \\
\hline $\mathrm{P} 3 / \mathrm{P} 4$ & -0.053 & -0.129 & -0.124 & -0.338 & 0.136 & -0.203 & 0.263 & -0.137 & 0.008 \\
\hline $\mathrm{P} 7 / \mathrm{P} 8$ & 0.285 & -0.018 & 0.112 & -0.07 & 0.268 & 0.012 & 0.149 & -0.277 & -0.078 \\
\hline \multicolumn{10}{|c|}{$\begin{array}{l}\text { Automagic } \\
\text { EO }\end{array}$} \\
\hline $\mathrm{F} 3 / \mathrm{F} 4$ & 0.091 & -0.05 & -0.261 & -0.130 & -0.218 & -0.134 & -0.249 & 0.063 & -0.223 \\
\hline F7/F8 & 0.217 & -0.185 & 0.006 & 0.166 & -0.032 & -0.077 & 0.048 & 0.040 & -0.222 \\
\hline P3/P4 & 0.013 & 0.149 & 0.124 & -0.090 & 0.159 & 0.091 & 0.190 & 0.018 & 0.155 \\
\hline $\mathrm{P} 7 / \mathrm{P} 8$ & 0.105 & -0.031 & 0.186 & 0.084 & 0.082 & 0.115 & 0.212 & -0.193 & 0.211 \\
\hline $\mathrm{O} 1 / \mathrm{O} 2$ & -0.035 & 0.059 & 0.020 & 0.018 & -0.083 & -0.007 & 0.017 & 0.127 & -0.244 \\
\hline \multicolumn{10}{|c|}{ BVA EC } \\
\hline F3/F4 & -0.05 & -0.008 & -0.377 & -0.106 & -0.296 & -0.248 & -0.289 & 0.279 & 0.106 \\
\hline F7/F8 & 0.084 & 0.174 & -0.035 & 0.103 & -0.274 & 0.052 & -0.034 & 0.189 & -0.031 \\
\hline $\mathrm{P} 3 / \mathrm{P} 4$ & -0.089 & 0.001 & -0.105 & -0.366 & 0.082 & -0.161 & 0.355 & 0.034 & 0.161 \\
\hline $\mathrm{P} 7 / \mathrm{P} 8$ & 0.127 & -0.08 & 0.170 & 0.006 & 0.231 & 0.127 & 0.294 & -0.267 & 0.060 \\
\hline $\mathrm{O} 1 / \mathrm{O} 2$ & -0.066 & -0.049 & 0.036 & 0.009 & -0.049 & 0.094 & 0.078 & 0.038 & -0.162 \\
\hline \multicolumn{10}{|c|}{ BVA EO } \\
\hline $\mathrm{F} 3 / \mathrm{F} 4$ & 0.261 & -0.098 & 0.188 & 0.036 & 0.241 & 0.068 & 0.120 & 0.059 & -0.102 \\
\hline F7/F8 & 0.076 & -0.142 & -0.308 & -0.127 & -0.194 & -0.215 & -0.233 & 0.009 & -0.166 \\
\hline P3/P4 & -0.475 & -0.008 & -0.211 & -0.356 & -0.126 & -0.287 & 0.275 & 0.237 & -0.145 \\
\hline P7/P8 & -0.116 & 0.190 & 0.132 & -0.139 & 0.172 & 0.169 & 0.224 & 0.113 & 0.153 \\
\hline $\mathrm{O} 1 / \mathrm{O} 2$ & -0.16 & -0.447 & -0.13 & -0.047 & 0.111 & -0.119 & 0.008 & -0.138 & 0.002 \\
\hline
\end{tabular}

$p$-values were obtained by applying a Bonferroni-correction for multiple comparisons within the partial correlation matrix and for all comparisons across electrode pairs, conditions, and preprocessing methods (see main text). No coefficient's $p$-value reached statistical significance $\left(\alpha_{\mathrm{adj}}=0.0003\right)$. BAS $=$ Behavioral Activation System, $\mathrm{BIS}=$ Behavioral Inhibition System, BVA = Brain Vision Analyzer, EC = eyes closed, EO = eyes open, FFFS = Fight-FlightFreeze-System, GDP = Goal-Drive Persistence, RI = Reward Interest, $\mathrm{RR}=$ Reward Reactivity, $\mathrm{I}=$ Impulsivity, $\mathrm{r}=$ revised.

\section{Moderated Regression Analysis}

For the sake of the reader's clarity, only results for which either the model variant with influential values or the variant without them yielded statistically significant results after multiple-comparison correction are reported in this section. Additionally, only models 
fulfilling every assumption underlying linear regression are discussed. Due to the small critical $\alpha$-value after correction (0.000277) the following $p$-values are presented with a rounding to the sixth decimal place.

For manually preprocessed EC-datasets, rBIS values increased when F3/F4 asymmetry scores did, $\beta=42.155, p=0.000233$. Sleepiness moderated this relationship. As sleepiness increases, so does the estimated coefficient for F3/F4 asymmetry scores, $\beta=42.806, p=$ 0.000053. As the Johnson-Neyman plot in Figure 6 shows, this effect applies only for KSS values greater than $3, p<0.05$. The model's fit, however, merely showed a tendency towards significance after correcting for multiple comparisons, $R^{2}{ }_{a d j}=0.456, F(8,29)=4.877, p=$ 0.000698. Furthermore, these observations do not hold without the exclusion of influential values, $R_{a d j}^{2}=0.050, F(8,34)=1.278, p=0.287200$ (Table 5). Figure 5 displays the interplay between rBIS scores, manually preprocessed EC-asymmetry scores (F3/F4), and centered KSS values. Every other multiple regression model fitted either yielded non-significant estimates and goodness of fit, or at least one assumption underlying linear regression was violated and were therefore unreliable for interpretation.

\section{Discussion}

The present work probed the impact of two different preprocessing methods on the analysis of resting-state EEG-data. Specifically, we compared the influence of a traditional preprocessing workflow implemented in Brain Vision Analyzer 2.0. including visual detection and rejection of artifactual data-segments and ICs based on human judgement to that of the fully standardized toolbox 'Automagic' (Pedroni et al., 2019). We first examined changes in data quality using measures that assess the quality of EEG-datasets using criteria based on signal amplitude. Next, we explored alpha power reliability, agreement between human raters and agreement between software on alpha power. Furthermore, we calculated asymmetry scores to operationalize alpha power asymmetry between several homologous pairs of electrodes. Reliability and agreement calculations for asymmetry scores followed the same way as calculations for average alpha power on single electrodes. Finally, we were interested in finding empirical evidence for well-documented associations between alpha power asymmetries at rest and personality traits based on Gray's RST and revised versions thereof (Corr \& McNaughton, 2008; Gray 1982; Gray \& McNaughton, 2000). Instead of merely replicating effects concerning frontal alpha asymmetry, we decided to address 
Table 5

Moderated Regression Models for manually preprocessed EEG-data (EC). F3/F4Asymmetry Scores as Predictors of rBIS Scores.

\begin{tabular}{|c|c|c|c|c|}
\hline & $\boldsymbol{\beta}$ & $S E$ & $t$ & $p$ \\
\hline \multicolumn{5}{|l|}{ Model $1(n=43)$} \\
\hline Intercept & 52.389 & 2.047 & 25.595 & $<2 \mathrm{e}-16^{* * *}$ \\
\hline $\mathrm{F} 3 / \mathrm{F} 4$ & 18.769 & 10.582 & 1.774 & 0.085100 \\
\hline $\mathrm{F} 3 / \mathrm{F} 4 * \mathrm{KSSc}$ & 16.119 & 6.455 & 2.497 & 0.017500 \\
\hline F3/F4*LQ & -0.047 & 0.132 & -0.354 & 0.725500 \\
\hline F3/F4*Gender & -11.406 & 22.627 & -0.504 & 0.617500 \\
\hline F3/F4*KSSc*Gender & -7.990 & 13.504 & -0.592 & 0.558000 \\
\hline F3/F4*LQ*Gender & -0.068 & 0.276 & -0.245 & 0.807900 \\
\hline F3/F4*KSSc*LQ & -0.185 & 0.081 & -2.292 & 0.028200 \\
\hline F3/F4*KSSc*LQ*Gender & 0.227 & 0.145 & 1.562 & 0.127500 \\
\hline \multicolumn{5}{|l|}{ Model $2(n=38)$} \\
\hline Intercept & 49.340 & 1.619 & 30.478 & $<2 \mathrm{e}-16 * * *$ \\
\hline $\mathrm{F} 3 / \mathrm{F} 4$ & 42.155 & 10.041 & 4.198 & $0.000233 * * *$ \\
\hline $\mathrm{F} 3 / \mathrm{F} 4 * \mathrm{KSSc}$ & 27.806 & 5.875 & 4.733 & $5.33 \mathrm{e}-05 * * *$ \\
\hline $\mathrm{F} 3 / \mathrm{F} 4 * \mathrm{LQ}$ & 0.1978 & 0.113 & 1.753 & 0.090173 \\
\hline F3/F4*Gender & -9.919 & 268.288 & -0.037 & 0.970760 \\
\hline $\mathrm{F} 3 / \mathrm{F} 4 * \mathrm{KSSc}^{*}$ Gender & -38.675 & 154.522 & -0.250 & 0.804128 \\
\hline F3/F4*LQ*Gender & -0.665 & 2.991 & -0.222 & 0.825512 \\
\hline $\mathrm{F} 3 / \mathrm{F} 4 * \mathrm{KSSc}^{*} \mathrm{LQ}$ & 0.017 & 0.0938 & 0.183 & 0.856079 \\
\hline $\mathrm{F} 3 / \mathrm{F} 4 * \mathrm{KSSc}^{*} \mathrm{LQ} *$ Gender & 0.1742 & 0.1743 & 0.116 & 0.908351 \\
\hline
\end{tabular}

Asymmetry scores computed for electrode pair F3/F4 were entered as predictor and rBIS scores as criterium. Gender, Handedness and Sleepiness are modeled to moderate this relationship. To overcome multicollinearity issues, KSS-scores were centered ("KSSc"). Model 1 was fit including extreme values. Model 1 does not fit the data $F(8,34)=1.278, p=0.287200$. Model 2 is obtained by excluding extreme values. Model 2 shows a numerical tendency to predict $45.6 \%$ of the variance, $F(8,29)=4.877, p=0.0006982$. $P$-values are Bonferroni-corrected for multiple comparisons (180) and are therefore displayed up to the sixth decimal place. $\mathrm{EC}=$ eyes closed, $\mathrm{KSS}=$ Karolinska Sleepiness Scale, $\mathrm{LQ}=$ laterality quotient,$* * * p<0.000278$.

empirical inconsistencies by additionally employing self-report measures featured in the RSTPQ (Corr \& Cooper, 2016) that putatively tap more accurately on theoretical biopsychological systems of personality proposed by RST than the most popular self-report measures of motivational personality, the BIS/BAS-scales from Carver \& White (1994). In the following, we will address each analysis undertaken in separate subsections.

\section{Questionnaires}

Overall, correlational analyses performed on self-report measures of personality confirmed our predictions. Consistent with theoretical inquiries about the relationship between the FFFS and rBIS, a weak but not significant association between the two was observed here, showing discriminant validity between constructs. This supports the idea that 


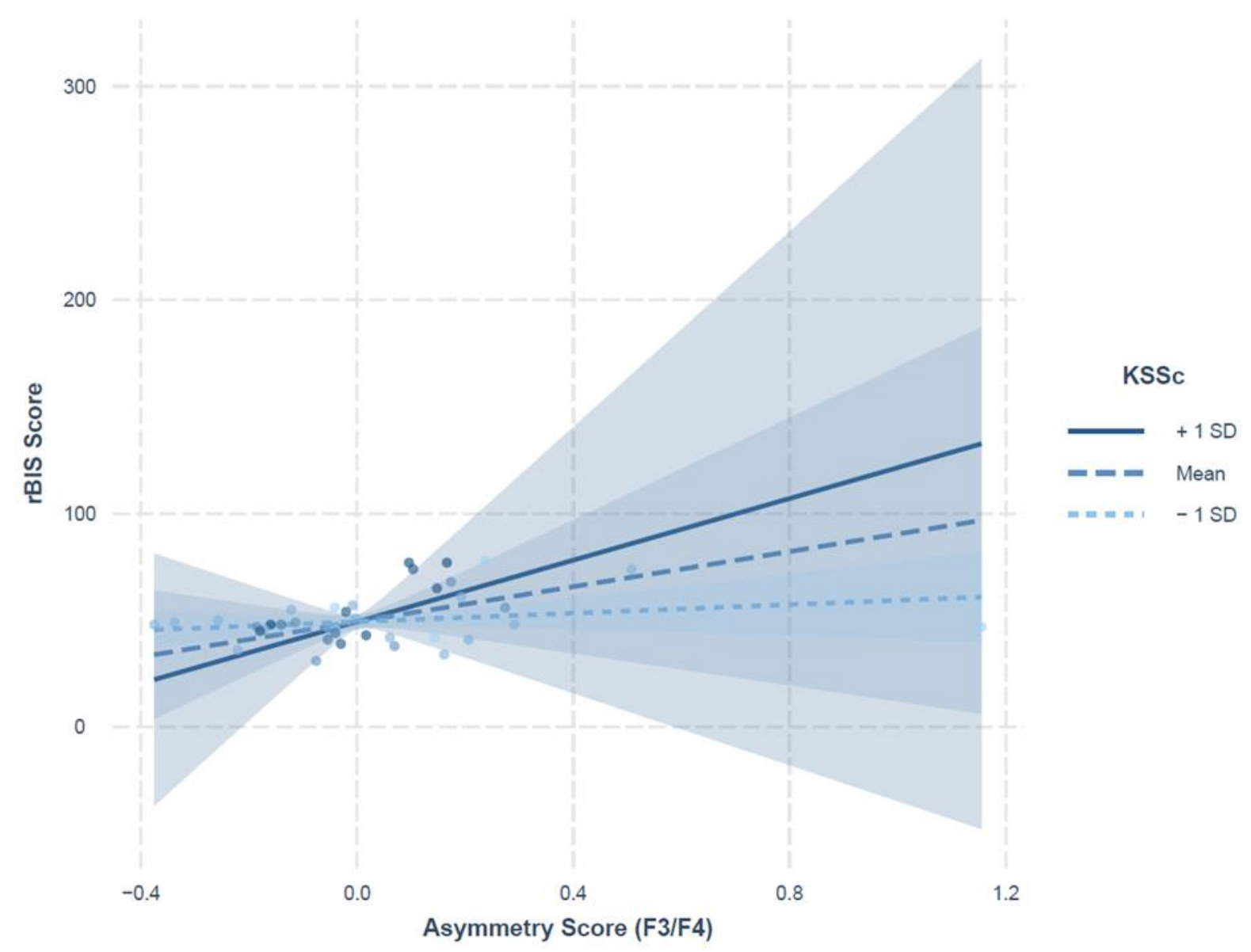

Fig.5. Interaction plot for the linear relationship between rBIS Scores (criterium) and asymmetry scores extracted from the electrode pair F3/F4 (predictor), where the level of sleepiness changes the estimated slope (moderator). The model was fitted with alpha power obtained from manually preprocessed EC-recordings and does not contain extreme values (see model 2 in Table 5 and main text). EEG-data was manually preprocessed. Fitted regression lines are displayed for the mean on the moderator variable, as well as for 1 standard deviation around the mean. Notice that KSS values are mean-centered (hence, "KSSc"). EC = eyes closed, KSS = Karolinska Sleepiness Scale, rBIS = revised Behavioral Inhibition System .

while both systems are frequently concurrently activated, they are nonetheless separate systems (Corr \& McNaughton, 2008; Gable et al., 2018). Indeed, FFFS-related processes have been ascribed to activity in subcortical regions such as the amygdala and the insula in fMRI studies (Dilger et al., 2003; Sabatinelli, Bradley, Fitzsimmons, \& Lang, 2005; Schienle et al., 2002). In contrasts, rBIS functionality is influenced by right-hemispheric frontal cortical regions such as the inferior frontal cortex, the dorsolateral frontal cortex, and the cingulate gyrus (Gable et al., 2018).

Strong correlations between rBAS and its subscales are unsurprising since rBASscores are the sum of its subscales. Even though an aggregate rBAS-scale is not part of the factor solution of the German RST-PQ (Pugnaghi et al., 2018), we included one nonetheless to facilitate comparability with the classic BAS scale in all analyses. We argue that a superordinate rBAS scale is justified given that the subscales showed a high degree of intercorrelation with each other. It is worth noting that rBAS I did not show any significant 


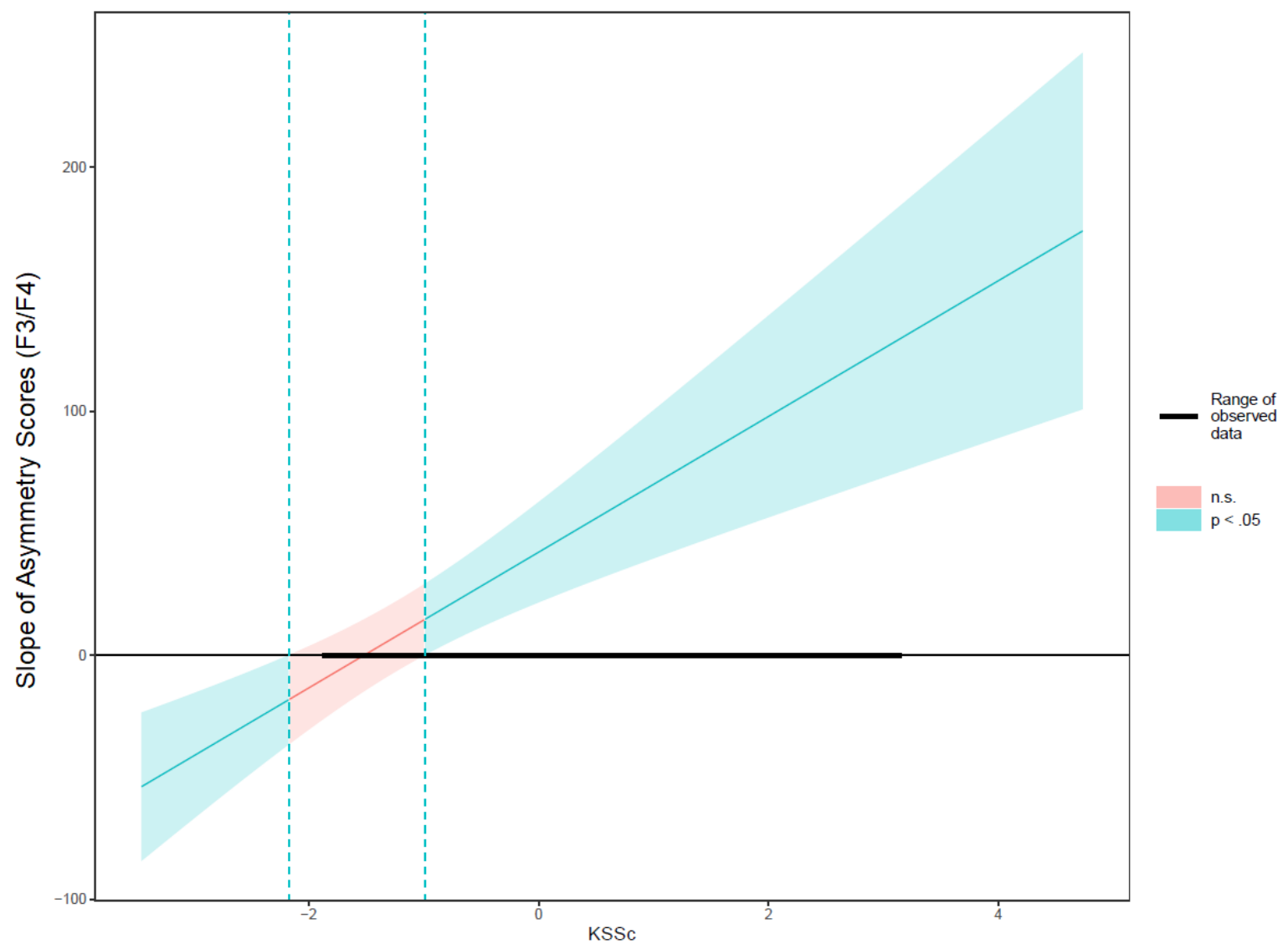

Fig.6. Johnson-Neyman plot for the interaction displayed in Fig.4. The Johnson-Neyman interval encloses all possible slopes for asymmetry scores with $95 \%$ confidence for different values on the KSS. Notice that KSS values are mean-centered (hence, "KSSc"). The Red region indicates the portion of the interval, for which the interaction effect is not significant $(p>0.05)$. In contrast, blue regions indicate statistical significance. Thus, the interaction effect is valid only for KSSc values outside the interval $[-2.17,-0.99]$ (dashed lines). The bold black line superimposed on the zero-line parallel to the $\mathrm{x}$-axis displays the range of $\mathrm{KSSc}$ values considered in the $\operatorname{model}(\min .=-1.86, \max =3.14)$.

correlation to any other rBAS-subscale or to the rBAS itself. This observation had been made by Corr and Cooper (2016) while developing the RST-PQ. The authors argue that BAS behavior entails several sequential subprocesses, i.e., the constructs reflected in each rBASsubscale. While impulsivity manifests during the obtainment of reward, goal-drivepersistence, reward reactivity, and reward interest are relevant processes during the early stages of approach behavior and motivation. The former is akin to emotions of pleasure and joy and the latter represent positive expectations and hope (Corr \& Cooper, 2016). Hence, Impulsivity stands apart theoretically and empirically from other BAS-factors. Nevertheless, a strong correlation between BAS and rBAS was obtained, reflecting the inconsequential changes that the BAS underwent during Gray's and McNaughton's revision of RST (2000). Conversely, the lack of correlation between both BIS constructs could reflect the drastic differences between RST-versions. Finally, as expected, all theoretically unrelated constructs failed to show any significant relationship to each other. In sum, the overall correlational 
pattern is indicative of discriminant and convergent validity among all scales. This notion is further strengthened by the internal consistencies observed here. While differing considerably from one scale to another, questionnaire scores were reliable measurements suitable for interpretation, except for those obtained from rBAS I. Thus, the assessment tools employed here to measure psychological constructs are in line with theoretical deliberations.

\section{Quality Measures}

All quality measures consentaneously suggest superior data quality for EEG-datasets preprocessed with a standardized pipeline compared to performance achieved by manual preprocessing, regardless of eye-condition. Thus, our results met our expectations. This is the more remarkable considering that we chose a rather liberal configuration of preprocessing options in Automagic. To name some examples for comparison, Automagic offers as an alternative to clean_rawdata() the PREP-pipeline (Nima Bigdely-Shamlo et al., 2015) for bad channel detection. Instead of ICLabel the user can opt for the Multiple Artifact Rejection Algorithm (Winkler, Haufe, and Tangermann 2011; Winkler et al., 2014) and/or the robust principal components analysis (rPCA) algorithm developed by Lin et al. (2010) for artifact correction. Pedroni et al. (2019) found that preprocessing workflows akin to ours do not reduce as much noise in resting state EEG-data as workflows that employ these alternatives omitted here. Moreover, notice that the distribution of quality measures was heavily skewed toward zero, especially THV- and CHV-scores. Particularly, no single channel of any dataset preprocessed automatically scored differently than 0 . This means that on average most datasets for most of their run-time did not surpass the $30 \mu \mathrm{V}$ magnitude threshold. It could be argued that other thresholds would have been more sensitive to amplitude variance. Considering that signal peak-to-peak amplitude in adult humans' alpha-rhythm ranges typically between $20 \mu \mathrm{V}$ and $60 \mu \mathrm{V}$ (Orrison, 1995), we argue that setting stricter (i.e., lower) thresholds for data quality assessment is not advisable for alpha rhythms. Automatic preprocessing effectively reduced signal amplitudes to levels below the parameters chosen while manual preprocessing did not. This difference may be explained in part by how both systems approached channel interpolation and the identification of bad channels. Importantly, in the automated pipeline interpolated channels were marked as corrupted at the end of the pipeline. Meanwhile, the manual pipeline employed here did not. Since corrupt channels are reconstructed based on the signal of surrounding sites, the interpolation approach in the standardized pipeline used artifact-reduced channels as a reference, whereas we based our manual interpolation on surrounding raw signals. Additionally, for both eye-conditions, 
automated preprocessing using Automagic identified and interpolated more poor-quality channels than humans via BVA. Automatized preprocessing achieved this in a two-step process: first, by considering three objective parameters (Figure 2), and second, by detecting high-variance channels again after all preprocessing steps but before channel interpolation. In comparison, manual bad channel detection was carried out only once, at the very beginning of the pipeline, and by rejecting only those channels for which the range of the signal's amplitudes were grossly larger than the rest of channels for the vast majority of the recording. Combining all observations, it is then imaginable that Automagic yielded preprocessed datasets with a considerably smaller proportion of noise (e.g., datapoints of high variance) in the signal due to more rigorous and repeated examinations of the data. Furthermore, more corrupt channels identified means more interpolated channels which in turn reduces the ratio of channels of high variance (i.e., smaller $\mathrm{CHV}$-scores). Hence, Automagic reported no nonzero $\mathrm{CHV}$-scores partly because it interpolated so many channels whereas human users failed to identify and interpolate many corrupt channels, increasing the chances of observing at least some high variance channels. Our results show that subtle alterations in preprocessing steps can lead to changes in data quality and highlights the importance of choosing a proper sequence of preprocessing steps.

The fact that manual preprocessing performed poorer than its automated counterpart does not necessarily indicate that BVA-preprocessed datasets should be disregarded. Manual performance was poorer relative to that of an automated software. The magnitude of all quality measures based on amplitude variance are nonetheless negligible. In most of cases, less than $3 \%$ of timepoints presented a high voltage magnitude, and less than $2 \%$ of timepoint variance as well as less than $10 \%$ of channel variance were marked as exceedingly high.

\section{Reliability}

We chose to estimate split-half reliabilities by comparing the first half to the second half of every dataset over other methods (e.g., odd-even halves where recordings consist of segments with eyes closed and segments with eyes open in a different order for every participant) to gain insights on the effect of recording length. Poor reliability could be an indicator of considerable change in alpha power due to tiredness experienced by participants as times goes by (Maltez, Hyllienmark, Nikulin, \& Brismar, 2004). After all, we recorded 10 min. of resting-state EEG, beyond the typical recording length that ranges anywhere from 4 
mins. to 8 mins. in the field of alpha asymmetry research (Smith et al., 2017). On the other hand, we prevented fluctuations in error variance by avoiding the alternation of eye status in a single recording. However, reliability estimates appeared to be more dependent on the preprocessing software than mental fatigue. Without exception, average alpha power stemming from datasets preprocessed by automatized software was outstandingly reliable. In comparison, manually preprocessed average alpha power was not always as reliable and varied considerably from one channel to another. Low reliability at frontal sites during ECrecordings are noteworthy. Of all regions on the scalp, frontal channels are the most contaminated by ocular artifacts (Hagemann, 2004). Even if eyelids are shut and saccades are naturally reduced (in a conscious state), the eyes produce movements capable to inflict amplitude deflections on the EEG-signal. These are nevertheless more difficult to detect visually since they are attenuated compared to movements with opened eyes. Either during artifact rejection or artifact correction, we may have failed to segregate the influence of such subtle artifacts, which in turn compromised the consistency of our measurements at frontal sites. If our speculations are correct, we could deduce that alpha power coming from datasets preprocessed in Automagic did not suffer from reliability issues because standardized preprocessing successfully eliminated these ocular artifacts from the rest of the signal. This could be the case since ICLabel, the algorithm behind the identification and elimination of ICs in Automagic, was trained on several thousands of EEG-recordings and was developed to be sensitive to such artifacts, among many others (Pion-Tonachini et al., 2019). In sum, our hypothesis that single channel average alpha power reliability is invariably high was disputed by our results. Reliability is dependent on channel location, eye-condition, and preprocessing method.

Contrary to single-channel reliability, all estimates for asymmetry scores at frontal electrode pairs were high. This also extends to posterior sites, excepting to scores at P3/P4 coming from EC-recordings, that showed moderate reliability. This is in line with our hypothesis and with past research that found asymmetry scores to be a highly reliable electrophysiological measure (Allen, Urry, Hitt, \& Coan, 2004; Tomarken, Davidson, Wheeler, \& Kinney, 1992; Towers, \& Allen, 2009). In the case of analyses that assume asymmetry scores to be a trait-metric, good reliability is a requirement. Poor reliability would be indicative of asymmetry scores not measuring stable traits, probably influenced by state portions of the total variance.

Past research has criticized reliability measures such as cronbach's alpha or SpearmanBrown corrected split-half reliabilities for being partially dependent on the number and 
duration of 'items', that is, the number of epochs treated as items (Allen et al., 2004; Towers $\&$ Allen, 2009). The longer the epochs, and the more epochs are used, the more accurate the reliability estimate. While each half used here was large (comprising several minutes), we used only two segments. This problem can be overcome if the reliability coefficient is estimated by using the mean of all reliability estimates that are based on all possible split halves. In practice, it is sufficient to take the mean of a representative sample of split-half estimates. Otherwise, the true reliability score is underestimated (Towers \& Allen, 2009). Although the coefficients reported here are underestimations, they were generally so high so that they still indicate that asymmetry scores are outstandingly reliable psychophysiological measures.

In most cases, reliability estimates followed the previously observed pattern that estimates for asymmetry scores are usually lower than reliability estimates from their constituent parts, i.e., alpha power at homologue sites (Allen et al., 2004; Hagemann, 2004; Caruso, 2004). Interestingly, some cases showed the inverted pattern: high estimates for asymmetry scores calculated from sites with low reliability. The reliability estimate for the manually preprocessed EC-pair F3/F4 depicts a drastic example of this. To our knowledge, this is an unprecedented observation. To explain this pattern, the construction of asymmetry scores themselves should be considered. They are difference scores, meaning that although the magnitude of total alpha power varies over time (hence, causing single-channel reliability reduction), this variation is irrelevant for the difference in hemispheric alpha power, as long as power for each hemisphere does not vary independently from its homologue over time as well. Interhemispheric power variance over time seems to be mostly absent in the present sample since the difference in reliability estimates between the majority of homologue sites is marginal. For example, in the aforementioned frontal electrodes exhibiting poor reliability the interhemispheric reliability difference is 0.063 for one pair and 0.067 for the other. Thus, high reliability estimates for asymmetry scores are possible even though their computation is based on not reliable parts. Conversely, the difference in single-channel split-half reliability for electrodes P3 and P4 is large (0.906). Consequently, the asymmetry scores stemming rom these electrodes have the lowest reliability estimate of all pairs.

Consider now the agreement between preprocessing methods and between raters for single electrodes (Table 3 ). Our predictions agree only partly with the results. It becomes evident that only for alpha power at all frontal sites during EC-recordings a moderate to large cross-method/interrater reliability can be observed. For all other cases, alpha power changed depending heavily on the software or rater that extracted it. There are two ways to interpret 
this result. First, they could suggest that, at least for frontal electrodes, comparable levels of alpha power are reproducible if different researchers are working independently on the same set of EEG-data, or even if the software of choice varies between labs. This would come in handy for multi-centered projects, which are almost inevitably characterized by team- and equipment heterogeneity. Especially for those interested in frontal alpha asymmetry research. Caution should be nonetheless taken, as this may be true only under very specific circumstances. For instance, agreement for frontal alpha power coming from EO-datasets would suggest quite the opposite. Furthermore, we extracted power from resting-state EEGrecordings, considering only the canonical alpha band. Our findings might not hold for other frequency ranges or other band partitions, such as the upper and lower alpha band, and/or for experimental designs, where more artifacts are to be dealt with and power is calculated with far fewer datapoints. Neither do our observations extrapolate to other populations, as, for instance, alpha power in children differ from that of adults in several aspects (Srinivasan, 1999). The second interpretation is linked to the reliability issues mentioned above. Poor single channel split-half reliability, as an index of high fluctuation of power variance over time, translates into a reduction of similarity between observations within the same higher order level, i.e., a lower ICC. Lower ICCs would normally suggest greater similarity to other higher order levels. However, in the case of concurrent low split-half reliability, we argue that low ICCs should be interpreted as consistency between observations of the same group at a higher order level so low that is indistinguishable from consistency to other observations at other higher order level groups, rather than as a large consistency to other higher order level groups. Taken together, high interrater reliability, or high agreement between software packages on single channel power cannot be deduced from low ICCs if the corresponding reliability measures are poor. Thus, our hypothesis proposing acceptable agreement can neither be refuted nor confirmed entirely due to part of the results being unsuitable for interpretation. Informative ICCs (i.e., ICCs from channels with at least acceptable underlying split-half reliability), however, did refute our hypothesis adamantly.

Agreement between raters proved to be higher than between preprocessing methods, albeit only by a small margin. Moreover, both reflect high and poor degree of agreement for EC- and EO-conditions, respectively. This observation is, again, valid only for frontal electrodes. Power at all other electrodes showed the inverted pattern. That is, better agreement between software packages than between raters. Notice that agreement was markedly slight in these cases. Thus, our predictions of similar between rater/between software package reliability were contradicted. This contrasts previous research validating 
other automated pipelines that show that both manual and automated approaches to preprocessing yield no sizeable differences in spectral analysis between them (da Cruz et al., 2018; Mariani et al., 2018, Viola et al., 2009). Even in cases where they do, they mostly impact frequency bands at higher and lower ranges than alpha (Duan et al., 2021; Robbins et al., 2020). However, this corpus of research is rather small. The literature focuses much more on the degree of agreement between experts in a clinical context, and how they identify the presence of specific EEG events in recordings, like alpha rhythm frequency, alpha reactivity, anterior-posterior gradients, diffuse slow-wave activity, and even asymmetries, among others (Lodder \& van Putten, 2013). Interrater agreement has been observed to range everything from poor to excellent (Haut et al., 2002, Benbadis et al., 2009, Gerber et al., 2008, Azuma et al., 2003). One of the main reasons for this is a lack of consistency in describing the properties of interest accurately (Azuma et al., 2003). Even automated algorithms identify clinically relevant events like seizures (Rasekhi, Mollaei, Bandarabadi, Teixeira, \& Dourado, 2013), and ERPs related to schizophrenia (Zhang, 2019) with different degrees of accuracy. Even though these are examples of clinical research, we believe that the same visual skills of a clinician are required to identify gross artifacts in basic research. These take part in how EEG-data will turn out for subsequent analysis (Clayson, 2020; Cowley, Korpela, \& Torniainen, 2017). Thus, even though direct comparison between humans and algorithms are scant in the literature, research comparing only humans and research comparing only software tools mentioned above suggest that agreement between humans may be different than agreement between algorithms.

To understand cross-method/interrater reliability concerning asymmetry scores we first must clarify negative values reported for some ICCs (table 3). This might appear surprising given that ICCs, being a ratio of variances, should only range between 0 and 1 . While the true value for any ICC is indeed non-negative, its estimate can be (Taylor, 2010). This is possible because the calculation of ICCs consists of two unbiased estimators, and the ratio of two unbiased estimators is almost always biased (Field ,2005). This bias is negative, leading to negative ICC-estimates when the true value is nearly zero (Taylor, 2010). As Taylor (2010) explained, "negative ICC estimates are possible and can be interpreted as indicating that the true ICC is low, that is, two members chosen randomly from any class vary almost as much as any two randomly chosen members of the whole population." In our case, negative values should then be interpreted as a high agreement between preprocessing methods or between raters. Thus, high agreement on EO-asymmetry scores between manual and standardized preprocessing is observed for all electrode pairs excepting pair P7/P8. 
Conversely, rather low agreement was found for EC-asymmetry scores with an exception for scores at the F3/F4-pair. ICCs throughout all electrode pairs for EC-recordings indicate little to no agreement between raters. The pattern is inverted for EO-recordings, where agreement

is high, except for asymmetry scores obtained from alpha power at P7 and P8. In sum, agreement between software packages was fair whereas agreement between raters was poor for EC-recordings. In the case of EO- recordings, rater/rater and cross-method agreement was similar. Hence, our expectations were refuted by the evidence. Our findings parallel reports in the broader electrophysiological literature that show variability in interrater reliability and variability across EEG-analyses on a variety of EEG-indices. For instance, past research has found variability in source localization and connectivity estimates (Mahjoory et al., 2017), TMS-evoked potentials (Bertazzoli et al., 2021), and even though limited to patients with cerebral ischemia, in brain asymmetry indices (Sheorajpanday, Nagels, Weeren, van Putten, $\&$ De Deyn, 2009). The present work is the first to report discrepancies between preprocessing methods in the resulting alpha asymmetry scores for frontal, parietal, and occipital channels.

\section{Asymmetry Score Distribution}

Since we recruited only participants with no current mental disorders or clinical record thereof, there is no reason to interpret the polarity of zero-deviant distributions under the theoretical framework, that greater relative rightward or leftward activity would indicate the vulnerability to the development of affective disorders such as depression, anxiety, or mania/hypomania (Harmon Jones et al., 2002; Henriques \& Davidson, 1990; Thibodeau, Jorgensen, \& Kim, 2006). In this case, it is more prudent to assume the distribution of asymmetry scores to be a product of random sampling, thus representing asymmetry score distribution at the population level. Asymmetry scores stemming from the electrode pair P7/P8 showed a significant median deviation from zero regardless of eye-condition or preprocessing software, except for the score distribution stemming from the manually preprocessed EO-alpha power. This replicates the work by Ocklenburg and colleagues (2019), who also found strong rightward alpha asymmetry for scores at the P7/P8-pair. Besides, under the same conditions, scores from pairs $\mathrm{F} 3 / \mathrm{F} 4$ and P3/P4 significantly deviated from zero as well. While the score distribution from pairs P7/P8 and P3/P4 was skewed toward positive values, P3/P4's distribution was negatively biased. This is opposed to the observations made by Ocklenburg et al. (2019), as they did not find any other alpha-asymmetric score distribution at the electrode pairs also examined here. This could be due to a number of 
reasons. First, we may have declared asymmetries as significant that the authors did not because we conducted a smaller number of parallel analyses. In their study, they observed several trends to significance at parietal and frontal electrode pairs but did not survive correction to multiple comparisons to more electrode pairs and frequency bands not considered here (Ocklenburg et al., 2019). Furthermore, the authors found that the degree of asymmetry at the alpha band depends on handedness. Hence, differences in LQ-distribution here could also explain discrepancies in asymmetry distribution between both studies. Yet another contributor of result discrepancy could be the preprocessing method applied. While there were no substantial differences between the manual- and standardized approach in the asymmetry score analysis of EC-datasets, a difference in the result pattern for EOdistributions can be observed. However, analyses revealed no main effect for preprocessing software or eye-condition. Our results disputed our predictions. The overall result pattern could be better understood if an eye-condition x software interaction or even an eye-condition x software $\mathrm{x}$ electrode pair interaction were analyzed. Unfortunately, this possibility could not be explored since interaction analysis is not possible for the non-parametric tests implemented here (see 'Limitations').

However, our hypothesis was supported in our second analysis focusing on differences between asymmetry scores stemming from different electrode pairs. Scores at P7/P8 were different from those at F7/F8 and F3/F4. Additionally, scores at P3/P4 deviated from those at F3/F4. All differences are evidently a product of the rightward bias (i.e., on trend more righthemispheric alpha power) and the leftward bias (i.e., on trend more left-hemispheric alpha power) of asymmetry scores at parietal and frontal sites, respectively. Again, this is consistent with previous empirical research and a meta-analysis, both with large sample sizes, that found the same pattern of distribution at the population level in healthy (Ocklenburg et al., 2019) and even clinical samples (van der Vinne, Vollebregt, van Putten, \& Arns, 2017).

\section{Alpha Asymmetry of Personality}

No correlation between asymmetry scores and self-report measures of personality could be found while controlling for gender, handedness, and sleepiness. Alternating software packages or eye-conditions did not alter this result either. Importantly, this observation applies to every electrode pair. For this reason, the hypothesis that this phenomenon is specific to frontal sites can neither be confirmed nor refuted here. Our findings agree with an ever-growing bulk of evidence failing to reproduce this association. In line with our predictions, our failure to find any association between withdrawal tendencies, as reflected in 
the unrevised BIS scale, has been repeatedly reported (Amodio et al., 2008; Berkman \& Lieberman, 2010; Coan \& Allen, 2003a; Coan, Allen, \& Harmon-Jones, 2001; De Pascalis et al., 2013; Harmon-Jones \& Allen, 1997; Hewig et al., 2006; Keune, Bostanov, Kotchoubey, \& Hautzinger, 2012; Kline, Blackhart, Woodward, Williams, \& Schwartz, 2000; Quirin, Gruber, Kuhl, \& Düsing, 2013; Wacker, Chavanon, Leue, \& Stemmler, 2008; Wacker et al., 2010). Furthermore, our results reflect limited evidence that calls into question the widely spread association between BAS and relative greater left cortical activity (Gable et al., 2015; Hewig et al., 2006; Neal \& Gable, 2017; Shackman et al., 2009; Schneider et al., 2016; for reviews see also Coan \& Allen, 2004, and Wacker et al., 2010). The association between resting-EEG activity and FFFS, has been rarely investigated (De Pascalis et al., 2018, 2020; Neal \& Gable, 2017; Threadgill \& Gable, 2018). Only two instances have found any relationship with this scale in an idling mental state (Neal \& Gable, 2017; De Pascalis et al., 2018). Contrary to our expectations based on recent research (De Pascalis et al., 2018, 2020; Gable et al., 2017), both revised versions of BAS and BIS did not correlate with asymmetry scores. Although the literature indicates that trait impulsivity should show a relationship with asymmetry scores (Gable et al., 2015, 2018; Neal \& Gable, 2016, 2019), the present study cannot corroborate such findings. Interestingly, studies supporting this relationship are always experimental setups manipulating situational variables that most probably elicit states of impulsivity. As the capability model predicts (Coan et al., 2006), frontal alpha asymmetry must be elicited strongly enough for associations to other trait variables to be detectable, including impulsivity. The remaining rBAS subscales (RR, RI, and GDP) are underrepresented in the literature, possibly due to their recency. Only the work by De Pascalis and colleagues (2018), on which our hypothesis concerning these scales solely relied on, has succeeded in relating these in any form to asymmetric frontal alpha activity. Nevertheless, our expectations were confronted by null results. Our results highlight the necessity of more replication attempts in future research using plain correlations between resting state EEG and RST-PQ scales to consolidate a verdict whether improvement over research using dated measures of personality is apparent.

We further investigated the possibility of finding an association between personality traits and asymmetric resting state alpha power only under conditions imposed by other variables. This could explain the lack of bivariate relationships. Contrary to our expectations, alpha asymmetry scores at frontal channels F3 and F4 predicted rBIS scores, irrespective of handedness and gender. Sleepiness appeared to moderate this relationship. rBIS scores increased with increasing relative right alpha power, or relative left cortical activity. Increases 
in sleepiness only amplified this relationship. This result is not contemplated by any current theoretical account either. There may be a few reasons for this. Consider the standard error that accompany every estimate in the regression models (Table 5). They are remarkably high. Consequently, the estimation of the confidence bands (Figure 5) was imprecise. Confidence bands indicate the range of values where the true value of an estimate lies to a $95 \%$ degree of confidence. In this case, estimate variability was so high, that even a change in sign could have been possible. It should be noted that this range also changes as the estimate is computed for different values on moderating variables. Changes in sign due to high standard error estimates could be attributed to problems with collinearity. Collinearity is usually unacceptably high when introducing moderation terms into multiple regression models. The easiest and most effective work-around is the mean-centering of variables (Shieh, 2010). Indeed, many interaction terms presented high collinearity, including the ones for the models discussed. Mean-centering of data effectively eliminated concerning levels of multicollinearity. Furthermore, high multicollinearity might not even have consequences for the interpretation of estimates, if the estimate in question stems from an interaction term (Shieh, 2010). We argue that multicollinearity did not bias our calculations and, by extension, our interpretations. Another source of large standard errors of estimates is multivariate nonnormality. If this is the case, then the estimation of regression parameters is not biased, but confidence intervals and $p$-values are (Osborne, 2013). We employed both visual inspection and statistical tests to check for multivariate normality. Importantly, we used the Lilliefors test, a more liberal variant of the Kolmogorov-Smirnoff test (Ghasemi \& Zahediasl, 2012). The test has been heavily criticized in the past for being too conservative, i.e., being unnecessarily sensitive to outliers even if the normality assumption is not violated. Some authors argue that the test is not any more informative than subjective visual inspection if done right, for instance via Q-Q-plots (Ghasemi \& Zahediasl, 2012). Indeed, Q-Q plots did not raise any concerns here. Furthermore, other authors argue that when the sample size is large enough (usually $n>30$ ), deviations from normality can be neglected (Altman \& Bland, 1995). Issues with normality in our data can be then disregarded. In contrast, heteroskedasticity analysis delivered mixed results. While the assumption of homoskedasticity could not be rejected through the Breusch-Pagan test ( $p$ 's $>0.1$ ), plots displaying (standardized) residuals against fitted values revealed uneven variance distribution. Residual variance being highest around the mean of fitted values and lowest towards the end of the range of fitted values. Like normality violations, heteroskedasticity bias the estimation of confidence intervals and p-values. This pattern could indicate model misspecification. One 
alternative would be to examine possible non-linear relationships between asymmetry scores and personality constructs. To our knowledge, this poses unexplored territory in this line of research. For the same reasons, we did not consider it during model building. Non-linear relationships open nevertheless an interesting avenue for future research. We decided not to deem our models as unreliable when at least one approach to assumption testing contradicted another one indicating issues with any linear regression assumption, as was the case for homo-/heteroskedasticity. In sum, the models presented here should be interpreted with caution, even though they probably fulfill all model assumptions tested. Assuming faithful model fitting, however, our results represent an unprecedented event where the (revised) BIS not only relates to common asymmetric frontal alpha power but also shows an inverted pattern. Namely, greater relative left frontal activity.

Our predictions on the influence of covariates were partially disconfirmed. As discussed above, only sleepiness hinted at a moderation. Gender did not show a significant moderating role. Gender has been considered in several studies to be a determinant of alpha asymmetry in relation to psychological constructs (Wacker et al., 2010; De Pascalis et al., 2018, 2020). These studies, however, discussed attraction between participants and experimenters to be the mechanism of action to influence concurrent EEG alpha power, not plain sex differences in EEG spectral characteristics. Although a tentative possibility, the authors assumed heterosexual attraction during data analysis and -interpretation, neglecting variability in sexual orientation and subjective feeling of attraction at the time of testing. While we did not document any of these aspects either, we still included gender (as indicative of biological sex) in our models since alpha asymmetry fluctuates during the menstrual cycle (Hwang et al., 2008, Huang et al., 2015). As we did not experimentally manipulate the status of menstrual cycle either, hormonal-related changes in asymmetric power went uncontrolled. Heterogeneity in menstrual phase status and even in experimenter/participant attraction might have possibly masked the associations of interest. After all, roughly a half of the variance is explained by situational factors (Hagemann et al., 2002; Hagemann, Hewig, Seifert, Naumann, \& Bartussek, 2005).

Moreover, handedness also failed to show any involvement in alpha asymmetry of personality. Although handedness has important functional consequences across many asymmetric phenomena in humans (Ocklenburg \& Güntürkun, 2017; Ockelnburg et al., 2019), it has been overlooked almost entirely by alpha asymmetry experts. Most of them disregard any possible impact of handedness on the results as negligible (Vecchio \& De Pascalis., 2020). This notion is supported here. There have been, however, few instances 
where handedness has been considered, and indeed a modulating role has been identified (Papousek \& Schulter, 1999; Propper, Pierce, Geisler, Christman, \& Bellorado, 2012). However, these studies limited themselves to the affect regulation capabilities of righthanders. We expand the debate by including left-handers in our sample and in the context of personality research. If alpha asymmetry relations to psychological traits were an inherent feature of right-handers, we could still have found significant interaction effects, but limited to positive LQ-scores. This was not the case. In sum, we support the general notion that handedness exerts a futile influence on the alpha asymmetry of personality. It should be noted that our sample consisted of too few left-handers (12). We encourage future work in this area to actively increase their left-handed subpopulation to confidently draw conclusions on the effect of handedness.

Of course, all these deliberations can be supported by our data only when excluding influential values out of the models. Since we already excluded implausible outliers before data analysis, further data reduction occurred based solely on statistical grounds, not theoretical ones. For this reason, we favor the conclusion that no link between resting state alpha asymmetry and personality traits could be reproduced in the present work. We chose to carry out sensitivity analyses only to conserve the general linear model as our core statistical basis. Alternative approaches to multiple regression that appropriately deal with influential values/outliers without excluding them from analysis include mixture distribution analyses, robust regression analysis, PCA or partial least squares regression (Eid et al., 2015).

\section{Limitations}

Comparability between manual- and automated preprocessing was hampered due to gross differences in methodology. Standardized preprocessing using Automagic approached data-cleaning by means of artifact correction whereas for manual preprocessing in BVA we opted for both artifact correction and rejection. Under the assumption that any datapoint is a linear combination of signal and noise (Islam et al., 2016), the portion of signal within an artifact-contaminated segment is lost when practicing artifact rejection. Hence, manually preprocessed datasets potentially took a larger signal-loss simply by applying one dataexcluding step that its automatized homologue did not. It could be argued that it does not necessarily raise concerns for the preprocessing quality if the manual data-distorting step (i.e., ICA) differed from the automated one such that the finished preprocessed dataset is not distinct from that obtained using automatic preprocessing. We believe that this is not the case since the ICA-algorithm integrated in both programs is based on the same mathematical 
grounds, making the signal-unmixing into ICs sufficiently similar. One trivial solution would have been to abolish artifact rejection in BVA. However, this is not recommendable since gross artifacts greatly reduce the ICA's capability to separate input-data into single ICs (Dimigen, 2020), which in turn would make manual IC-rejection increasingly difficult. On the other hand, data decomposition achieved by ICA is most effective the longer the EEG-data training set is (Plank, 2014). In Automagic this dilemma (reducing data but keeping as much as possible to optimize the ICA's performance) is overcome by ICLabel for it can accurately classify ICs with a high degree of tolerance to high-variance components (Pion-Tonachini, et al. 2019) without shortening the dataset's length. Thus, automated extraction of IC's was more effective than the manual implementation since the size of training sets fed to BVA were shorter than Automagic's.

Furthermore, the sequence of single preprocessing steps varied between preprocessing programs. Channel interpolation occurred immediately after channel rejection on raw EEGdata at the beginning of the manually set-up pipeline whereas the automated approach implemented channel interpolation as the very last step down the pipeline. This potentially contributed to the stark differences in the quality of preprocessed EEG-data, which in turn could impact data analysis as well. The latter point remains nonetheless speculative since our analyses do not allow such inferences.

We neglected the common practice of counterbalancing the order of eye-conditions. The potential effect of first committing to a 10 mins. resting state with eyes open followed by 10 mins. with eyes closed could have been easily canceled out by randomly assigning one of two possible instruction orders to each participant (EO-then-EC or EC-then-EO).

Our results might be heavily influenced by the expertise of both human users in charge of all EEG-data handling. While one experimenter was first introduced to practical EEGpreprocessing during this project, the other experimenter had already accumulated elementary knowledge and practical skills in artifact rejection due to duties as a research assistant and prior to data handling in the present work. Both experimenters are nevertheless regarded as novice users of EEG-handling software. For example, human users only received elementary training on IC-identification limited to eye-blinks, saccades, and cardiac activity. Any other artifactual source was merely identified based on subjective impressions that ICs are potentially of non-neural nature (comparable to an "other"-category) without any further differential knowledge of IC characteristics. Artifact correction via ICLabel, however, further distinguishes muscle activity, line noise, as well as channel noise from other artifactual ICs. 
Differences between manual and standardized preprocessing could have turned out smaller had data been handled by users with greater expertise, just as observed elsewhere (Hatz et al., 2015).

Our choice of statistical analyses was heavily influenced by the non-normal distribution of questionnaire scores, quality measures, and asymmetry scores obtained here. Non-parametric tests are widely accepted as robust alternatives to parametric ones for when normality in the variables of interest is not given (Harwell, 1988). However, this comes at the price of giving up some analytical advantages of parametric tests. For example, repeated measures ANOVAs can accommodate multifactorial study designs and between-factor interactional analyses. Their non-parametric equivalent, the Friedman test, allow only onefactorial analyses. Consequently, no interactions can be examined. Although it has been argued that violations of the normality assumptions for analyses using the general linear model may not distort analysis validity drastically, we opted for more reliable, though less sophisticated, statistical values. Thus, we may have missed potential insights on the behavior of asymmetry score distribution that interaction analyses would have allowed.

As mentioned earlier, we underestimated split-half reliability coefficients of alpha power and asymmetry scores by using only two halves for calculations. Analyses by Towers and Allen (2009) found that each segment should be iteratively regrouped in new halves and for each half a Spearman-Brown corrected estimate should be computed. The mean of 1000 iterations represents the true score appropriately. Future research should partition EEGrecordings into more segments. The number and length of segments should be chosen based on the total length of the dataset and the choice of reference (Towers \& Allen, 2009).

The problem of correction after multiple testing decisively determined our reached conclusions. The Bonferroni correction, while simple, is infamously strict. Great debates have been held for when, if at all, the Bonferroni method should be applied (Cabin \& Mitchell, 2000; Nakagawa, 2004; Perneger, 1998). Still, no consensus has been reached (VanderWeele $\&$ Mathur, 2019). In times of a replication crisis in many fields of research, where too many false positive results are being reported, we wanted to reject the null hypothesis only under the strictest conditions. The Bonferroni method indeed guarantees, within a hypothesis-testing framework, to eliminate type I errors entirely (VanderWeele \& Mathur, 2019). This comes of course with the great disadvantage of inflating type II errors, also topical in the current state of affairs in academia. By being too conservative, statistical power rapidly diminishes with increasing number of comparisons. Considering customs in the field of psychophysiology, the 
number of comparisons (up to 180) in our analyses is high. Null findings may also be attributed to the small sample size, which also diminishes statistical power. For example, to accomplish a satisfactory, though arbitrarily chosen, power level of 0.8 in our two-tailed partial correlation analyses, assuming an intermediate effect size $|\rho|$ of 0.3 and setting $\alpha$ to 0.5 , we would have required a total of 83 participants. With only 47 participants, our analyses would have been able to detect moderate effects with a power level of 0.55 . In the case of $t$ tests for single predictors in multiple regression models, estimations are more optimistic. To achieve $1-\beta=0.8$, a sample size of 55 participants would have been necessary. In reality, our models were powered to a level of up to 0.73 in order to detect effect sizes $f^{2}$ as small as 0.15 . However, these calculations do not take Bonferroni corrections into account. In sum, a combination of a modest sample size and a strict null hypothesis rejection criterium may have contributed to considerable power losses, which in turn possibly occluded significant findings.

\section{Implications for the \#EEGmanylabs Resting State EEG Asymmetry Project}

Automatic preprocessing probably reduced more noise and excluded less target-signal than its manual counterpart partly due to the differences in artifact rejection/correction discussed above. Hence, data made available by automated pipelines is preferable. However, differently preprocessed EEG-data yielded subtle differences in other aspects like reliability. These were nonetheless case specific. They depended on the eye-condition and the electrode/electrode pair in question. Differences in asymmetry score distribution were not substantial and proved to be a reliable biomarker suitable for alpha asymmetry research. Consequently, even when automated algorithms perform differently than subjectively guided approaches during EEG-preprocessing, results are not altered in profound ways. We see therefore no reason not to implement standardized pipelines in long-term multicentered projects such as \#EEGManyLabs Resting State EEG Asymmetry. We wish to note that our opinion is best applicable to the analysis of resting state frontal EEG asymmetry and does not extrapolate to the range of topics to be handled by \#EEGManyLabs.

Our study reiterates the importance of sufficiently powered analyses for the upcoming replication attempts by the collaborating labs of the \#EEGManyLabs Resting State EEG Asymmetry project. Given that sample sizes above 40 may be considered large by many collaborating sites due to their strain on research resources (time, funding, equipment, researcher availability, etc.), we urge replication teams to distribute data collection duties with 
other "lab buddies" that share the same equipment (e.g., identical amplifier and electrode models; Pavlov et al., 2021). The attainment of high-powered analyses should be prioritized.

While there are good indications that asymmetric alpha power is a stable trait that measures individual differences, alpha asymmetry is probably susceptible to situational factors. We recommend taking as many relevant state-dependent variables into account as possible if studies set out to examine relationships at the trait level.

\section{Conclusions}

Our analyses corroborate current speculations that different preprocessing methods can lead to different outcomes. In this study, we found substantial differences in data quality after implementing a fully automated approach to resting state EEG-data preprocessing and another subjected to human intuition and judgement. In all regards, the automated approach reduced variance in EEG-data to a more satisfactory degree. Future research could benefit from the greatest feat of automatization: unbiased, reproducible, time-efficient, resource-sparing datamanagement.

Difficulties to replicate findings out of highly popular subjects in psychophysiology were made apparent here. Our results suggest that the relationship between alpha asymmetries and psychological constructs is indeed not robust. Even with self-report measures based on a sound theoretical basis we could hardly detect any effect. Accordingly, our observations are most likely to favor the capability model (Coan et al., 2006) of EEG alpha asymmetry over the approach/withdrawal models (Davidson, 1993, 1998; Harmon-Jones, 2003), the bilateral BAS model (Hewig, 2004, 2005, 2006), and the BBMAA (Wacker et al., 2003, 2008, 2010). 


\section{References}

Ahern, G. L., \& Schwartz, G. E. (1985). Differential lateralization for positive and negative emotion in the human brain: EEG spectral analysis. Neuropsychologia, 23(6), 745-755.

Åkerstedt, T., \& Gillberg, M. (1990). Subjective and objective sleepiness in the active individual. International journal of neuroscience, 52(1-2), 29-37.

Alin, A. (2010). Multicollinearity. Wiley Interdisciplinary Reviews: Computational Statistics, 2(3), 370-374.

Allen, J. J., Coan, J. A., \& Nazarian, M. (2004). Issues and assumptions on the road from raw signals to metrics of frontal EEG asymmetry in emotion. Biological psychology, 67(1-2), 183-218.

Allen, J. J., Keune, P. M., Schönenberg, M., \& Nusslock, R. (2018). Frontal EEG alpha asymmetry and emotion: From neural underpinnings and methodological considerations to psychopathology and social cognition. Psychophysiology, 55(1).

Allen, J. J., Urry, H. L., Hitt, S. K., \& Coan, J. A. (2004). The stability of resting frontal electroencephalographic asymmetry in depression. Psychophysiology, 41(2), 269-280.

Ally, B. A., \& Budson, A. E. (2007). The worth of pictures: Using high density event-related potentials to understand the memorial power of pictures and the dynamics of recognition memory. NeuroImage, 35(1), 378-395.

Altman, D. G., \& Bland, J. M. (1995). Statistics notes: the normal distribution. Bmj, 310(6975), 298.

Amodio, D. M., Master, S. L., Yee, C. M., \& Taylor, S. E. (2008). Neurocognitive components of the behavioral inhibition and activation systems: Implications for theories of self-regulation. Psychophysiology, 45(1), 11-19.

Azuma, H., Hori, S., Nakanishi, M., Fujimoto, S., Ichikawa, N., \& Furukawa, T. A. (2003). An intervention to improve the interrater reliability of clinical EEG interpretations. Psychiatry and clinical neurosciences, 57(5), 485-489.

Babiloni, C., Marzano, N., Lizio, R., Valenzano, A., Triggiani, A. I., Petito, A., ... \& Del Percio, C. (2011). Resting state cortical electroencephalographic rhythms in subjects with normal and abnormal body weight. Neuroimage, 58(2), 698-707. 
Baker, M. (2016). Reproducibility crisis. Nature, 533(26), 353-66.

Balconi, M. (2011). Frontal brain oscillation modulation in facial emotion comprehension: The role of reward and inhibitory systems in subliminal and supraliminal processing. Journal of Cognitive Psychology, 23(6), 723-735.

Balconi, M., \& Mazza, G. (2009). Brain oscillations and BIS/BAS (behavioral inhibition/activation system) effects on processing masked emotional cues: ERS/ERD and coherence measures of alpha band. International Journal of Psychophysiology, 74(2), 158165.

Baldwin, S. A. (2017). Improving the rigor of psychophysiology research. International Journal of Psychophysiology, 111, 5-16.

Begley, C. G., \& Ellis, L. M. (2012). Raise standards for preclinical cancer research. Nature, 483(7391), 531-533.

Bell, A. J., \& Sejnowski, T. J. (1995). An information-maximization approach to blind separation and blind deconvolution. Neural computation, 7(6), 1129-1159.

Bem, D. J. (2011). Feeling the future: experimental evidence for anomalous retroactive influences on cognition and affect. Journal of personality and social psychology, 100(3), 407.

Benbadis, S. R., LaFrance, W. C., Papandonatos, G. D., Korabathina, K., Lin, K., \& Kraemer, H. C. (2009). Interrater reliability of EEG-video monitoring. Neurology, 73(11), 843-846.

Berkman, E. T., \& Lieberman, M. D. (2010). Approaching the bad and avoiding the good: Lateral prefrontal cortical asymmetry distinguishes between action and valence. Journal of cognitive neuroscience, 22(9), 1970-1979.

Bertazzoli, G., Esposito, R., Mutanen, T. P., Ferrari, C., Ilmoniemi, R. J., Miniussi, C., \& Bortoletto, M. (2021). The impact of artifact removal approaches on TMS-EEG signal. NeuroImage, 118272.

Bigdely-Shamlo, N., Mullen, T., Kothe, C., Su, K. M., \& Robbins, K. A. (2015). The PREP pipeline: standardized preprocessing for large-scale EEG analysis. Frontiers in neuroinformatics, 9, 16.

Blanz, M. (2015). Forschungsmethoden und Statistik für die Soziale Arbeit: Grundlagen und Anwendungen. Kohlhammer. 
Botvinik-Nezer, R., Holzmeister, F., Camerer, C. F., Dreber, A., Huber, J., Johannesson, M., ... \& Rieck, J. R. (2020). Variability in the analysis of a single neuroimaging dataset by many teams. Nature, 582(7810), 84-88.

Buss, A. H., \& Perry, M. (1992). The aggression questionnaire. Journal of personality and social psychology, 63(3), 452.

Button, K. S., Ioannidis, J. P., Mokrysz, C., Nosek, B. A., Flint, J., Robinson, E. S., \& Munafò, M. R. (2013). Power failure: why small sample size undermines the reliability of neuroscience. Nature reviews neuroscience, 14(5), 365-376.

Cabin, R. J., \& Mitchell, R. J. (2000). To Bonferroni or not to Bonferroni: when and how are the questions. Bulletin of the ecological society of America, 81(3), 246-248.

Cannon, R. L., Baldwin, D. R., Shaw, T. L., Diloreto, D. J., Phillips, S. M., Scruggs, A. M., \& Riehl, T. C. (2012). Reliability of quantitative EEG (qEEG) measures and LORETA current source density at 30 days. Neuroscience letters, 518(1), 27-31.

Carp, J. (2012). On the plurality of (methodological) worlds: estimating the analytic flexibility of FMRI experiments. Frontiers in neuroscience, 6, 149.

Caruso, J. C. (2004). A comparison of the reliabilities of four types of difference scores for five cognitive assessment batteries. European Journal of Psychological Assessment, 20(3), 166-171.

Carver, C. S. (2004). Negative affects deriving from the behavioral approach system. Emotion, 4(1), 3.

Carver, C. S., \& Scheier, M. F. (2012). Attention and self-regulation: A control-theory approach to human behavior. Springer Science \& Business Media.

Carver, C. S., \& White, T. L. (1994). Behavioral inhibition, behavioral activation, and affective responses to impending reward and punishment: the BIS/BAS scales. Journal of personality and social psychology, 67(2), 319.

Center for Open Science (n.d.) The TOP Guidelines were created by journals, funders, and societies to align scientific ideals with practices. Retrieved from https://www.cos.io/initiatives/top-guidelines 
Clayson, P. E. (2020). Moderators of the internal consistency of error-related negativity scores: A meta-analysis of internal consistency estimates. Psychophysiology, 57(8), e13583.

Clayson, P. E., Carbine, K. A., Baldwin, S. A., \& Larson, M. J. (2019). Methodological reporting behavior, sample sizes, and statistical power in studies of event-related potentials: Barriers to reproducibility and replicability. Psychophysiology, 56(11), e13437.

Coan, J. A., \& Allen, J. J. (2003a). Frontal EEG asymmetry and the behavioral activation and inhibition systems. Psychophysiology, 40(1), 106-114.

Coan, J. A., \& Allen, J. J. (2004). Frontal EEG asymmetry as a moderator and mediator of emotion. Biological psychology, 67(1-2), 7-50.

Coan, J. A., \& Allen, J. J. B. (2003b). The state and trait nature of frontal EEG asymmetry in emotion. In K. Hugdahl \& R. J. Davidson (Eds.), The asymmetrical brain (pp. 565-615). MIT Press.

Coan, J. A., Allen, J. J., \& Harmon-Jones, E. (2001). Voluntary facial expression and hemispheric asymmetry over the frontal cortex. Psychophysiology, 38(6), 912-925.

Coan, J. A., Allen, J. J., \& McKnight, P. E. (2006). A capability model of individual differences in frontal EEG asymmetry. Biological psychology, 72(2), 198-207.

Cohen, M. X. (2017). Rigor and replication in time-frequency analyses of cognitive electrophysiology data. International Journal of Psychophysiology, 111, 80-87.

Conrad, C., \& Bailey, L. (2020). What Can NeuroIS Learn from the Replication Crisis in Psychological Science?. In Information Systems and Neuroscience (pp. 129-135). Springer, Cham.

Corr, P. J., \& Cooper, A. J. (2016). The reinforcement sensitivity theory of personality questionnaire (RST-PQ): development and validation. Psychological assessment, 28(11), 1427.

Corr, P. J., \& McNaughton, N. (2008). Reinforcement Sensitivity Theory and personality. In P. J. Corr (Ed.), The reinforcement sensitivity theory of personality (pp. 155-187). Cambridge University Press.

Corr, P.J. (n.d.). Retrieved from http://www.philipcorr.net/rst-pg77.html 
Corsi-Cabrera, M., Galindo-Vilchis, L., Del-Río-Portilla, Y., Arce, C., \& Ramos-Loyo, J. (2007). Within-subject reliability and inter-session stability of EEG power and coherent activity in women evaluated monthly over nine months. Clinical Neurophysiology, 118(1), 9-21.

Cowley, B. U., Korpela, J., \& Torniainen, J. (2017). Computational testing for automated preprocessing: a Matlab toolbox to enable large scale electroencephalography data processing. PeerJ Computer Science, 3, e108.

Curran, T. (2000). Brain potentials of recollection and familiarity. Memory \& cognition, 28(6), 923-938.

da Cruz, J. R., Chicherov, V., Herzog, M. H., \& Figueiredo, P. (2018). An automatic preprocessing pipeline for EEG analysis (APP) based on robust statistics. Clinical Neurophysiology, 129(7), 1427-1437.

d'Alfonso, A. A., van Honk, J., Hermans, E., Postma, A., \& de Haan, E. H. (2000). Laterality effects in selective attention to threat after repetitive transcranial magnetic stimulation at the prefrontal cortex in female subjects. Neuroscience letters, 280(3), 195-198.

Davidson, R. J. (1998). Affective style and affective disorders: Perspectives from affective neuroscience. Cognition \& Emotion, 12(3), 307-330.

Davidson, R. J., Ekman, P., Saron, C. D., Senulis, J. A., \& Friesen, W. V. (1990). Approachwithdrawal and cerebral asymmetry: emotional expression and brain physiology: I. Journal of personality and social psychology, 58(2), 330.

Davidson, R. J., Schwartz, G. E., Saron, C., Bennett, J., \& Goleman, D. J. (1979). Frontal versus parietal EEG asymmetry during positive and negative affect. Psychophysiology. 16(2), 202-203.

Davidson, R.J. (1992). Emotion and affective style: Hemispheric substrates. Psychological Science, 3, 39-43.

Davidson, R.J. (1993). Cerebral asymmetry and emotion: Conceptual and methodological conundrums. Cognition and Emotion, 7, 115-138.

Dawes, R. M., Faust, D., \& Meehl, P. E. (1989). Clinical versus actuarial judgment. Science, 243(4899), 1668-1674. 
De Pascalis, V., Cozzuto, G., Caprara, G. V., \& Alessandri, G. (2013). Relations among EEGalpha asymmetry, BIS/BAS, and dispositional optimism. Biological psychology, 94(1), 198-209.

De Pascalis, V., Cozzuto, G., Caprara, G. V., \& Alessandri, G. (2013). Relations among EEGalpha asymmetry, BIS/BAS, and dispositional optimism. Biological psychology, 94(1), 198-209.

De Pascalis, V., Sommer, K., \& Scacchia, P. (2018). Resting frontal asymmetry and reward sensitivity theory motivational traits. Scientific reports, 8(1), 1-9.

DeLong, K. A., Urbach, T. P., \& Kutas, M. (2017). Is there a replication crisis? Perhaps. Is this an example? No: a commentary on Ito, Martin, and Nieuwland (2016). Language, Cognition and Neuroscience, 32(8), 966-973.

Dilger, S., Straube, T., Mentzel, H. J., Fitzek, C., Reichenbach, J. R., Hecht, H., ... \& Miltner, W. H. (2003). Brain activation to phobia-related pictures in spider phobic humans: an event-related functional magnetic resonance imaging study. Neuroscience letters, 348(1), 29-32.

Dimigen, O. (2020). Optimizing the ICA-based removal of ocular EEG artifacts from free viewing experiments. NeuroImage, 207, 116117.

Eid, M., Gollwitzer, M., \& Schmitt, M. (2015). Statistik und forschungsmethoden. Beltz.

Field, A. P. (2005), “Intraclass Correlation”, Encyclopedia of Statistics in Behavioral Science (pp. 948-954). John Wiley \& Sons.

Fields, E. C., \& Kuperberg, G. R. (2020). Having your cake and eating it too: Flexibility and power with mass univariate statistics for ERP data. Psychophysiology, 57(2), e13468.

Flexer, A., Bauer, H., Pripfl, J., \& Dorffner, G. (2005). Using ICA for removal of ocular artifacts in EEG recorded from blind subjects. Neural Networks, 18(7), 998-1005.

Francis, G. (2012a). Too good to be true: Publication bias in two prominent studies from experimental psychology. Psychonomic bulletin \& review, 19(2), 151-156.

Francis, G. (2012b). Publication bias and the failure of replication in experimental psychology. Psychonomic Bulletin \& Review, 19(6), 975-991. 
Frank, M. C., Bergelson, E., Bergmann, C., Cristia, A., Floccia, C., Gervain, J., ... \& Yurovsky, D. (2017). A collaborative approach to infant research: Promoting reproducibility, best practices, and theory-building. Infancy, 22(4), 421-435.

Gable, P. A., Mechin, N. C., Hicks, J. A., \& Adams, D. L. (2015). Supervisory control system and frontal asymmetry: neurophysiological traits of emotion-based impulsivity. Social cognitive and affective neuroscience, 10(10), 1310-1315.

Gable, P. A., Neal, L. B., \& Threadgill, A. H. (2018). Regulatory behavior and frontal activity: Considering the role of revised-BIS in relative right frontal asymmetry. Psychophysiology, 55(1), e12910.

Gerber, P. A., Chapman, K. E., Chung, S. S., Drees, C., Maganti, R. K., Ng, Y. T., ... \& Kerrigan, J. F. (2008). Interobserver agreement in the interpretation of EEG patterns in critically ill adults. Journal of Clinical Neurophysiology, 25(5), 241-249.

Ghasemi, A., \& Zahediasl, S. (2012). Normality tests for statistical analysis: a guide for nonstatisticians. International journal of endocrinology and metabolism, 10(2), 486.

Gianotti, L. R., Knoch, D., Faber, P. L., Lehmann, D., Pascual-Marqui, R. D., Diezi, C., ... \& Fehr, E. (2009). Tonic activity level in the right prefrontal cortex predicts individuals' risk taking. Psychological science, 20(1), 33-38.

Gilbert, D. T., King, G., Pettigrew, S., \& Wilson, T. D. (2016). Comment on "Estimating the reproducibility of psychological science”. Science, 351(6277), 1037-1037.

Gray, J. A. (1982). The Neuropsychology of Anxiety: An Enquiry Into the Functions of the Septo-hippocampal System. Oxford University Press.

Gray, J.A. and McNaughton, N. (2000). The neuropsychology of anxiety: an enquiry into the functions of the septo-hippocampal system. Oxford University Press.

Hagemann, D. (2004). Individual differences in anterior EEG asymmetry: methodological problems and solutions. Biological psychology, 67(1-2), 157-182.

Hagemann, D., Hewig, J., Seifert, J., Naumann, E., \& Bartussek, D. (2005). The latent statetrait structure of resting EEG asymmetry: Replication and extension. Psychophysiology, 42(6), 740-752. 
Hagemann, D., Hewig, J., Seifert, J., Naumann, E., \& Bartussek, D. (2005). The latent statetrait structure of resting EEG asymmetry: Replication and extension. Psychophysiology, 42(6), 740-752.

Hagemann, D., Naumann, E., Becker, G., Maier, S., \& Bartussek, D. (1998). Frontal brain asymmetry and affective style: A conceptual replication. Psychophysiology, 35(4), 372388.

Hagemann, D., Naumann, E., Thayer, J. F., \& Bartussek, D. (2002). Does resting electroencephalograph asymmetry reflect a trait? An application of latent state-trait theory. Journal of personality and social psychology, 82(4), 619.

Harmon-Jones, E. (2003). Clarifying the emotive functions of asymmetrical frontal cortical activity. Psychophysiology, 40(6), 838-848.

Harmon-Jones, E. (2004). On the relationship of frontal brain activity and anger: Examining the role of attitude toward anger. Cognition and Emotion, 18(3), 337-361.

Harmon-Jones, E., \& Allen, J. J. (1997). Behavioral activation sensitivity and resting frontal EEG asymmetry: covariation of putative indicators related to risk for mood disorders. Journal of abnormal psychology, 106(1), 159.

Harmon-Jones, E., \& Allen, J. J. (1998). Anger and frontal brain activity: EEG asymmetry consistent with approach motivation despite negative affective valence. Journal of personality and social psychology, 74(5), 1310.

Harmon-Jones, E., \& Gable, P. A. (2018). On the role of asymmetric frontal cortical activity in approach and withdrawal motivation: An updated review of the evidence. Psychophysiology, 55(1), e12879.

Harmon-Jones, E., \& Sigelman, J. (2001). State anger and prefrontal brain activity: evidence that insult-related relative left-prefrontal activation is associated with experienced anger and aggression. Journal of personality and social psychology, 80(5), 797.

Harmon-Jones, E., Abramson, L. Y., Sigelman, J., Bohlig, A., Hogan, M. E., \& HarmonJones, C. (2002). Proneness to hypomania/mania symptoms or depression symptoms and asymmetrical frontal cortical responses to an anger-evoking event. Journal of personality and social psychology, 82(4), 610. 
Harmon-Jones, E., Gable, P. A., \& Peterson, C. K. (2010). The role of asymmetric frontal cortical activity in emotion-related phenomena: A review and update. Biological psychology, 84(3), 451-462.

Harmon-Jones, E., Gable, P. A., \& Peterson, C. K. (2010). The role of asymmetric frontal cortical activity in emotion-related phenomena: A review and update. Biological psychology, 84(3), 451-462.

Harmon-Jones, E., Harmon-Jones, C., Fearn, M., Sigelman, J. D., \& Johnson, P. (2008). Left frontal cortical activation and spreading of alternatives: tests of the action-based model of dissonance. Journal of personality and social psychology, 94(1), 1.

Harwell, M. R. (1988). Choosing between parametric and nonparametric tests. Journal of Counseling \& Development, 67(1), 35-38.

Hatz, F., Hardmeier, M., Bousleiman, H., Rüegg, S., Schindler, C., \& Fuhr, P. (2015). Reliability of fully automated versus visually controlled pre-and post-processing of resting-state EEG. Clinical Neurophysiology, 126(2), 268-274.

Haut, S. R., Berg, A. T., Shinnar, S., Cohen, H. W., Bazil, C. W., Sperling, M. R., ... \& Spencer, S. S. (2002). Interrater reliability among epilepsy centers: multicenter study of epilepsy surgery. Epilepsia, 43(11), 1396-1401.

Henriques, J. B., \& Davidson, R. J. (1990). Regional brain electrical asymmetries discriminate between previously depressed and healthy control subjects. Journal of abnormal psychology, 99(1), 22.

Herron, J. E., Quayle, A. H., \& Rugg, M. D. (2003). Probability effects on event-related potential correlates of recognition memory. Cognitive Brain Research, 16(1), 66-73.

Hewig, J., Hagemann, D., Seifert, J., Naumann, E., \& Bartussek, D. (2004). On the selective relation of frontal cortical asymmetry and anger-out versus anger-control. Journal of personality and social psychology, 87(6), 926.

Hewig, J., Hagemann, D., Seifert, J., Naumann, E., \& Bartussek, D. (2005). The relationship of cortical activity and personality in a reinforced go-nogo paradigm. Journal of Individual Differences, 26(2), 86-99.

Hewig, J., Hagemann, D., Seifert, J., Naumann, E., \& Bartussek, D. (2006). The relation of cortical activity and BIS/BAS on the trait level. Biological psychology, 71(1), 42-53. 
Hortensius, R., Schutter, D. J., \& Harmon-Jones, E. (2012). When anger leads to aggression: induction of relative left frontal cortical activity with transcranial direct current stimulation increases the anger-aggression relationship. Social cognitive and affective neuroscience, 7(3), 342-347.

Huang, Y., Zhou, R., Cui, H., Wu, M., Wang, Q., Zhao, Y., \& Liu, Y. (2015). Variations in resting frontal alpha asymmetry between high-and low-neuroticism females across the menstrual cycle. Psychophysiology, 52(2), 182-191.

Hwang, R. J., Chen, L. F., Yeh, T. C., Tu, P. C., Tu, C. H., \& Hsieh, J. C. (2008). The resting frontal alpha asymmetry across the menstrual cycle: a magnetoencephalographic study. Hormones and behavior, 54(1), 28-33.

Ioannidis, J. P. (2005). Why most published research findings are false. PLoS medicine, 2(8), e124.

Islam, M. K., Rastegarnia, A., \& Yang, Z. (2016). Methods for artifact detection and removal from scalp EEG: A review. Neurophysiologie Clinique/Clinical Neurophysiology, 46(4-5), 287-305.

Ito, A., Martin, A. E., \& Nieuwland, M. S. (2017). How robust are prediction effects in language comprehension? Failure to replicate article-elicited N400 effects. Language, Cognition and Neuroscience, 32(8), 954-965. J. Neural Eng. 11, 035013.

Jacobs, G. D., \& Snyder, D. (1996). Frontal brain asymmetry predicts affective style in men. Behavioral neuroscience, 110(1), 3.

Jiang, X., Bian, G. B., \& Tian, Z. (2019). Removal of artifacts from EEG signals: a review. Sensors, 19(5), 987.

John, L. K., Loewenstein, G., \& Prelec, D. (2012). Measuring the prevalence of questionable research practices with incentives for truth telling. Psychological science, 23(5), 524-532.

Kaida, K., Takahashi, M., Åkerstedt, T., Nakata, A., Otsuka, Y., Haratani, T., \& Fukasawa, K. (2006). Validation of the Karolinska sleepiness scale against performance and EEG variables. Clinical neurophysiology, 117(7), 1574-1581.

Kano, K., Nakamura, M., Matsuoka, T., Iida, H., \& Nakajima, T. (1992). The topographical features of EEGs in patients with affective disorders. Electroencephalography and Clinical Neurophysiology, 83(2), 124-129. 
Keil, A., Debener, S., Gratton, G., Junghöfer, M., Kappenman, E. S., Luck, S. J., ... \& Yee, C. M. (2014). Committee report: publication guidelines and recommendations for studies using electroencephalography and magnetoencephalography. Psychophysiology, 51(1), 121.

Kerr, N. L. (1998). HARKing: Hypothesizing after the results are known. Personality and social psychology review, 2(3), 196-217.

Keune, P. M., Bostanov, V., Kotchoubey, B., \& Hautzinger, M. (2012). Mindfulness versus rumination and behavioral inhibition: A perspective from research on frontal brain asymmetry. Personality and Individual Differences, 53(3), 323-328.

Keune, P. M., Schönenberg, M., Wyckoff, S., Mayer, K., Riemann, S., Hautzinger, M., \& Strehl, U. (2011). Frontal alpha-asymmetry in adults with attention deficit hyperactivity disorder: Replication and specification. Biological psychology, 87(2), 306-310.

Keune, P. M., van der Heiden, L., Várkuti, B., Konicar, L., Veit, R., \& Birbaumer, N. (2012). Prefrontal brain asymmetry and aggression in imprisoned violent offenders. Neuroscience letters, 515(2), 191-195.

Klein, R., Ratliff, K., Vianello, M., Adams Jr, R., Bahník, S., Bernstein, M., ... \& Nosek, B. (2014). Data from investigating variation in replicability: A “many labs" replication project. Journal of Open Psychology Data, 2(1), e4.

Kline, J. P., Blackhart, G. C., Woodward, K. M., Williams, S. R., \& Schwartz, G. E. (2000). Anterior electroencephalographic asymmetry changes in elderly women in response to a pleasant and an unpleasant odor. Biological Psychology, 52(3), 241-250.

Knyazev, G. G., \& Slobodskaya, H. R. (2003). Personality trait of behavioral inhibition is associated with oscillatory systems reciprocal relationships. International journal of psychophysiology, 48(3), 247-261.

Kuper, N., Käckenmester, W., \& Wacker, J. (2019). Resting frontal EEG asymmetry and personality traits: A meta-analysis. European Journal of Personality, 33(2), 154-175.

Lacey, M. F., \& Gable, P. A. (2021). Frontal Asymmetry in an approach-avoidance conflict paradigm. Psychophysiology, 58(5), e13780. 
Lacey, M. F., Neal, L. B., \& Gable, P. A. (2020). Effortful control of motivation, not withdrawal motivation, relates to greater right frontal asymmetry. International Journal of Psychophysiology, 147, 18-25.

Larson, M. J., \& Carbine, K. A. (2017). Sample size calculations in human electrophysiology (EEG and ERP) studies: A systematic review and recommendations for increased rigor. International Journal of Psychophysiology, 111, 33-41.

Larson, M. J., \& Moser, J. S. (2017). Rigor and replication: Toward improved best practices in human electrophysiology research. International Journal of Psychophysiology.

Li, Z., Zhang, L., Zhang, F., Gu, R., Peng, W., \& Hu, L. (2020). Demystifying signal processing techniques to extract resting-state EEG features for psychologists. Brain Science Advances, 6(3), 189-209.

Lin, Z., Chen, M., \& Ma, Y. (2010). The augmented lagrange multiplier method for exact recovery of corrupted low-rank matrices. arXiv preprint arXiv:1009.5055.

Lodder, S. S., \& van Putten, M. J. (2013). Quantification of the adult EEG background pattern. Clinical neurophysiology, 124(2), 228-237.

Luck, S. J. (2014). An introduction to the event-related potential technique (2nd ed.). MIT Press.

Luck, S. J., \& Gaspelin, N. (2017). How to get statistically significant effects in any ERP experiment (and why you shouldn't). Psychophysiology, 54(1), 146-157.

Lykken, D. T. (1991). What's wrong with psychology anyway. Thinking clearly about psychology, 1, 3-39.

du Sautoy, M. (2017), What We Cannot Know: From Consciousness to the Cosmos, the Cutting Edge of Science Explained, 4th Estate.

Mahjoory, K., Nikulin, V. V., Botrel, L., Linkenkaer-Hansen, K., Fato, M. M., \& Haufe, S. (2017). Consistency of EEG source localization and connectivity estimates. Neuroimage, 152, 590-601.

Maltez, J., Hyllienmark, L., Nikulin, V. V., \& Brismar, T. (2004). Time course and variability of power in different frequency bands of EEG during resting conditions. Neurophysiologie Clinique/Clinical Neurophysiology, 34(5), 195-202. 
Mariani, S., Tarokh, L., Djonlagic, I., Cade, B. E., Morrical, M. G., Yaffe, K., ... \& Aeschbach, D. (2018). Evaluation of an automated pipeline for large-scale EEG spectral analysis: the National Sleep Research Resource. Sleep medicine, 47, 126-136.

McEvoy, L. K., Smith, M. E., \& Gevins, A. (2000). Test-retest reliability of cognitive EEG. Clinical Neurophysiology, 111(3), 457-463.

Mechin, N., Gable, P. A., \& Hicks, J. A. (2016). Frontal asymmetry and alcohol cue reactivity: Influence of core personality systems. Psychophysiology, 53(8), 1224-1231.

Miller, A. C., \& Serzan, S. L. (1984). Criteria for identifying a refereed journal. The Journal of Higher Education, 55(6), 673-699.

Mischel, W., Shoda, Y., \& Ayduk, O. (2007). Introduction to personality: Toward an integrative science of the person. John Wiley \& Sons.

Moshontz, H., Campbell, L., Ebersole, C. R., IJzerman, H., Urry, H. L., Forscher, P. S., ... \& Chartier, C. R. (2018). The Psychological Science Accelerator: Advancing psychology through a distributed collaborative network. Advances in Methods and Practices in Psychological Science, 1(4), 501-515.

Muraven, M., \& Baumeister, R. F. (2000). Self-regulation and depletion of limited resources: Does self-control resemble a muscle?. Psychological bulletin, 126(2), 247.

Nakagawa, S. (2004). A farewell to Bonferroni: the problems of low statistical power and publication bias. Behavioral ecology, 15(6), 1044-1045.

Nash, K., Inzlicht, M., \& McGregor, I. (2012). Approach-related left prefrontal EEG asymmetry predicts muted error-related negativity. Biological psychology, 91(1), 96-102.

Neal, L. B., \& Gable, P. A. (2016). Neurophysiological markers of multiple facets of impulsivity. Biological Psychology, 115, 64-68.

Neal, L. B., \& Gable, P. A. (2017). Regulatory control and impulsivity relate to resting frontal activity. Social Cognitive and Affective Neuroscience, 12(9), 1377-1383.

Nieuwland, M. S., Politzer-Ahles, S., Heyselaar, E., Segaert, K., Darley, E., Kazanina, N., ... \& Huettig, F. (2018). Large-scale replication study reveals a limit on probabilistic prediction in language comprehension. ELife, 7, e33468.

Nosek, B. A., Alter, G., Banks, G. C., Borsboom, D., Bowman, S. D., Breckler, S. J., ... \& Yarkoni, (2015). Promoting an open research culture. Science, 348(6242), 1422-1425. 
Nosek, B. A., Spies, J. R., \& Motyl, M. (2012). Scientific utopia: II. Restructuring incentives and practices to promote truth over publishability. Perspectives on Psychological Science, 7(6), 615-631.

O’Donnell, M., Nelson, L. D., Ackermann, E., Aczel, B., Akhtar, A., Aldrovandi, S., ... \& Zrubka, M. (2018). Registered replication report: Dijksterhuis and van Knippenberg (1998). Perspectives on Psychological Science, 13(2), 268-294.

Ocklenburg, S., \& Gunturkun, O. (2017). The lateralized brain: The neuroscience and evolution of hemispheric asymmetries. Academic Press.

Ocklenburg, S., Friedrich, P., Schmitz, J., Schlüter, C., Genc, E., Güntürkün, O., ... \& Grimshaw, G. (2019). Beyond frontal alpha: investigating hemispheric asymmetries over the EEG frequency spectrum as a function of sex and handedness. Laterality: Asymmetries of Body, Brain and Cognition, 24(5), 505-524.

Oldfield, R. C. (1971). The assessment and analysis of handedness: the Edinburgh inventory. Neuropsychologia, 9(1), 97-113.

Open Science Collaboration. (2015). Estimating the reproducibility of psychological science. Science, 349(6251).

Orrison, W. W., Lewine, J., Sanders, J., \& Hartshorne, M. F. (1995). Functional brain imaging. Mosby.

Osborne, J. W. (2013). Normality of residuals is a continuous variable, and does seem to influence the trustworthiness of confidence intervals: A response to, and appreciation of, Williams, Grajales, and Kurkiewicz (2013). Practical Assessment, Research, and Evaluation, 18(1), 12.

Packheiser, J., Schmitz, J., Pan, Y., El Basbasse, Y., Friedrich, P., Güntürkün, O., \& Ocklenburg, S. (2020). Using mobile EEG to investigate alpha and beta asymmetries during hand and foot use. Frontiers in neuroscience, 14, 109.

Papousek, I., \& Schulter, G. (1999). EEG correlates of behavioural laterality: righthandedness. Perceptual and motor skills, 89(2), 403-411.

Pascalis, V. D., Cirillo, G., \& Vecchio, A. (2020). Resting EEG asymmetry markers of multiple facets of the behavioral approach system: A LORETA analysis. Symmetry, 12(11), 1794. 
Patil, P., Peng, R. D., \& Leek, J. T. (2016). What should researchers expect when they replicate studies? A statistical view of replicability in psychological science. Perspectives on Psychological Science, 11(4), 539-544.

Pavlov, Y. G., Adamian, N., Appelhoff, S., Arvaneh, M., Benwell, C. S., Beste, C., ... \& Mushtaq, F. (2021). \# EEGManyLabs: Investigating the replicability of influential EEG experiments. Cortex.

Pedroni, A., Bahreini, A., \& Langer, N. (2019). Automagic: Standardized preprocessing of big EEG data. NeuroImage, 200, 460-473.

Perneger, T. V. (1998). What's wrong with Bonferroni adjustments. Bmj, 316(7139), 12361238.

Petruzzello, S. J., \& Landers, D. M. (1994). State anxiety reduction and exercise: does hemispheric activation reflect such changes?. Medicine \& Science in Sports \& Exercise.

Pion-Tonachini, L., Kreutz-Delgado, K., \& Makeig, S. (2019). ICLabel: An automated electroencephalographic independent component classifier, dataset, and website. NeuroImage, 198, 181-197.

Pizzagalli, D. A., Sherwood, R. J., Henriques, J. B., \& Davidson, R. J. (2005). Frontal brain asymmetry and reward responsiveness: a source-localization study. Psychological science, 16(10), 805-813.

Plank, M. (2014). Independent Component Analysis - demystified!. Retrieved from https://pressrelease.brainproducts.com/independent-component-analysis-demystified/

Poldrack, R. A., Baker, C. I., Durnez, J., Gorgolewski, K. J., Matthews, P. M., Munafò, M. R., ... \& Yarkoni, T. (2017). Scanning the horizon: towards transparent and reproducible neuroimaging research. Nature reviews neuroscience, 18(2), 115-126.

Propper, R. E., Pierce, J., Geisler, M. W., Christman, S. D., \& Bellorado, N. (2012). Asymmetry in resting alpha activity: Effects of handedness. Open Journal of Medical Psychology.1(4), 86-90

Proudfit, G. H., Inzlicht, M., \& Mennin, D. (2013). Anxiety and error monitoring: the importance of motivation and emotion. Frontiers in human neuroscience, 7, 636. 
Pugnaghi, G., Cooper, A., Ettinger, U., \& Corr, P. J. (2018). The psychometric properties of the German language reinforcement sensitivity theory-personality questionnaire (RSTPQ). Journal of individual differences. 39(3), 182-190

Quaedflieg, C. W. E. M., Meyer, T., Smulders, F. T. Y., \& Smeets, T. (2015). The functional role of individual-alpha based frontal asymmetry in stress responding. Biological psychology, 104, 75-81.

Quirin, M., Gruber, T., Kuhl, J., \& Düsing, R. (2013). Is love right? Prefrontal resting brain asymmetry is related to the affiliation motive. Frontiers in human neuroscience, 7, 902.

Rasekhi, J., Mollaei, M. R. K., Bandarabadi, M., Teixeira, C. A., \& Dourado, A. (2013). Preprocessing effects of 22 linear univariate features on the performance of seizure prediction methods. Journal of neuroscience methods, 217(1-2), 9-16.

Reeve, J. (2014). Understanding motivation and emotion. John Wiley \& Sons.

Reuter-Lorenz, P., \& Davidson, R. J. (1981). Differential contributions of the two cerebral hemispheres to the perception of happy and sad faces. Neuropsychologia, 19(4), 609-613.

Reznik, S. J., \& Allen, J. J. (2018). Frontal asymmetry as a mediator and moderator of emotion: An updated review. Psychophysiology, 55(1), e12965.

Robbins, K. A., Touryan, J., Mullen, T., Kothe, C., \& Bigdely-Shamlo, N. (2020). How sensitive are EEG results to preprocessing methods: a benchmarking study. IEEE transactions on neural systems and rehabilitation engineering, 28(5), 1081-1090.

Rodrigues, J., Müller, M., Mühlberger, A., \& Hewig, J. (2018). Mind the movement: Frontal asymmetry stands for behavioral motivation, bilateral frontal activation for behavior. Psychophysiology, 55(1), e12908.

Sabatinelli, D., Bradley, M. M., Fitzsimmons, J. R., \& Lang, P. J. (2005). Parallel amygdala and inferotemporal activation reflect emotional intensity and fear relevance. Neuroimage, 24(4), 1265-1270.

Sandre, A., Banica, I., Riesel, A., Flake, J., Klawohn, J., \& Weinberg, A. (2020). Comparing the effects of different methodological decisions on the error-related negativity and its association with behaviour and gender. International Journal of Psychophysiology, 156, 18-39. 
Schienle, A., Stark, R., Walter, B., Blecker, C., Ott, U., Kirsch, P., ... \& Vaitl, D. (2002). The insula is not specifically involved in disgust processing: an fMRI study. Neuroreport, 13(16), 2023-2026.

Schmeichel, B. J., Harmon-Jones, C., \& Harmon-Jones, E. (2010). Exercising self-control increases approach motivation. Journal of personality and social psychology, 99(1), 162.

Schneider, M., Chau, L., Mohamadpour, M., Stephens, N., Arya, K., \& Grant, A. (2016). EEG asymmetry and BIS/BAS among healthy adolescents. Biological psychology, 120, 142-148.

Schweinsberg, M., Madan, N., Vianello, M., Sommer, S. A., Jordan, J., Tierney, W., ... \& Uhlmann, E. L. (2016). The pipeline project: Pre-publication independent replications of a single laboratory's research pipeline. Journal of Experimental Social Psychology, 66, 5567.

Shackman, A. J., McMenamin, B. W., Maxwell, J. S., Greischar, L. L., \& Davidson, R. J. (2009). Right dorsolateral prefrontal cortical activity and behavioral inhibition. Psychological science, 20(12), 1500-1506.

Shackman, A. J., McMenamin, B. W., Maxwell, J. S., Greischar, L. L., \& Davidson, R. J. (2009). Right dorsolateral prefrontal cortical activity and behavioral inhibition. Psychological science, 20(12), 1500-1506.

Sheorajpanday, R. V., Nagels, G., Weeren, A. J., van Putten, M. J., \& De Deyn, P. P. (2009). Reproducibility and clinical relevance of quantitative EEG parameters in cerebral ischemia: a basic approach. Clinical Neurophysiology, 120(5), 845-855.

Shieh, G. (2010). On the misconception of multicollinearity in detection of moderating effects: Multicollinearity is not always detrimental. Multivariate Behavioral Research, 45(3), 483-507.

Shirk, S. D., McLaren, D. G., Bloomfield, J. S., Powers, A., Duffy, A., Mitchell, M. B., ... \& Atri, A. (2017). Inter-rater reliability of preprocessing EEG data: Impact of subjective artifact removal on associative memory task ERP results. Frontiers in neuroscience, 11, 322.

Shrout, P. E., \& Rodgers, J. L. (2018). Psychology, science, and knowledge construction: Broadening perspectives from the replication crisis. Annual review of psychology, 69, 487510. 
Simmons, J. P., Nelson, L. D., \& Simonsohn, U. (2011). False-positive psychology: Undisclosed flexibility in data collection and analysis allows presenting anything as significant. Psychological science, 22(11), 1359-1366.

Smith, E. E., Reznik, S. J., Stewart, J. L., \& Allen, J. J. (2017). Assessing and conceptualizing frontal EEG asymmetry: An updated primer on recording, processing, analyzing, and interpreting frontal alpha asymmetry. International Journal of Psychophysiology, 111, 98114.

Smits, D. J., \& Kuppens, P. (2005). The relations between anger, coping with anger, and aggression, and the BIS/BAS system. Personality and Individual differences, 39(4), 783793.

Srinivasan, R. (1999). Spatial structure of the human alpha rhythm: global correlation in adults and local correlation in children. Clinical Neurophysiology, 110(8), 1351-1362.

Strobel, A., Beauducel, A., Debener, S., \& Brocke, B. (2001). Eine deutschsprachige version des BIS/BAS-Fragebogens von carver und white. Zeitschrift für Differentielle und diagnostische Psychologie. 22(3), 216-227.

Suarez-Revelo, J., Ochoa-Gomez, J., \& Duque-Grajales, J. (2016, August). Improving testretest reliability of quantitative electroencephalography using different preprocessing approaches. In 2016 38th Annual International Conference of the IEEE Engineering in Medicine and Biology Society (EMBC) (pp. 961-964). IEEE.

Sutton, S. K., \& Davidson, R. J. (1997). Prefrontal brain asymmetry: A biological substrate of the behavioral approach and inhibition systems. Psychological science, 8(3), 204-210.

Szucs, D., \& Ioannidis, J. (2017). When null hypothesis significance testing is unsuitable for research: a reassessment. Frontiers in human neuroscience, 11, 390.

Taylor, P. J. (2010). An introduction to intraclass correlation that resolves some common confusions. Unpublished manuscript, University of Massachusetts, Boston, USA. Retrieved from http://www.faculty.umb.edu/peter_taylor/09b.pdf.

Thibodeau, R., Jorgensen, R. S., \& Kim, S. (2006). Depression, anxiety, and resting frontal EEG asymmetry: a meta-analytic review. Journal of abnormal psychology, 115(4), 715.

Tomarken, A. J., Davidson, R. J., Wheeler, R. E., \& Doss, R. C. (1992). Individual differences in anterior brain asymmetry and fundamental dimensions of emotion. Journal of personality and social psychology, 62(4), 676. 
Tomarken, A. J., Davidson, R. J., Wheeler, R. E., \& Kinney, L. (1992). Psychometric properties of resting anterior EEG asymmetry: Temporal stability and internal consistency. Psychophysiology, 29(5), 576-592.

Towers, D. N., \& Allen, J. J. (2009). A better estimate of the internal consistency reliability of frontal EEG asymmetry scores. Psychophysiology, 46(1), 132-142.

Urigüen, J. A., \& Garcia-Zapirain, B. (2015). EEG artifact removal—state-of-the-art and guidelines. Journal of neural engineering, 12(3), 031001.

Van Der Vinne, N., Vollebregt, M. A., Van Putten, M. J., \& Arns, M. (2017). Frontal alpha asymmetry as a diagnostic marker in depression: Fact or fiction? A metaanalysis. Neuroimage: clinical, 16, 79-87.

VanderWeele, T. J., \& Mathur, M. B. (2019). Some desirable properties of the Bonferroni correction: is the Bonferroni correction really so bad?. American journal of epidemiology, 188(3), 617-618.

Vecchio, A., \& De Pascalis, V. (2020). EEG Resting Asymmetries and Frequency Oscillations in Approach/Avoidance Personality Traits: A Systematic Review. Symmetry, 12(10), 1712.

Vecchio, A., \& De Pascalis, V. (2021). Approach and avoidance personality traits in acute pain and placebo analgesia. Personality and Individual Differences, 169, 109830.

Viola, F. C., Thorne, J., Edmonds, B., Schneider, T., Eichele, T., \& Debener, S. (2009). Semiautomatic identification of independent components representing EEG artifact. Clinical Neurophysiology, 120(5), 868-877.

Wacker, J., Chavanon, M. L., \& Stemmler, G. (2010). Resting EEG signatures of agentic extraversion: New results and meta-analytic integration. Journal of Research in Personality, 44(2), 167-179.

Wacker, J., Chavanon, M. L., Leue, A., \& Stemmler, G. (2008). Is running away right? The behavioral activation-behavioral inhibition model of anterior asymmetry. Emotion, 8(2), 232.

Wacker, J., Chavanon, M.-L., \& Stemmler, G. (2010). Resting EEG signatures of agentic extraversion: New results and meta-analytic integration. Journal of Research in Personality, 44(2), 167-179. 
Wacker, J., Chavanon, M.-L., Leue, A., \& Stemmler, G. (2008). Is running away right? The behavioral activation-behavioral inhibition model of anterior asymmetry. Emotion, 8(2), 232-249.

Wacker, J., Heldmann, M., \& Stemmler, G. (2003). Separating emotion and motivational direction in fear and anger: Effects on frontal asymmetry. Emotion, 3(2), 167-193.

Wheeler, R. E., Davidson, R. J., \& Tomarken, A. J. (1993). Frontal brain asymmetry and emotional reactivity: A biological substrate of affective style. Psychophysiology, 30(1), 8289.

Wiggins, B. J., \& Chrisopherson, C. D. (2019). The replication crisis in psychology: An overview for theoretical and philosophical psychology. Journal of Theoretical and Philosophical Psychology, 39(4), 202.

Winkler, I., Brandl, S., Horn, F., Waldburger, E., Allefeld, C., Tangermann, M., (2014). Robust artifactual independent component classification for BCI practitioners. Journal of neural engineering, 11(3), 035013

Winkler, I., Haufe, S., \& Tangermann, M. (2011). Automatic classification of artifactual ICAcomponents for artifact removal in EEG signals. Behavioral and Brain Functions, 7(1), 115 .

Zhang, L. (2019, July). EEG signals classification using machine learning for the identification and diagnosis of schizophrenia. In 201941 st Annual International Conference of the IEEE Engineering in Medicine and Biology Society (EMBC) (pp. 45214524). IEEE. 


\section{Appendix}

\section{Declaration of Authorship}

Ich versichere, dass ich diese Arbeit selbstständig verfasst und keine andere als die angegebenen Quellen und Hilfsmittel benutzt sowie Zitate kenntlich gemacht habe und dass die pdf-Datei in Inhalt und Wortlaut ausnahmslos der gedruckten Ausfertigung entspricht.

San Salvador, 16.09.2021

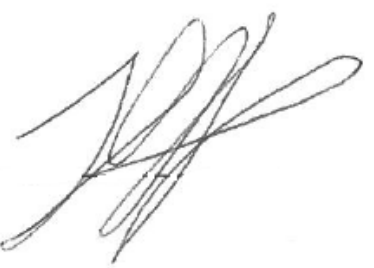

Ort, Datum, Unterschrift 
Supplementary Table 1

Partial Correlations between Personality Scales and Asymmetry Scores after Automated Preprocessing (EC-datasets)

\begin{tabular}{|c|c|c|c|c|c|c|}
\hline Scale & Electrode pair & $\rho$ & & $\mathrm{CI}$ & $\mathrm{S}$ & $p$ \\
\hline BAS & F3F4 & 0.032 & {$[-0.27$} & $0.33]$ & 15692 & $>.999$ \\
\hline BIS & F3F4 & -0.102 & {$[-0.39$} & $0.21]$ & 16731.26 & $>.999$ \\
\hline rBAS & F3F4 & 0.065 & {$[-0.24$,} & $0.36]$ & 14106 & $>.999$ \\
\hline rBIS & F3F4 & 0.276 & {$[-0.02$,} & $0.53]$ & 11736 & 0.631 \\
\hline FFFS & F3F4 & 0.052 & {$[-0.25$,} & $0.34]$ & 15373.97 & $>.999$ \\
\hline BASGDP & F3F4 & 0.372 & {$[0.08$,} & $0.61]$ & 9527.81 & 0.118 \\
\hline BASRI & F3F4 & -0.154 & {$[-0.43$,} & $0.15]$ & 18710.23 & $>.999$ \\
\hline BASRR & F3F4 & -0.031 & {$[-0.33$,} & $0.27]$ & 16718 & $>.999$ \\
\hline BASI & F3F4 & 0.013 & {$[-0.29$,} & $0.31]$ & 16005.97 & $>.999$ \\
\hline BAS & F7F8 & 0.065 & {$[-0.24$,} & $0.36]$ & 15158 & $>.999$ \\
\hline BIS & F7F8 & -0.015 & {$[-0.32$,} & $0.29]$ & 15409.04 & $>.999$ \\
\hline rBAS & F7F8 & 0.014 & {$[-0.29$,} & $0.31]$ & 14966 & $>.999$ \\
\hline rBIS & F7F8 & 0.27 & {$[-0.03$,} & $0.53]$ & 11848 & 0.703 \\
\hline FFFS & F7F8 & 0.017 & {$[-0.28$,} & $0.31]$ & 15943.99 & $>.999$ \\
\hline BASGDP & F7F8 & 0.241 & {$[-0.07$,} & $0.51]$ & 11515.88 & $>.999$ \\
\hline BASRI & F7F8 & -0.311 & {$[-0.56$,} & $-0.01]$ & 21265.47 & 0.351 \\
\hline BASRR & F7F8 & 0.127 & {$[-0.18$,} & $0.41]$ & 14154 & $>.999$ \\
\hline BASI & F7F8 & -0.019 & {$[-0.32$,} & $0.28]$ & 16526.05 & $>.999$ \\
\hline BASG & P3P4 & -0.053 & {$[-0.35$,} & $0.25]$ & 17076 & $>.999$ \\
\hline BIS & P3P4 & -0.129 & {$[-0.41$,} & $0.18]$ & 17142.32 & $>.999$ \\
\hline rBAS & P3P4 & -0.124 & {$[-0.41$,} & $0.18]$ & 17068 & $>.999$ \\
\hline rBIS & P3P4 & -0.137 & {$[-0.41$,} & $0.18]$ & 17142.32 & $>.999$ \\
\hline FFFS & P3P4 & 0.008 & {$[-0.29$,} & $0.31]$ & 16084 & $>.999$ \\
\hline BASGDP & P3P4 & -0.338 & {$[-0.58$,} & $-0.04]$ & 20318.17 & 0.229 \\
\hline BASRI & P3P4 & 0.136 & {$[-0.17$,} & $0.42]$ & 14013.8 & $>.999$ \\
\hline BASRR & P3P4 & -0.203 & {$[-0.47$,} & $0.10]$ & 19510 & $>.999$ \\
\hline BASI & P3P4 & 0.263 & {$[-0.04$,} & $0.52]$ & 11951.34 & 0.775 \\
\hline BAS & P7P8 & 0.285 & {$[-0.02$,} & $0.54]$ & 11600 & 0.552 \\
\hline BIS & P7P8 & -0.018 & {$[-0.32$,} & $0.29]$ & 15456.05 & $>.999$ \\
\hline rBAS & P7P8 & 0.112 & {$[-0.20$,} & $0.40]$ & 13484 & $>.999$ \\
\hline rBIS & P7P8 & -0.277 & {$[-0.53$,} & $0.02]$ & 20706 & 0.624 \\
\hline FFFS & P7P8 & -0.078 & {$[-0.37$,} & $0.23]$ & 17482.04 & $>.999$ \\
\hline BASGDP & P7P8 & -0.07 & {$[-0.36$} & $0.24]$ & 16250.04 & $>.999$ \\
\hline BASRI & P7P8 & 0.268 & {$[-0.03$,} & $0.52]$ & 11870.6 & 0.718 \\
\hline BASRR & P7P8 & 0.012 & {$[-0.29$} & $0.31]$ & 16022 & $>.999$ \\
\hline BASI & P7P8 & 0.149 & {$[-0.16$,} & $0.43]$ & 13806.63 & $>.999$ \\
\hline BAS & $\mathrm{O} 1 \mathrm{O} 2$ & 0.009 & {$[-0.31$,} & $0.29]$ & 16362 & $>.999$ \\
\hline BIS & $\mathrm{O} 1 \mathrm{O} 2$ & -0.081 & {$[-0.37$,} & $0.23]$ & 16405.2 & $>.999$ \\
\hline rBAS & $\mathrm{O} 1 \mathrm{O} 2$ & -0.046 & {$[-0.34$,} & $0.26]$ & 15884 & $>.999$ \\
\hline rBIS & $\mathrm{O} 1 \mathrm{O} 2$ & -0.146 & {$[-0.43$,} & $0.16]$ & 18590 & $>.999$ \\
\hline FFFS & $\mathrm{O} 1 \mathrm{O} 2$ & -0.407 & {$[-0.63$,} & $-0.12]$ & 22821.2 & $0.050^{*}$ \\
\hline BASGDP & $\mathrm{O} 1 \mathrm{O} 2$ & -0.061 & {$[-0.36$,} & $0.25]$ & 16104.03 & $>.999$ \\
\hline BASRI & $\mathrm{O} 1 \mathrm{O} 2$ & 0.07 & {$[-0.23$,} & $0.36]$ & 15083.9 & $>.999$ \\
\hline BASRR & $\mathrm{O} 1 \mathrm{O} 2$ & -0.058 & {$[-0.35$,} & $0.24]$ & 17152 & $>.999$ \\
\hline BASI & $\mathrm{O} 1 \mathrm{O} 2$ & -0.019 & {$[-0.32$,} & $0.28]$ & 16530.05 & $>.999$ \\
\hline
\end{tabular}


Supplementary Table 1 (Continuation)

Correlations between personality scales and asymmetry scores stemming from each electrode pair. The influence of gender, handedness and sleepiness is partialled out. Each coefficient is corrected for multiple comparisons within each partial correlation matrix and not across all combinations of eye-conditions and preprocessing approaches. BAS = Behavioral Activation System, BIS = Behavioral Inhibition System. FFFS = Fight-Flight-Freeze System, CI = confidence interval, GDP = Goal-Drive Persistence, I $=$ Impulsivity, $\mathrm{r}=$ revised, $\mathrm{RI}=$ Reward Interest, $\mathrm{RR}=$ Reward Reactivity $\rho=$ Spearman's rho, $\mathrm{S}=\mathrm{S}$-value, ${ }^{*} p<0.05$ (Bonferroni Correction).

Supplementary Table 2

Partial Correlations between Personality Scales and Asymmetry Scores after Automated Preprocessing (EO-datasets)

\begin{tabular}{|c|c|c|c|c|c|c|}
\hline Scale & Electrode pair & $\rho$ & & & $S$ & $p$ \\
\hline BAS & F3F4 & 0.091 & {$[-0.22$,} & $0.38]$ & 13794 & $>.999$ \\
\hline BIS & $\mathrm{F} 3 \mathrm{~F} 4$ & -0.05 & {$[-0.35$,} & $0.26]$ & 14899.15 & $>.999$ \\
\hline rBAS & F3F4 & -0.261 & {$[-0.52$,} & $0.05]$ & 17898 & 0.866 \\
\hline rBIS & F3F4 & 0.063 & {$[-0.24$,} & $0.36]$ & 14224 & $>.999$ \\
\hline FFFS & $\mathrm{F} 3 \mathrm{~F} 4$ & -0.223 & {$[-0.49$} & $0.08]$ & 18565.11 & $>.999$ \\
\hline BASGDP & F3F4 & -0.13 & {$[-0.42$,} & $0.18]$ & 16038.07 & $>.999$ \\
\hline BASRI & F3F4 & -0.218 & {$[-0.49$,} & $0.09]$ & 18482.22 & $>.999$ \\
\hline BASRR & F3F4 & -0.134 & {$[-0.42$,} & $0.17]$ & 17220 & $>.999$ \\
\hline BASI & $\mathrm{F} 3 \mathrm{~F} 4$ & -0.249 & {$[-0.51$,} & $0.06]$ & 18962.62 & 0.988 \\
\hline BAS & F7F8 & 0.217 & {$[-0.09$,} & $0.49]$ & 11888 & $>.999$ \\
\hline BIS & F7F8 & -0.185 & {$[-0.46$,} & $0.13]$ & 16808.55 & $>.999$ \\
\hline rBAS & F7F8 & 0.006 & {$[-0.30$,} & $0.31]$ & 14118 & $>.999$ \\
\hline rBIS & F7F8 & 0.04 & {$[-0.26$, } & $0.34]$ & 14568 & $>.999$ \\
\hline FFFS & F7F8 & -0.222 & {$[-0.49$,} & $0.09]$ & 18553.11 & $>.999$ \\
\hline BASGDP & F7F8 & 0.166 & {$[-0.15$,} & $0.45]$ & 11828.92 & $>.999$ \\
\hline BASRI & F7F8 & -0.032 & {$[-0.33$,} & $0.27]$ & 15665.03 & $>.999$ \\
\hline BASRR & F7F8 & -0.077 & {$[-0.37$,} & $0.23]$ & 16348 & $>.999$ \\
\hline BASI & F7F8 & 0.048 & {$[-0.26$, } & $0.34]$ & 14447.88 & $>.999$ \\
\hline BASG & P3P4 & 0.013 & {$[-0.29$,} & $0.31]$ & 14982 & $>.999$ \\
\hline BIS & P3P4 & 0.149 & {$[-0.16$, } & $0.43]$ & 12082.55 & $>.999$ \\
\hline rBAS & P3P4 & 0.124 & {$[-0.19$,} & $0.41]$ & 12434 & $>.999$ \\
\hline rBIS & P3P4 & 0.018 & {$[-0.28$,} & $0.32]$ & 14900 & $>.999$ \\
\hline FFFS & P3P4 & 0.155 & {$[-0.15$,} & $0.44]$ & 12826.92 & $>.999$ \\
\hline BASGDP & P3P4 & -0.09 & {$[-0.38$,} & $0.22]$ & 15464.04 & $>.999$ \\
\hline BASRI & P3P4 & 0.159 & {$[-0.15$,} & $0.44]$ & 12772.84 & $>.999$ \\
\hline BASRR & P3P4 & 0.091 & {$[-0.22$,} & $0.38]$ & 13792 & $>.999$ \\
\hline BASI & P3P4 & 0.19 & {$[-0.12$,} & $0.47]$ & 12291.52 & $>.999$ \\
\hline BAS & P7P8 & 0.105 & {$[-0.20$,} & $0.39]$ & 13588 & $>.999$ \\
\hline BIS & P7P8 & -0.031 & {$[-0.33$,} & $0.28]$ & 14636.09 & $>.999$ \\
\hline rBAS & P7P8 & 0.186 & {$[-0.13$,} & $0.46]$ & 11554 & $>.999$ \\
\hline rBIS & P7P8 & -0.193 & {$[-0.47$,} & $0.11]$ & 18116 & $>.999$ \\
\hline FFFS & P7P8 & 0.211 & {$[-0.10$,} & $0.48]$ & 11971.89 & $>.999$ \\
\hline BASGDP & P7P8 & 0.084 & {$[-0.23$,} & $0.38]$ & 13004.96 & $>.999$ \\
\hline BASRI & P7P8 & 0.082 & {$[-0.22$,} & $0.37]$ & 13928.92 & $>.999$ \\
\hline BASRR & P7P8 & 0.115 & {$[-0.19$,} & $0.40]$ & 13436 & $>.999$ \\
\hline BASI & P7P8 & 0.212 & {$[-0.10$,} & $0.48]$ & 11959.47 & $>.999$ \\
\hline
\end{tabular}


Supplementary Table 2 (Continuation)

\begin{tabular}{lllllll} 
BAS & $\mathrm{O} 1 \mathrm{O} 2$ & -0.035 & {$[-0.33$,} & $0.27]$ & 15718 & $>.999$ \\
$\mathrm{BIS}$ & $\mathrm{O} 1 \mathrm{O} 2$ & 0.059 & {$[-0.25$,} & $0.36]$ & 13348.82 & $>.999$ \\
rBAS & $\mathrm{O} 1 \mathrm{O} 2$ & 0.02 & {$[-0.29$,} & $0.32]$ & 13904 & $>.999$ \\
rBIS & $\mathrm{O} 1 \mathrm{O} 2$ & 0.127 & {$[-0.18$,} & $0.41]$ & 13254 & $>.999$ \\
FFFS & $\mathrm{O} 1 \mathrm{O} 2$ & -0.244 & {$[-0.51$,} & $0.06]$ & 18881.12 & $>.999$ \\
BASGDP & $\mathrm{O} 1 \mathrm{O} 2$ & 0.018 & {$[-0.29$,} & $0.32]$ & 13927.99 & $>.999$ \\
BASRI & $\mathrm{O} 1 \mathrm{O} 2$ & -0.083 & {$[-0.38$,} & $0.22]$ & 16437.08 & $>.999$ \\
BASRR & $\mathrm{O} 1 \mathrm{O} 2$ & -0.007 & {$[-0.31$,} & $0.30]$ & 15286 & $>.999$ \\
BASI & $\mathrm{O} 1 \mathrm{O} 2$ & 0.017 & {$[-0.29$,} & $0.32]$ & 14916.96 & $>.999$ \\
\hline
\end{tabular}

Correlations between personality scales and asymmetry scores stemming from each electrode pair. The influence of gender, handedness and sleepiness is partialled out. Each coefficient is corrected for multiple comparisons within each partial correlation matrix and not across all combinations of eye-conditions and preprocessing approaches. BAS = Behavioral Activation System, BIS = Behavioral Inhibition System. FFFS = Fight-Flight-Freeze System, CI = confidence interval, GDP = Goal-Drive Persistence, I $=$ Impulsivity, $\mathrm{r}=$ revised, $\mathrm{RI}=$ Reward Interest, $\mathrm{RR}=$ Reward Reactivity $\rho=$ Spearman's rho, $\mathrm{S}=\mathrm{S}$-value, ${ }^{*} p<0.05$ (Bonferroni Correction).

Supplementary Table 3

Partial Correlations between Personality Scales and Asymmetry Scores after Manual Preprocessing (EC-datasets)

\begin{tabular}{lllllll}
\hline Scale & Electrode pair & $\rho$ & \multicolumn{2}{c}{$95 \%$ CI } & $\mathrm{S}$ & $p$ \\
\hline BAS & F3F4 & -0.05 & {$[-0.35$,} & $0.26]$ & 13904 & $>.999$ \\
BIS & F3F4 & -0.008 & {$[-0.32$,} & $0.30]$ & 12442.01 & $>.999$ \\
rBAS & F3F4 & -0.377 & {$[-0.62$,} & $-0.07]$ & 16998 & 0.138 \\
rBIS & F3F4 & 0.279 & {$[-0.03$,} & $0.54]$ & 9550 & 0.701 \\
FFFS & F3F4 & 0.106 & {$[-0.21$,} & $0.40]$ & 11842 & $>.999$ \\
BASGDP & F3F4 & -0.106 & {$[-0.40$,} & $0.21]$ & 13644.05 & $>.999$ \\
BASRI & F3F4 & -0.296 & {$[-0.55$,} & $0.01]$ & 17161.44 & 0.541 \\
BASRR & F3F4 & -0.248 & {$[-0.52$,} & $0.07]$ & 16522 & $>.999$ \\
BASI & F3F4 & -0.289 & {$[-0.55$,} & $0.02]$ & 17068.72 & 0.604 \\
BAS & F7F8 & 0.084 & {$[-0.23$,} & $0.38]$ & 12136 & $>.999$ \\
BIS & F7F8 & 0.174 & {$[-0.15$,} & $0.46]$ & 10196.74 & $>.999$ \\
rBAS & F7F8 & -0.035 & {$[-0.34$,} & $0.28]$ & 12772 & $>.999$ \\
rBIS & F7F8 & 0.189 & {$[-0.13$,} & $0.47]$ & 10740 & $>.999$ \\
FFFS & F7F8 & -0.031 & {$[-0.34$,} & $0.28]$ & 13654 & $>.999$ \\
BASGDP & F7F8 & 0.103 & {$[-0.22$,} & $0.40]$ & 11068.95 & $>.999$ \\
BASRI & F7F8 & -0.274 & {$[-0.54$,} & $0.04]$ & 16874.41 & 0.753 \\
BASRR & F7F8 & 0.052 & {$[-0.26$,} & $0.36]$ & 12552 & $>.999$ \\
BASI & F7F8 & -0.034 & {$[-0.34$,} & $0.28]$ & 13698.09 & $>.999$ \\
BASG & P3P4 & -0.089 & {$[-0.39$,} & $0.23]$ & 14426 & $>.999$ \\
BIS & P3P4 & 0.001 & {$[-0.31$,} & $0.31]$ & 12328 & $>.999$ \\
rBAS & P3P4 & -0.105 & {$[-0.40$,} & $0.21]$ & 13632 & $>.999$ \\
rBIS & P3P4 & 0.034 & {$[-0.28$,} & $0.34]$ & 12800 & $>.999$ \\
FFFS & P3P4 & 0.161 & {$[-0.16$,} & $0.45]$ & 11116 & $>.999$ \\
BASGDP & P3P4 & -0.366 & {$[-0.61$,} & $-0.06]$ & 16855.18 & 0.172 \\
BASRI & P3P4 & 0.082 & {$[-0.23$,} & $0.38]$ & 12163.88 & $>.999$ \\
BASRR & P3P4 & -0.161 & {$[-0.45$,} & $0.16]$ & 15380 & $>.999$ \\
BASI & P3P4 & 0.355 & {$[0.05$,} & $0.60]$ & 8547.11 & 0.196
\end{tabular}


Supplementary Table 3 (Continuation)

\begin{tabular}{lllllll} 
BAS & P7P8 & 0.127 & {$[-0.19$,} & $0.42]$ & 11556 & $>.999$ \\
BIS & P7P8 & -0.08 & {$[-0.38$,} & $0.24]$ & 13334.12 & $>.999$ \\
rBAS & P7P8 & 0.17 & {$[-0.15$,} & $0.46]$ & 10244 & $>.999$ \\
rBIS & P7P8 & -0.267 & {$[-0.53$,} & $0.05]$ & 16776 & 0.839 \\
FFFS & P7P8 & 0.06 & {$[-0.25$,} & $0.36]$ & 12454 & $>.999$ \\
BASGDP & P7P8 & 0.006 & {$[-0.31$,} & $0.32]$ & 12261 & $>.999$ \\
BASRI & P7P8 & 0.231 & {$[-0.08$,} & $0.50]$ & 10182.65 & $>.999$ \\
BASRR & P7P8 & 0.127 & {$[-0.19$,} & $0.42]$ & 11558 & $>.999$ \\
BASI & P7P8 & 0.294 & {$[-0.02$,} & $0.55]$ & 9346.26 & 0.554 \\
BAS & O1O2 & -0.066 & {$[-0.37$,} & $0.25]$ & 14122 & $>.999$ \\
BIS & O1O2 & -0.049 & {$[-0.36$,} & $0.27]$ & 12948.07 & $>.999$ \\
rBAS & O1O2 & 0.036 & {$[-0.28$,} & $0.34]$ & 11902 & $>.999$ \\
rBIS & O1O2 & 0.038 & {$[-0.27$,} & $0.34]$ & 12744 & $>.999$ \\
FFFS & O1O2 & -0.162 & {$[-0.45$,} & $0.15]$ & 15386 & $>.999$ \\
BASGDP & O1O2 & 0.009 & {$[-0.30$,} & $0.32]$ & 12234 & $>.999$ \\
BASRI & O1O2 & -0.049 & {$[-0.35$,} & $0.26]$ & 13896.07 & $>.999$ \\
BASRR & O1O2 & 0.094 & {$[-0.22$,} & $0.39]$ & 12002 & $>.999$ \\
BASI & O1O2 & 0.078 & {$[-0.24$,} & $0.38]$ & 12214.81 & $>.999$ \\
\hline
\end{tabular}

Correlations between personality scales and asymmetry scores stemming from each electrode pair. The influence of gender, handedness and sleepiness is partialled out. Each coefficient is corrected for multiple comparisons within each partial correlation matrix and not across all combinations of eye-conditions and preprocessing approaches. BAS $=$ Behavioral Activation System, BIS = Behavioral Inhibition System. FFFS = Fight-Flight-Freeze System, CI = confidence interval, GDP = Goal-Drive Persistence, I $=$ Impulsivity, $\mathrm{r}=$ revised, RI $=$ Reward Interest, $\mathrm{RR}=$ Reward Reactivity $\rho=$ Spearman's rho, $\mathrm{S}=\mathrm{S}$-value, ${ }^{*} p<0.05$ (Bonferroni Correction).

Supplementary Table 4 Partial Correlations between Personality Scales and Asymmetry Scores after Manual Preprocessing (EO-datasets)

\begin{tabular}{lllllll}
\hline Scale & Electrode pair & $\rho$ & \multicolumn{2}{c}{$95 \%$ CI } & S & $p$ \\
\hline BAS & F3F4 & 0.261 & {$[-0.06$,} & $0.53]$ & 9124 & 0.954 \\
BIS & F3F4 & -0.098 & {$[-0.40$,} & $0.22]$ & 12608.15 & $>.999$ \\
rBAS & F3F4 & -0.098 & {$[-0.40$,} & $0.22]$ & 12608.15 & $>.999$ \\
rBIS & F3F4 & 0.188 & {$[-0.14$,} & $0.48]$ & 9318 & $>.999$ \\
FFFS & F3F4 & 0.059 & {$[-0.26$,} & $0.36]$ & 11614 & $>.999$ \\
BASGDP & F3F4 & -0.102 & {$[-0.40$,} & $0.22]$ & 13595.05 & $>.999$ \\
BASRI & F3F4 & 0.036 & {$[-0.28$,} & $0.35]$ & 11063.98 & $>.999$ \\
BASRR & F3F4 & 0.241 & {$[-0.08$,} & $0.51]$ & 9369.76 & $>.999$ \\
BASI & F3F4 & 0.068 & {$[-0.25$,} & $0.37]$ & 11498 & $>.999$ \\
BAS & F7F8 & 0.12 & {$[-0.20$,} & $0.42]$ & 10855.7 & $>.999$ \\
BIS & F7F8 & 0.076 & {$[-0.24$,} & $0.38]$ & 11400 & $>.999$ \\
rBAS & F7F8 & -0.142 & {$[-0.44$,} & $0.18]$ & 13110.21 & $>.999$ \\
rBIS & F7F8 & -0.308 & {$[-0.57$,} & $0.01]$ & 15018 & 0.5 \\
FFFS & F7F8 & 0.009 & {$[-0.30$,} & $0.32]$ & 12226 & $>.999$ \\
BASGDP & F7F8 & -0.166 & {$[-0.45$,} & $0.15]$ & 14391.08 & $>.999$ \\
BASRI & F7F8 & -0.127 & {$[-0.43$,} & $0.20]$ & 12938.06 & $>.999$ \\
BASRR & F7F8 & -0.194 & {$[-0.48$,} & $0.13]$ & 14729.19 & $>.999$ \\
BASI & F7F8 & -0.215 & {$[-0.49$,} & $0.10]$ & 14998 & $>.999$
\end{tabular}


Supplementary Table 4 (Continuation)

\begin{tabular}{lllllll} 
BASG & P3P4 & -0.233 & {$[-0.51$,} & $0.09]$ & 15212.58 & $>.999$ \\
BIS & P3P4 & -0.475 & {$[-0.69$,} & $-0.19]$ & 18200 & $0.015^{*}$ \\
rBAS & P3P4 & -0.008 & {$[-0.32$,} & $0.31]$ & 11577.01 & $>.999$ \\
rBIS & P3P4 & -0.211 & {$[-0.49$,} & $0.11]$ & 13900 & $>.999$ \\
FFFS & P3P4 & 0.237 & {$[-0.08$,} & $0.51]$ & 9422 & $>.999$ \\
BASGDP & P3P4 & -0.145 & {$[-0.44$,} & $0.17]$ & 14134.07 & $>.999$ \\
BASRI & P3P4 & -0.356 & {$[-0.60$,} & $-0.05]$ & 15571.18 & 0.222 \\
BASRR & P3P4 & -0.126 & {$[-0.42$,} & $0.19]$ & 13898.13 & $>.999$ \\
BASI & P3P4 & -0.287 & {$[-0.55$,} & $0.03]$ & 15884 & 0.653 \\
BAS & P7P8 & 0.275 & {$[-0.04$,} & $0.54]$ & 8942.31 & 0.775 \\
BIS & P7P8 & -0.116 & {$[-0.41$,} & $0.20]$ & 13768 & $>.999$ \\
rBAS & P7P8 & 0.19 & {$[-0.13$,} & $0.48]$ & 9295.71 & $>.999$ \\
rBIS & P7P8 & 0.132 & {$[-0.19$,} & $0.43]$ & 9964 & $>.999$ \\
FFFS & P7P8 & 0.113 & {$[-0.21$,} & $0.41]$ & 10948 & $>.999$ \\
BASGDP & P7P8 & 0.153 & {$[-0.17$,} & $0.44]$ & 10447.92 & $>.999$ \\
BASRI & P7P8 & -0.139 & {$[-0.44$,} & $0.19]$ & 13071.07 & $>.999$ \\
BASRR & P7P8 & 0.172 & {$[-0.15$,} & $0.46]$ & 10223.83 & $>.999$ \\
BASI & P7P8 & 0.169 & {$[-0.15$,} & $0.46]$ & 10254 & $>.999$ \\
BAS & O1O2 & 0.224 & {$[-0.09$,} & $0.50]$ & 9573.44 & $>.999$ \\
BIS & O1O2 & -0.16 & {$[-0.45$,} & $0.16]$ & 14314 & $>.999$ \\
rBAS & O1O2 & -0.447 & {$[-0.67$,} & $-0.15]$ & 16609.67 & $0.034 *$ \\
rBIS & O1O2 & -0.13 & {$[-0.43$,} & $0.19]$ & 12978 & $>.999$ \\
FFFS & O1O2 & -0.138 & {$[-0.43$,} & $0.18]$ & 14040 & $>.999$ \\
BASGDP & O1O2 & 0.002 & {$[-0.31$,} & $0.31]$ & 12317 & $>.999$ \\
BASRI & O1O2 & -0.047 & {$[-0.36$,} & $0.27]$ & 12021.02 & $>.999$ \\
BASRR & O1O2 & 0.111 & {$[-0.21$,} & $0.41]$ & 10975.89 & $>.999$ \\
BASI & O1O2 & -0.119 & {$[-0.42$,} & $0.20]$ & 13814 & $>.999$ \\
\hline ConIa & bew &
\end{tabular}

Correlations between personality scales and asymmetry scores stemming from each electrode pair. The influence of gender, handedness and sleepiness is partialled out. Each coefficient is corrected for multiple comparisons within each partial correlation matrix and not across all combinations of eye-conditions and preprocessing approaches. BAS = Behavioral Activation System, BIS = Behavioral Inhibition System. FFFS = Fight-Flight-Freeze System, CI = confidence interval, GDP = Goal-Drive Persistence, I $=$ Impulsivity, $\mathrm{r}=$ revised, $\mathrm{RI}=$ Reward Interest, $\mathrm{RR}=$ Reward Reactivity $\rho=$ Spearman's rho, $\mathrm{S}=\mathrm{S}$-value, $* p<0.05$ (Bonferroni Correction). 

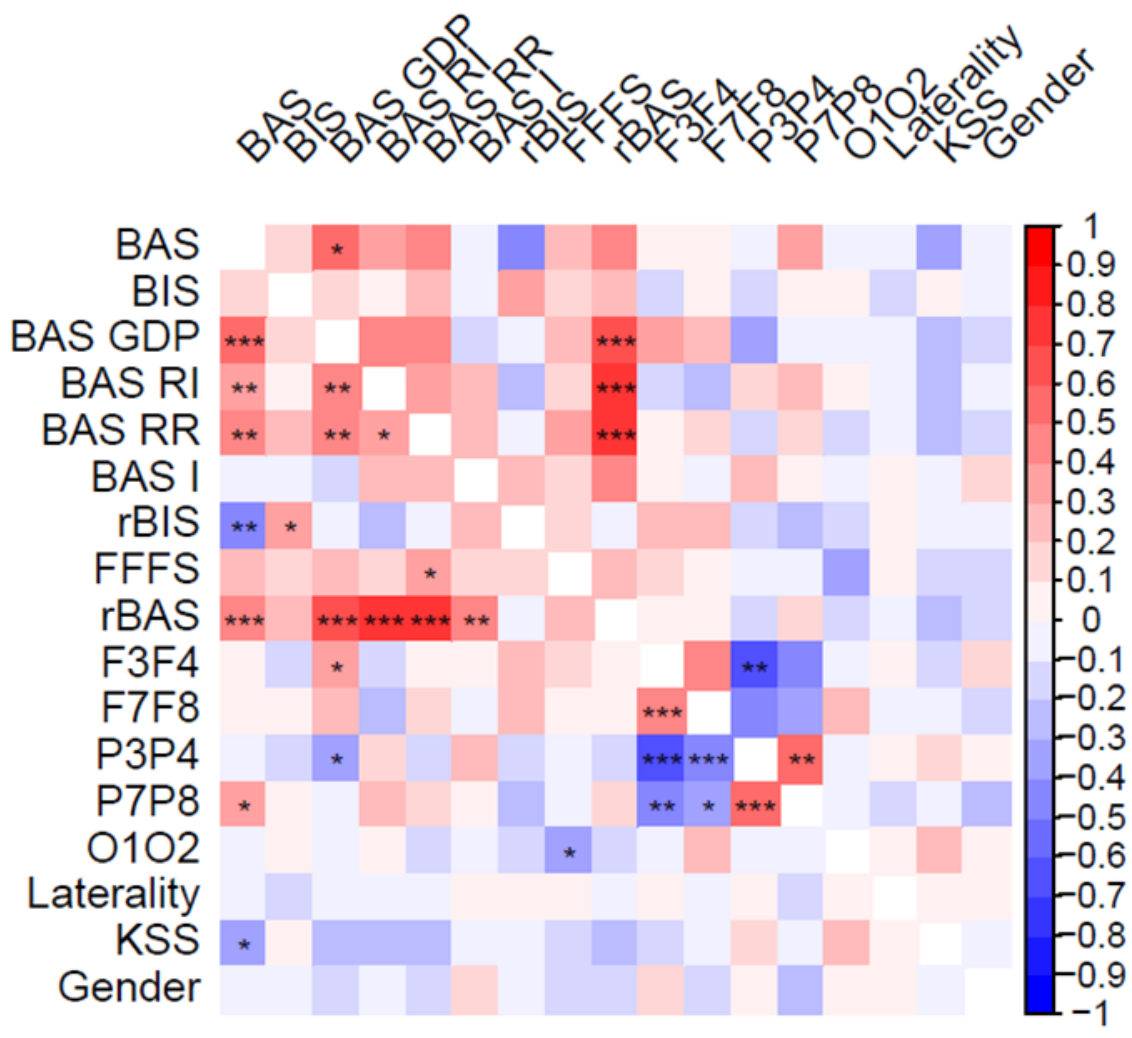

Supp.Fig.1. Correlation Heatmap. All personality scales, covariates and asymmetry scores obtained after automated preprocessing of EC-datasets are included. Positive and negative correlations (Spearman's rho) are coded red and blue, respectively. Coefficients at the diagonal are omitted. $p$-values above the diagonal are Bonferroni-corrected for multiple comparisons. Handedness is represented by "Laterality". Laterality was operationalized by computing the LQ. BAS = Behavioral Activation System, BIS = Behavioral Inhibition System, FFFS $=$ Fight-Flight-Freeze-System, GDP = Goal-Drive Persistence, KSS = Karolinska Sleepiness Scale, RI = Reward Interest, RR = Reward Reactivity, I = Impulsivity, $\mathrm{r}=$ revised $* p<0.05, * * p<0.01$, *** $p<0.001$. 

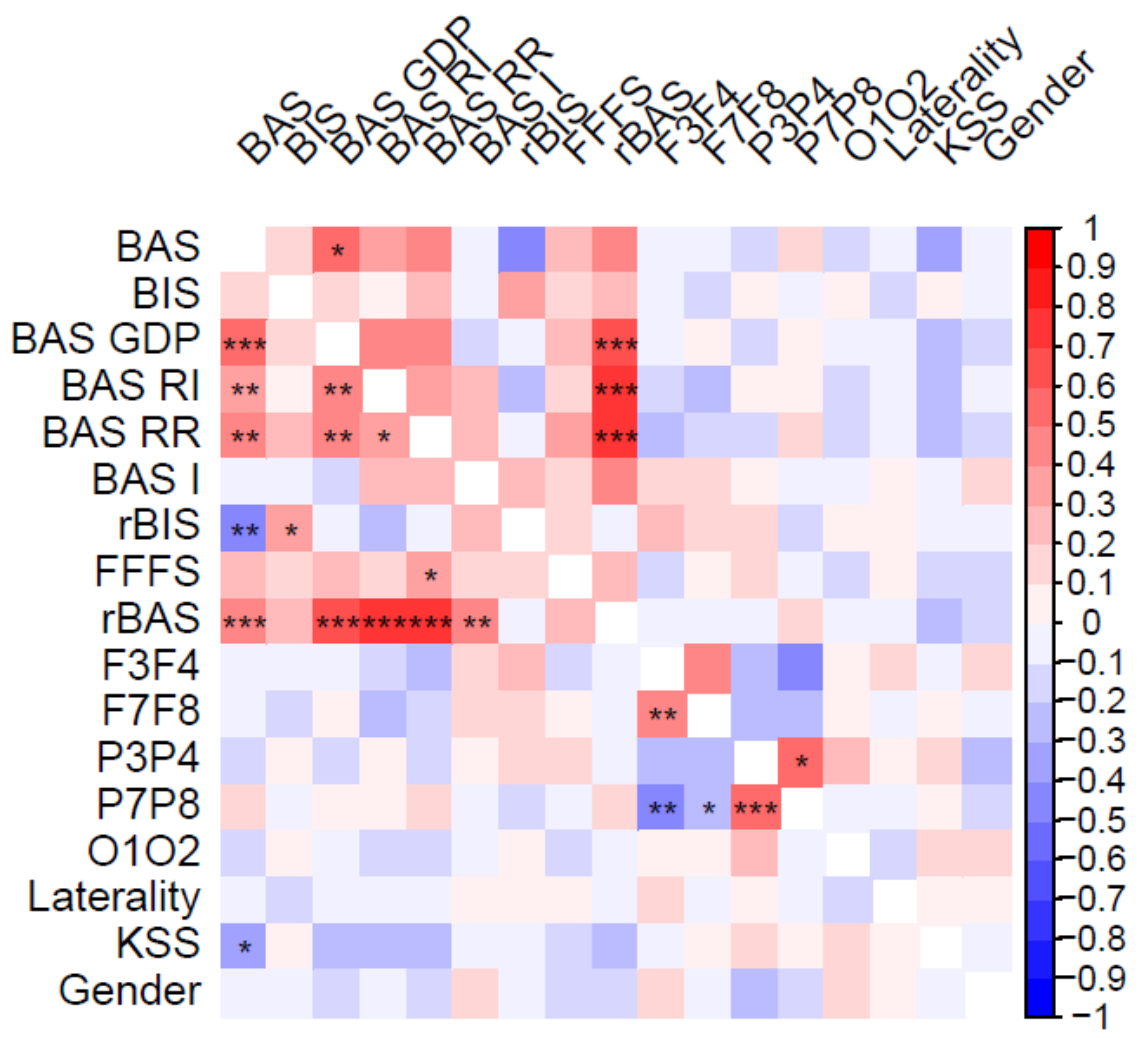

Supp.Fig.2. Correlation Heatmap. All personality scales, covariates and asymmetry scores obtained after automated preprocessing of EO-datasets are included. Positive and negative correlations (Spearman's rho) are coded red and blue, respectively. Coefficients at the diagonal are omitted. $p$-values above the diagonal are Bonferroni-corrected for multiple comparisons. Handedness is represented by "Laterality". Laterality was operationalized by computing the LQ. BAS = Behavioral Activation System, BIS = Behavioral Inhibition System, FFFS = Fight-Flight-Freeze-System, GDP = Goal-Drive Persistence, KSS = Karolinska Sleepiness Scale, RI = Reward Interest, RR = Reward Reactivity, I = Impulsivity, $\mathrm{r}=$ revised $* p<0.05, * * p<0.01, * * * p<0.001$. 

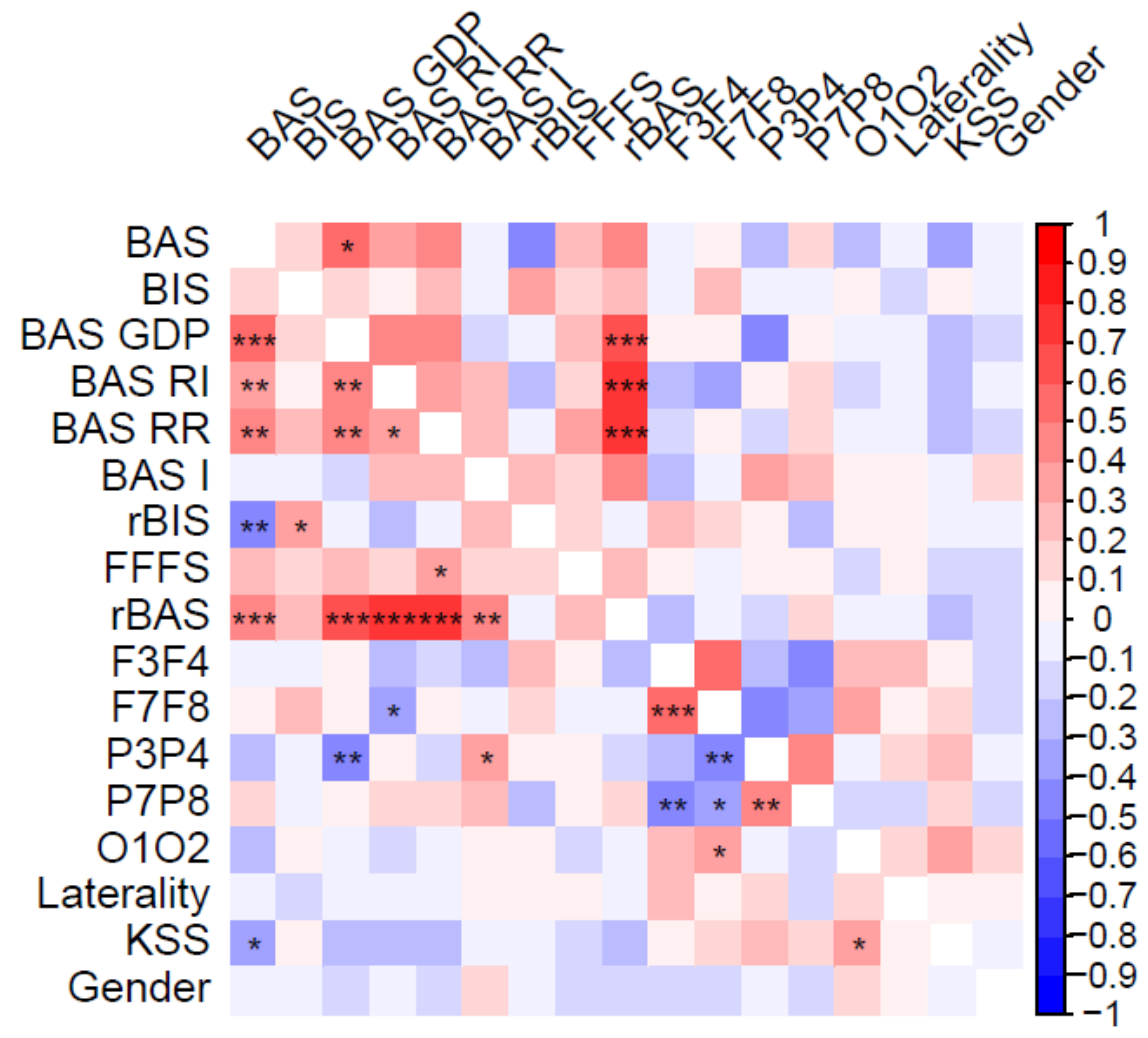

Supp.Fig.3. Correlation Heatmap. All personality scales, covariates and asymmetry scores obtained after manual preprocessing of EC-datasets are included. Positive and negative correlations (Spearman's rho) are coded red and blue, respectively. Coefficients at the diagonal are omitted. $p$-values above the diagonal are Bonferroni-corrected for multiple comparisons. Handedness is represented by "Laterality". Laterality was operationalized by computing the LQ. BAS = Behavioral Activation System, BIS = Behavioral Inhibition System, FFFS = Fight-Flight-Freeze-System, GDP = Goal-Drive Persistence, KSS = Karolinska Sleepiness Scale, RI = Reward Interest, RR = Reward Reactivity, I = Impulsivity, $\mathrm{r}=$ revised $* p<0.05, * * p<0.01, * * * p<0.001$. 

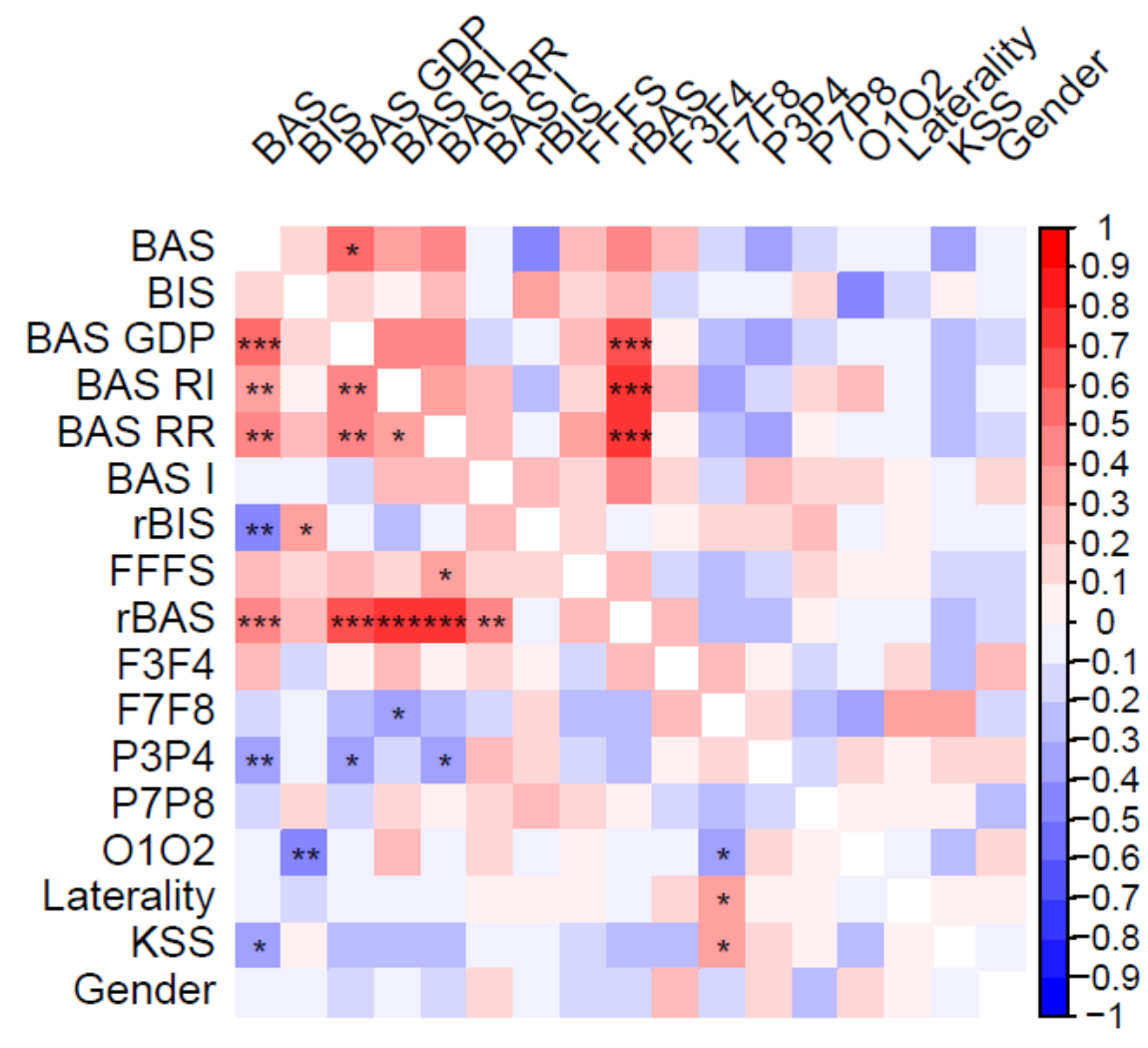

Supp.Fig.4. Correlation Heatmap. All personality scales, covariates and asymmetry scores obtained after manual preprocessing of EO-datasets are included. Positive and negative correlations (Spearman's rho) are coded red and blue, respectively. Coefficients at the diagonal are omitted. $p$-values above the diagonal are Bonferroni-corrected for multiple comparisons. Handedness is represented by "Laterality". Laterality was operationalized by computing the LQ. BAS = Behavioral Activation System, BIS = Behavioral Inhibition System, FFFS $=$ Fight-Flight-Freeze-System, GDP = Goal-Drive Persistence, KSS = Karolinska Sleepiness Scale, RI = Reward Interest, RR = Reward Reactivity, I = Impulsivity, $\mathrm{r}=$ revised $* p<0.05, * * p<0.01$, *** $p<0.001$. 